\title{
Learning from Reliable and Unreliable Speakers
}

\section{Early Development and Underlying Mechanisms}

\author{
Dissertation \\ zur Erlangung des mathematisch-naturwissenschaftlichen Doktorgrades \\ „Doctor rerum naturalium" \\ der Georg-August-Universität Göttingen
}

im Promotionsstudiengang Behavior and Cognition (BeCog) der Georg-August University School of Science (GAUSS)

\author{
vorgelegt von \\ Benjamin Schmid \\ aus Augsburg
}

Göttingen, 2018 


\section{Betreuungsausschuss}

Dr. Tanya Behne, Abt. Entwicklungspsychologie, Georg-EliasMüller-Institut für Psychologie (GEMI), Universität Göttingen

Prof. Dr. Nivedita Mani, Abt. Psychologie der Sprache, GEMI, Universität Göttingen

Prof. Dr. Julia Fischer, Abt. Kognitive Ethologie, Deutsches Primatenzentrum Göttingen (DPZ)

Mitglieder der Prüfungskommission

Referentin: Dr. Tanya Behne, Abt. Entwicklungspsychologie, GEMI, Universität Göttingen

Korreferentin: Prof. Dr. Nivedita Mani, Abt. Psychologie der Sprache, GEMI, Universität Göttingen

Weitere Mitglieder der Prüfungskommission:

Prof. Dr. Julia Fischer, Abt. Kognitive Ethologie, DPZ

Prof. Dr. Hannes Rakoczy, Abt. Entwicklungspsychologie, GEMI, Universität Göttingen

Prof. Dr. Michael Waldmann, Abt. Kognitionswissenschaft und Entscheidungspsychologie GEMI, Universität Göttingen

Prof. Dr. Annekathrin Schacht, Abt. Affektive Neurowissenschaft und Psychophysiologie, GEMI, Universität Göttingen

Tag der mündlichen Prüfung: 5. Dezember 2018 


\begin{abstract}
As a way to bypass the need for the many iterations of individual experiences required for trial and error learning, humans and several non-human animal species have evolved a capacity to rely on information provided by others. Non-human animals typically learn from conspecifics through stimulus enhancement or emulation learning, using the same tools a conspecific used to obtain the same goal or trying to replicate the outcome of certain behavior. Human children on the other hand do not just focus on the outcome when learning from others. They pay a lot of attention to the teacher and the teaching process, draw inferences from ostensive cues, and try to replicate actions faithfully with a focus not just on what is demonstrated but also how. This high-fidelity in reliance on socially conveyed information gives rise to human cumulative culture and appears to be unique in the animal kingdom. However, it can also lead to biases, as can be seen in children's tendency to overimitate demonstrated behavior, even if it is not instrumental in obtaining a certain result. In the domain of testimony, we also find these apparent artefacts of children's credulity in their reliance on others. Children will sometimes reproduce testimony by an adult even if the reliability of this testimony is questionable. However, children are not always this gullible. In fact, much research has demonstrated preschoolers' selective trust in some sources over others, based on several distinct features of these sources. An area that has received considerably coverage in recent developmental research is children's inferences from past verbal utterances as an estimate of future reliability of the same source of testimony.
\end{abstract}


While the phenomenon is well documented, less is known about its cognitive underpinnings and early development. How do children generally treat testimony from an unreliable informant? Do they always encode testimony from reliable and unreliable sources differently? Are differences in learning from reliable and unreliable speakers influenced by the extent to which these speakers cohere or contradict each other? And to what extent do toddlers rely on the same strategies as preschoolers? Or do they simply learn less in unusual or confusing situations? In this thesis, I try to discern under what circumstances and to what degree children will continue to rely on testimony from unreliable sources, what mechanisms underlie their selective trust in testimony and examine the early development of this ability. To address these issues, I conducted several experiments with 2- and 5-yearold children examining their trust in testimony using both eye-tracking and interactive measures.

Whereas 5-year-olds demonstrated selective trust across a range of tasks and measures, 2-year-olds showed no evidence of selectively learning novel labels from the more reliable source. If the two speakers provided conflicting information, toddlers performed at chance level, rather than selectively endorsing the information by the more reliable source. However, in a follow-up study that presented the same word-learning demands, but not necessarily the need to consider the speakers' respective reliability, 2-year-olds showed successful learning of novel labels. Thus, toddlers seemed to have struggled with making personspecific attribution of reliability rather than with the tasks' word learning demands. 5-year-olds on the other hand are able to make person-specific inferences about the 
reliability of an informant and adjust their reliance according to the pragmatics of the situation. It appears that children are willing to learn even from a previously unreliable speaker, given that the information offered by her is not contradicted by a more reliable source. However, they do not simply discount accuracy information. Rather, in case contradictory testimony from a less unreliable source surfaces later on, 5-year-olds can still adjust their reliance and discount information from a demonstrably unreliable source.

Taken together, I find that children do not generally encode information from reliable and unreliable sources differently, but they are also not gullible. My findings suggest that children's selective learning of novel labels is not simply based on inattention towards the information provided by unreliable source, but rather on the selective encoding and consolidation of the semantic information provided by a more reliable source. At least at five years of age, human children make rational inferences about the reliability of sources. Even if they initially rely on questionable testimony with apparent credulity, these inferences let children adjust their reliance before deciding whether to reproduce such testimony. Infants likely rely on less demanding strategies than preschoolers to adjust their reliance on testimony from others, as they are not yet able to entertain person-specific inferences. In conclusion, children's trust is testimony appears to be the result of opposing forces in form of attribution of reliability to particular sources of testimony on one hand and a credulity in reliance on socially conveyed information on the other hand. 


\section{Table of Contents}

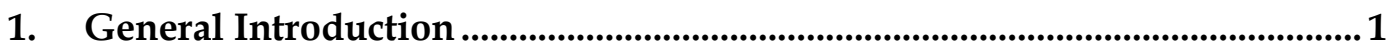

1.1. Origins of Social Learning ................................................................

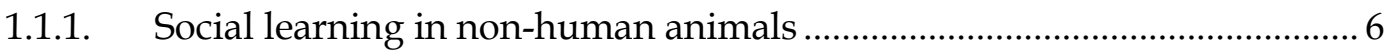

1.1.2. Differences to human social learning........................................................ 9

1.2. Origins of Selective Social Learning............................................................ 11

1.2.1. Selective social learning in animals: When to copy .................................. 13

1.2.2. Selective social learning in animals: Whom to copy ................................ 14

1.2.3. Origins of selective trust in testimony ....................................................... 15

1.3. Early Development of Selective Trust in Testimony................................ 17

1.3.1. Reliability attribution in preschoolers ...................................................... 19

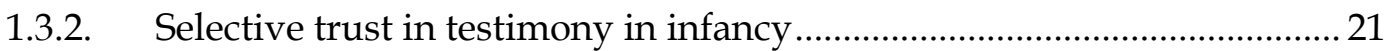

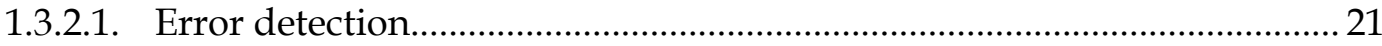

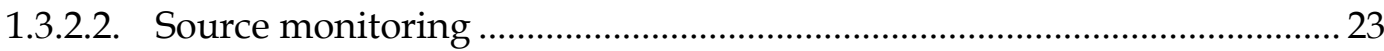

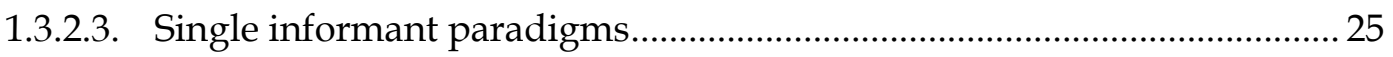

1.4. Underlying Mechanisms of Selective Trust in Testimony .................... 27

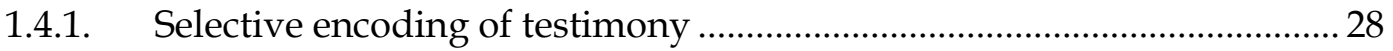

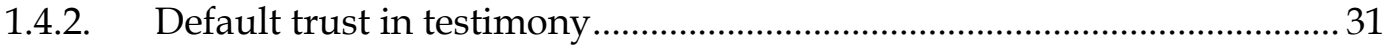

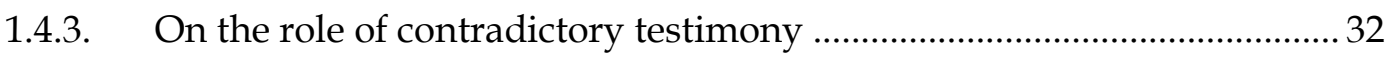

1.4.4. Contradictory testimony as trigger of children's selectivity?................... 35 
2. Study Set 1: Underlying Mechanisms of Selective Trust in Testimony ..... 39

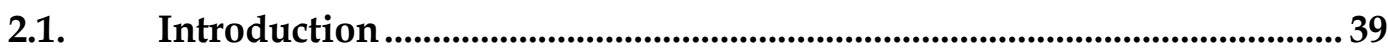

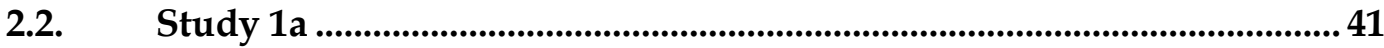

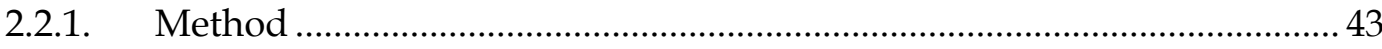

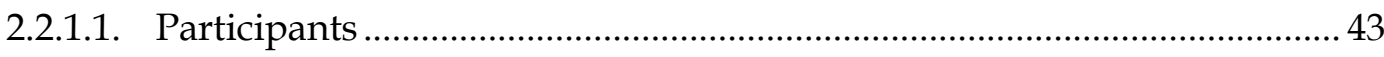

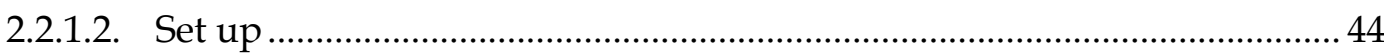

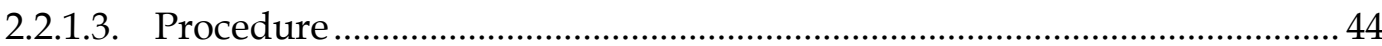

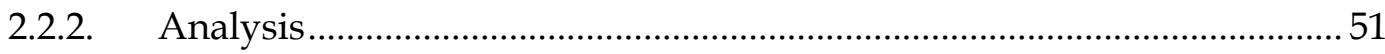

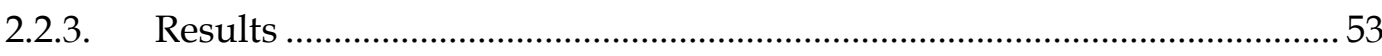

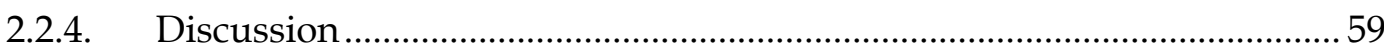

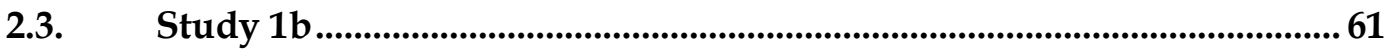

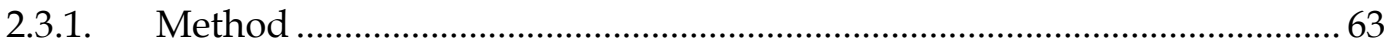

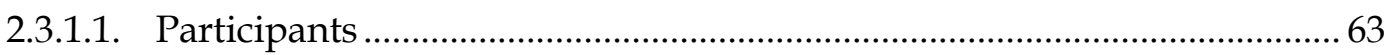

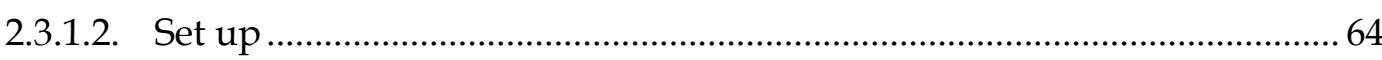

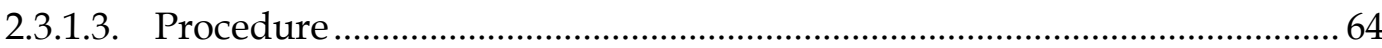

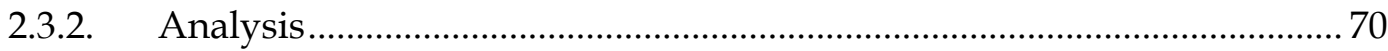

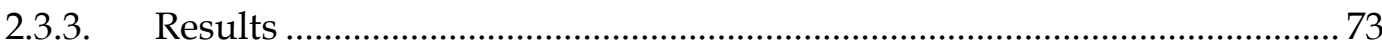

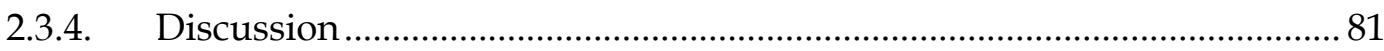

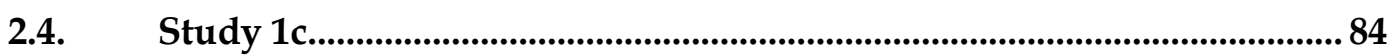

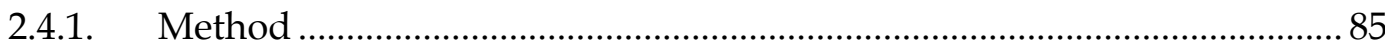

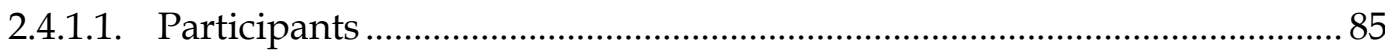

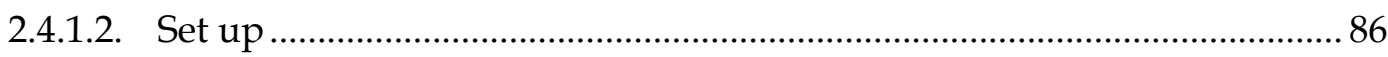

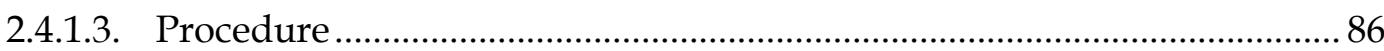

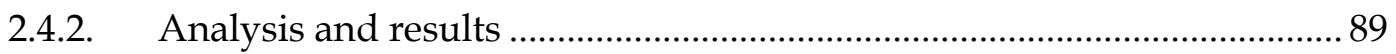

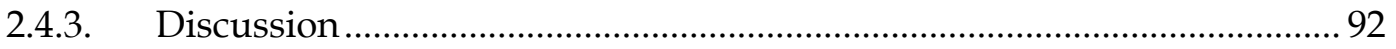


3. Study Set 2: Early Development of Selective Trust in Testimony................ 94

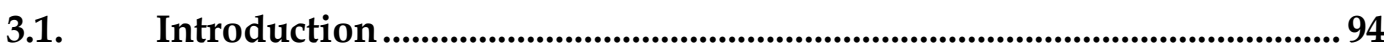

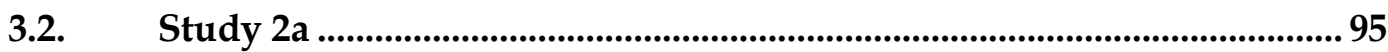

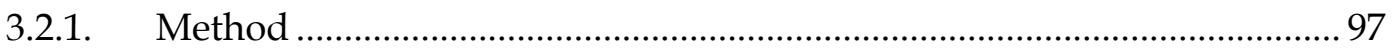

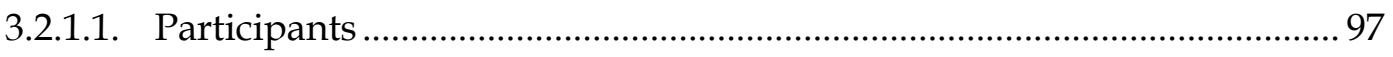

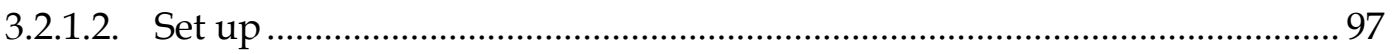

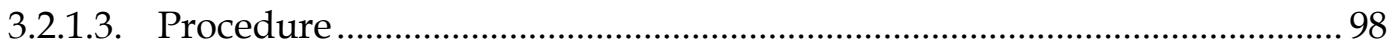

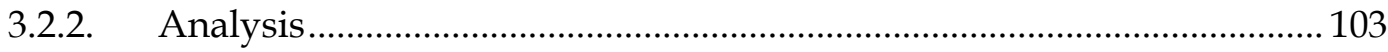

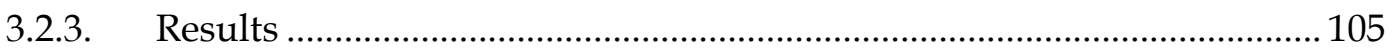

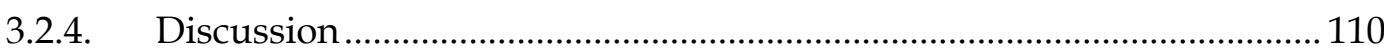

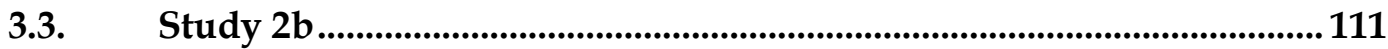

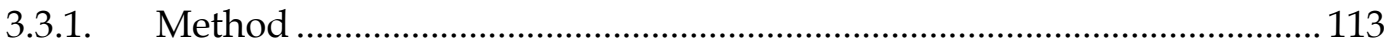

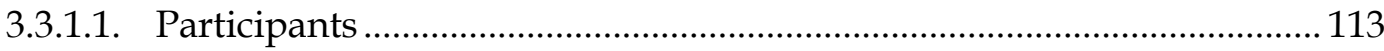

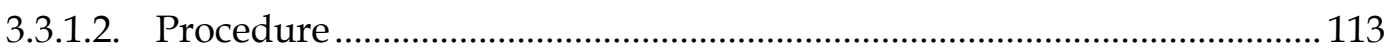

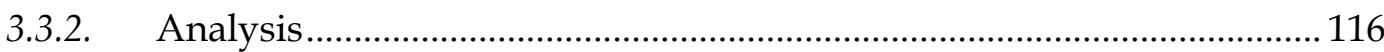

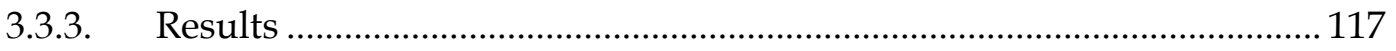

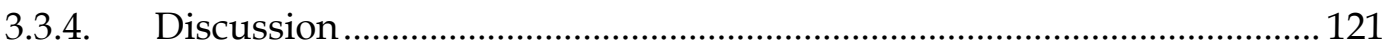

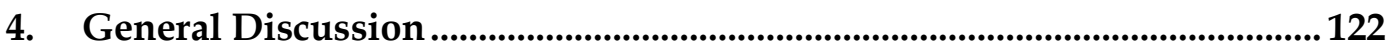

4.1. Selective Encoding of Testimony ..........................................................123

4.2. Default Trust or Rational Inferences? ....................................................128

4.3. On the Role of Contradictory Testimony .................................................... 133

4.4. Early Development of Selective Trust in Testimony.............................. 135

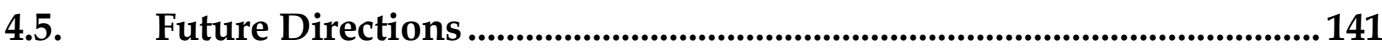

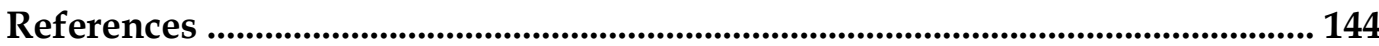




\section{General Introduction}

“Trough others, we become ourselves."

(Vygotsky, 1997)

While growing up, human children have to learn a lot. Much of what they learn is acquired not just trough their own experience, but rather socially, taught by others or trough observation (Bandura, 1986; Vygotsky, 1980). For a lot of abstract knowledge that children require to be a functional member of their community this means relying on somebody else's testimony (Bloom, 1993; Harris, 2012; Woodward \& Markman, 1998). Language acquisition represents one prominent example of such abstract knowledge that children exclusively learn from others, usually from their parents, caretakers and siblings (Akhtar \& Tomasello, 2000). Apart from that, children are able to learn through the testimony of others about facts that are not available to first-hand experience (Boyd, Richerson, \& Henrich, 2011; Harris \& Lane, 2014). This includes such basic facts as that we live on a globe, a round planet called "earth". Although the observational evidence would rather suggest a flat earth, children come to understand this fact early despite a lack of direct experience, trough testimony of others (Harris \& Koenig, 2006; Harris \& Lane, 2014; Vosniadou \& Brewer, 1987). However, our fellow human beings are not always the most reliable relays of information. Even despite their best intentions, people are sometimes convinced of a falsity. At other times, they want to play a joke or sometimes even consciously lie to us. How children react to 
testimony from such sources they deem unreliable is ambiguous - both in everyday experiences with their parents and caretakers, and also in empirical research: Sometimes children appear highly gullible, for example when confronted with ironical utterances or fairy tales (for a review see Pexman \& Glenwright, 2007). This may be unsurprising assuming that children are usually faced with reliable testimony from their parents. After all, parents generally should have an interest to provide their children with true information. Therefore, initially relying on any testimony, regardless of its content, seems like a reasonable heuristic for children to use. Other times however, children appear very proficient in selectively disregarding testimony from others, for example if they want to avoid to clean their room, or when someone is clearly trying to deceive them (DiYanni, Nini, Rheel, \& Livelli, 2012). Developmental research identified children between the ages of three to four to become competent at making use of several indicators when they decide whether or not to rely on another person's testimony (for reviews see Harris, 2012; Mills, 2013). To mention just some examples, when relying on testimony from adults, 4- to 5-year-olds have been found to consider the familiarity of the other person (Corriveau \& Harris, 2009a), and 3-year-olds pay regard to the certainty of that person (Birch, Akmal, \& Frampton, 2010; Sabbagh \& Baldwin, 2001). But what if indicators like these are missing in a given instant? After all, many everyday interactions lack apparent information about another individual's reliability. In situations like these, we may consider our previous encounters with the given person. Has he or she ever lied to me, or unwillingly given me wrong information? Indeed, developmental research shows that children at four years of age already 
possess this ability, although the underlying mechanisms are still subject of debate (Birch, Vauthier, \& Bloom, 2008; Koenig, Clement, \& Harris, 2004; Koenig \& Harris, 2005; Pasquini, Corriveau, Koenig, \& Harris, 2007; Sabbagh \& Shafman, 2009). In this thesis, I investigate the mechanisms behind preschooler's selective trust in testimony of others - with a particular focus on the potentially moderating role of context. Further, I investigate how the ability to selectively trust others develops in human infants below the age of three. While preschoolers have been shown to be able to assess the reliability of a particular person (e.g. Birch et al., 2008; Koenig et al., 2004; Koenig \& Harris, 2005; Koenig \& Harris, 2007), studies on young infants are inconclusive to date (Koenig \& Woodward, 2010; Krogh-Jespersen \& Echols, 2012). In the following, I first explore the origins of human social learning and highlight how social learning is prevalent in several species, yet human highfidelity social learning is unique in the animal kingdom. Humans pay a lot of attention to the social dimension of social learning, which leads them to consider not just the outcome of a model's behavior but also how and by whom it is demonstrated. Then I briefly examine precursors of selective social learning in nonhuman animals, especially with regard to reliability inferred from prior interactions. To explore the developmental trajectory of selective trust in testimony, I examine commonalities and differences in infants' and children's appraisal of sources of testimony. As the literature to date is equivocal, I investigated in my own empirical research whether infants - like preschoolers - assess the reliability of particular individuals based on the accuracy of prior utterances and apply this inference to novel testimony from the same individual. Subsequently, I explore the 
underlying mechanisms of children's selective trust in testimony. Some researchers assume differences in encoding of testimony based on the accuracy of the source to be responsible for children's selectivity. In my empirical research, I thus investigated whether children generally encode information differently depending on its source. However, children do not always discount information from unreliable sources. In this thesis, I thus contrast how children sometimes react gullibly or selectively to testimony from unreliable sources based on the circumstances. In my own studies, I explored whether this gullibility is the result of a bias to default to trust individuals despite children being aware of their unreliability, if there is no competing information available. This would suggest that children's selectivity might be triggered by contextual considerations that lead children to assume a contradiction between testimony from previously reliable and unreliable sources. On the other hand, it could be due to rational inferences leading children to initially rely on questionable information if there is no better alternative. This would suggest that children retain accuracy information even if they rely on testimony from unreliable sources and that they can use this information to adjust their reliance at expression rather than just at encoding. I hope that this thesis will help us get a better understanding of the early development and mechanisms behind children's trust in testimony. 


\subsection{Origins of Social Learning}

In our human society, we frequently rely on information from others. We even send our children to institutions where professionally trained adults educate them about various subjects we deem important in order to become functional members of our community. The process by which we learn from other individuals rather than through own experience is called social learning. In humans, social learning is assumed to be foundational to the accumulation of cultural knowledge by transmission across generations (Dean, Vale, Laland, Flynn, \& Kendal, 2014; Richerson \& Boyd, 2005; Tennie, Call, \& Tomasello, 2009). While the scope of human social learning as foundation of our society and culture is certainly unique in the animal kingdom (Whiten, McGuigan, Marshall-Pescini, \& Hopper, 2009), many other animal species rely on information derived from others. For example chimpanzees, rats, monkeys, birds, even fish and octopuses learn not just from own experience, but also from observing others, suggesting social learning may have evolved as adaption to various ecological niches (Gariépy et al., 2014). However, the way animals learn from others is very different from that of humans. Social learning entails that decision makers use sensory inputs generated by others as sources of information (Gariépy et al., 2014). In most non-human animals however, this is likely governed by the same process that enables them to learn through associations or statistical inferences with no consideration that particular conspecifics generated these sensory inputs (Heyes, 1994; Heyes \& Pearce, 2015; Heyes, 2017). In the following paragraphs, I want to lay out some of the situations 
were animals rely on information derived from conspecifics and how it differs from the way humans learn from others.

\subsubsection{Social learning in non-human animals}

As a way to bypass the need for the many iterations of individual experiences required for individual learning, several species have evolved a capacity to rely on information provided by others to guide their learning (Laland, 2004). However, these forms of learning deviate from human social learning. We engage in "true imitation", which entails that the individual which copies another's behavior actually attended to and learned from the demonstrator (Horner \& Whiten, 2005; Whiten et al., 2009). Even if non-human animals may appear to learn from others, it is not said that this involves considerations about the particular demonstrator. Social learning in non-human animals rather constitutes "learning in a social context", where the information they learn is provided or highlighted by a conspecific instead of acquired through own experience, than actual "learning from others" as in humans.

For example, an ape might display the same behavior as another ape - like using a rock for nut-cracking - simply because of the increased saliency of the rock that had just been used by its fellow. That is, a demonstrator behaving in a certain way towards a certain object might enhance the probability of a similar behavior in another present animal - simply by highlighting the used objects. In animals, 
many cases of social learning can possibly be explained by the process of "stimulus enhancement", e.g. rhesus monkeys learning to fear snakes (Heyes, 1994; Nagell, Olguin, \& Tomasello, 1993).

Another low-level explanation for social learning in animals can be found in external constraints on individual innovation. For example, different cohorts of chimpanzees have developed different tools in order to extract ants from their hives (Galef \& Laland, 2005). In Gombe in East Africa, chimpanzees are using long wands and swipe the biting ants off the stick with their hand. In Tai in West Africa, they use short sticks and pull the stick through their mouth to feed of the ants. Theses geographic variations in chimpanzee's tool use for ant dipping have been interpreted as a cultural phenomenon based on social learning (Whiten et al., 2001). Later research however revealed that the different types of sticks and ingesting behavior are confounded with the ant species' distinct biting behavior (Humle \& Matsuzawa, 2002). It is thus likely, that apes learned to use sticks that are most appropriate in their respective environment, without necessarily considering the behavior of conspecifics.

However, animals also have been observed to engage in copying behaviors that cannot solely be explained by processes of stimulus enhancement or reiterated innovation based on environmental constraints. When witnessing another ape cracking a nut with a rock, observing apes might not just be drawn to the rock because of stimulus enhancement, but actually learn something about the environment as a result of the other's behavior. This form of social learning, which 
has been termed "emulation" (Tomasello, 1990, 1996; Whiten et al., 2009), differs from stimulus enhancement in that the attention is not merely drawn to a stimulus that is manipulated but also to the outcome of this process - for example that food has been extracted from the inside of the nut.

As opposed to true imitation however, where the observer copies and thus learns about the actual process that is demonstrated, emulation learning only entails that the observer understands the change of state in the world caused by the manipulations of the other individual and tries to achieve the same outcome, without detailed consideration of the demonstrated process (Tomasello, 1996). When the demonstration of a behavior includes several causally irrelevant actions, chimpanzees tend to skip those irrelevant actions (Horner \& Whiten, 2005). Observing chimpanzees tended to reproduce irrelevant actions when their causal relevance was unclear, but to ignore them when causal ineffectiveness was obvious. This suggests that chimpanzees are mainly focused on the desired outcome and only secondarily on the demonstrator's actions. Similarly, Call, Carpenter, \& Tomasello (2005) found that chimpanzees preferentially focused on the outcomes of problem-solving activities and tried to reproduce those, while human children preferentially focused on and imitated the actions of the demonstrator. This shows that while non-human animals might learn from conspecifics in ways that exceed stimulus enhancement or repeated constrained innovation, human infants pay a lot more attention to the demonstrator while non-human animals mainly focus on the outcome. 


\subsubsection{Differences to human social learning}

Non-human animals learn from observing others, but for most species, others are just a means to an end that they exploit in order to improve their decision-making. Processes underlying social learning in non-human animals are likely similar to the ones that guide their non-social learning, just that the information came from a conspecific, either in form of making certain types of tools salient or highlighting an outcome that observers can try to copy. Human children and already infants pay a lot of attention to the social dimension of social learning. In fact, this probably constitutes the biggest difference between how human learn from others as opposed to how non-human animals learn from conspecifics. In contrast to the low-fidelity copying found in other animals, humans have a tendency for highfidelity copying and consider not just what is being demonstrated but also who demonstrates it and how a demonstrated result is achieved.

In contrast to the chimpanzees in the study by Horner and Whiten (2005), human children tend to reproduce even obviously causally irrelevant steps with highfidelity when learning from others. In a study by (Lyons, Young, \& Keil, 2007), children were exposed to an action demonstration in which the experimenter extracted rewards from several transparent boxes, while using both relevant and irrelevant actions. While the children could identify both relevant and irrelevant actions without difficulty, they continued to reproduce the whole procedure including the irrelevant actions when tested later. Even explicit instruction to discount actions that were not instrumental in opening the box did not eliminate 
imitation of those actions. This high-fidelity copying of overtly irrelevant actions has been termed "overimitation" and could be replicated with participants from diverse cultural backgrounds (Nielsen, Mushin, Tomaselli, \& Whiten, 2014; Nielsen \& Tomaselli, 2010).

By focusing not just on the outcome of a behavior but rather on the behavior itself and trying to imitate every step of the process, human children are equipped to readily absorb important aspects of their culture (Dean et al., 2014; Tennie et al., 2009). They appear to understand the model as intentionally communicating more than just how to obtain a certain goal. Children thus seem to understand the demonstration as entailing a normative action, they ought to conform to - even if they are uncertain about the instrumental usefulness of each individual step (Kenward, Karlsson, \& Persson, 2011; Kenward, 2012; Keupp, Behne, \& Rakoczy, 2013; Keupp, Behne, Zachow, Kasbohm, \& Rakoczy, 2015).

A further motivation could be of affiliative nature: children might be motivated to copy the causally irrelevant actions in order to affiliate with the model and encourage the model to like them (Meltzoff, 2007; Nielsen \& Blank, 2011; Nielsen, Simcock, \& Jenkins, 2008; Over \& Carpenter, 2012). Instead of seeking the causally most effective solution, children thus seem to be inclined to act according to "what others do", the conventions of the culture they live in. In this manner, process copying, i.e. high-fidelity copying with close attention to the demonstrated process, can lead to the accumulation of useful modifications ("ratchet effect") by being passed on to the next generation, even if their functional utility is opaque (Tennie 
et al., 2009). Product copying (i.e. emulation), in contrast, can only lead to local changes in behavioral traditions (Tennie et al., 2009). Thus, children's high-fidelity copying is likely to be integral to the cultural transmission of traditions in humans. It is probably unique to humans (Tomasello, 2003).

To conclude, the main differences between human children and non-human animals is human's attention to the social dimensiom of social learning. Human children attend to and focus on the process of teaching rather than simply on the instrumental value of what is being taught. While non-human animals have been shown to use emulation learning to reach the same goal as their model, only humans appear to pay close attention to how a model does something and draw inferences about why she demonstrates it in a particular way. In the eyes of children, information provided by others constitutes not just the means to a certain end, but rather appropriate behavior within their social community. This focus on the process of how information is provided and the high-fidelity in the reproduction of socially conveyed information is at the heart of the cultural transmission of traditions in humans. While animals have been shown to learn in a social context, humans specifically learn from other individuals.

\subsection{Origins of Selective Social Learning}

Evolutionary models suggest that indiscriminately relying on social learning is not adaptive, as it would lead to a decreasing proportion of asocial learners producing 
reliable information (Laland, 2004). Rather, learners need to be selective with respect to the circumstances under which they rely on social learning and the individuals from whom they learn. In general, learning is selective if an individual does not learn from every event they are exposed to (selectivity of encoding) or if what they learned is not always expressed in their behavior (selectivity of expression) (Heyes, 2017). This applies to both social and asocial learning. Even in asocial learning, non-human animals exhibit such selectivity.

Selectivity in encoding can for example be observed in animals showing the "blocking effect" (Kamin, 1969). When presented with a compound stimulus, a tone and a light, predicting a food reward, rats do not encode the predictive relationship between the tone and the reward, if they have already been trained to expect a food reward in response to the light stimulus only. Rats thus show selective encoding in that they did not learn about the relation between tone and reward.

Experiments on "latent learning" further show that what animals have learned is not always expressed in their behavior (Tolman \& Honzik, 1930). When rats are provided with several opportunities to explore a maze without a food reward, they show no evidence of learning the lay-out of the maze. However, when subsequently a food reward is introduced at the end of the maze, rats that had the opportunity to explore the maze beforehand perform significantly better than rats that did not explore the maze before. This suggest selectivity of expression in that 
learning did not immediately manifest in a change of behavior before the introduction of a food reward as motivation.

Animals also use socially transmitted information selectively, that is they are selective in when to copy others' behavior as well as whom to copy. It is however unclear whether this selectivity relies on mechanisms that emerged specifically to enable the selective consideration of socially conveyed information. Alternatively, their selective social learning may be based on domain-general mechanisms that are as well responsible for selectivity in asocial learning (Heyes \& Pearce, 2015; Heyes, 2016, 2017).

\subsubsection{Selective social learning in animals: When to copy}

Examples for selective social learning can be found in species quite distant from "homo sapiens". Certain species of fish, for example, generally disregard public information about the profitability of food patches in favor of their own foraging experience. If that experience however lacks reliability or currentness, they draw on information from others (van Bergen, Coolen, \& Laland, 2004). This shows that they are able to switch conditionally between reliance on own information or information from others, depending on the respective reliability of such information (Galef \& Laland, 2005).

Another factor that determines whether animals rely on information from others is the cost of own experience. For example, learning to avoid predators by own 
experience would likely be very costly for an individual. On the other hand, information by others might be less accurate than own experience. Theoretical models suggest that this trade-off makes learning from others when individual learning is costly an adaptive strategy (Boyd \& Richerson, 1988, 2005). Empirical findings, for example in European starlings further support the prevalence of this strategy (Templeton \& Giraldeau, 1996). Starlings observe the foraging success of others and use this information to decide whether or not to stay in a foraging spot. However, they only use information from others when the quality of the spot is difficult to acquire by own efforts.

\subsubsection{Selective social learning in animals: Whom to copy}

Theoretical models of the evolution of social learning further hold that to be an adaptive strategy, animals should not only be selective in when to copy, but also in whom to copy (Laland, 2004). According to the concept of directed social learning, characteristics of demonstrator and observer (such as the social rank, sex, or age) critically affect the probability of social learning (Coussi-Korbel \& Fragaszy, 1995). In support of these theoretical assumptions, non-human animals have been found to employ several strategies leading to selective reliance on others. Several species, such as guppies (Laland \& Williams, 1997) or Norwegian rats (Chou \& Richerson, 1992) for example, are more likely to adopt the behavior displayed by others the higher the number of individuals already displaying said behavior. 
Besides selectively copying behaviors that are frequently displayed, theoretical models propose selectively copying successful individuals to be adaptive (Boyd \& Richerson, 2005). Empirical studies on different species support this hypothesis. In redwing blackbirds for example, the likelihood that individuals will adopt a foodpreference via social learning is influenced by whether the observed individual becomes ill or remains healthy (Mason \& Reidinger, 1982). In bats, those individual who are unsuccessful in locating a food source by themselves follow more successful individuals (Wilkinson, 1992). And chimpanzees preferably follow an informed conspecific with knowledge of the location of concealed food as compared to an uninformed conspecific (Menzel, 1973, 1974). Other strategies that non-human animals have been found to rely on for model selection are to copy closely related individuals, to copy "friends" or to copy older individuals (for a review see Laland, 2004). Abstractly speaking, animals adjust their model selection based on properties immediately predictive of success rather than relying on a consideration of the particular individual they are copying. They rely on others especially when the costs of individual experience and the benefit of this reliance are apparent during the crucial time when the model provides information.

\subsubsection{Origins of selective trust in testimony}

Non-human animals do not just rely on observed behavior of others, but also on utterances of others, which can be understood as the origins of trust in testimony. Vervet monkeys for example rely on alarm calls by conspecifics who warn them 
from different predators (Seyfarth, Cheney, \& Marler, 1980). Some animal species have been shown to be selective in reliance on such utterances. Dogs and great apes, for example, adjust their reliance on communicative utterances of an individual depending on the accuracy of prior utterances of the same individual. Domestic dogs quickly cease to follow pointing gestures by an unreliable informant in an object choice task, while they subsequently still follow gestures of a naïve informant in the same task (Takaoka, Maeda, Hori, \& Fujita, 2015).

Further, it has been found that great apes adjust their gaze following behavior depending on the accuracy of an individual's communicative cues even in a subsequent distinct task (Schmid, Karg, Perner, \& Tomasello, 2017). These findings speak for the idea that animals' selective reliance on social information does not necessarily depend on model characteristics that are visible in the very moment of communication, but rather that they can use their experience of prior behavioral reliability to adjust their reliance on other individuals. It is, however, unclear whether these examples of selectivity in animals' social learning rely on mechanisms that evolved specifically to enable the selective consideration of socially conveyed information. Alternatively, animals' selective social learning might be based on domain-general mechanisms, also present in asocial learning (Heyes \& Pearce, 2015; Heyes, 2016, 2017). They likely track the predictive relationship between events and if the situations is predictive of reduced success, they selectively learn less (Heyes \& Pearce, 2015). 
In the next paragraphs, I will explore how human children achieve selectivity in trusting the testimony from others. As a prototypical case of social learning, I will focus on children's learning of novel words. I further will compare 2- and 5-yearold children to explore the developmental trajectory of selective trust in testimony.

\subsection{Early Development of Selective Trust in Testimony}

In my project, I investigated how children and infants selectively learn novel labels for unfamiliar objects, to model the process of language acquisition. Language acquisition is an especially interesting case of social learning, as learning the meaning of novel words constitutes prototypical social learning: The connection between words we use and their meaning is arbitrary and opaque (Bloom, 2000). As a result of that, we cannot become acquainted with the meaning of words during early infancy by any other means than social learning, typically taught by parents, caretakers, and siblings, but also through observation of third party conversations (Bloom, 2000). It is important to be selective when learning the meaning of novel words, as if we acquire errors it will be difficult to be understood by our language community later on.

Children have been shown to incorporate diverse characteristics of the source of testimony in their decisions about whether or not to learn a novel label (for reviews see Harris, 2012; Mills, 2013). Some of these characteristics are apparent at the time when the source provides testimony, such as how old or how familiar to the child 
the individual is (Corriveau \& Harris, 2009a; Jaswal \& Neely, 2006). Children use these features to estimate whether someone's testimony will likely represent the conventional "truth" within the children's language community. However, because these features are apparent, children do not necessarily need to rely on sophisticated inferences. Instead, those apparent features can directly serve as indication that what is being said likely bears little relevance within a child's community. This reliance on apparent model characteristics, which are immediately predictive of the relevance of the behavior, is reminiscent of nonhuman animals' model selection.

In everyday interactions with other individuals however, there are often no such obvious signs of their reliability. Instead, children can rely on information they gathered about the reliability in prior interactions with the same person (e.g. Birch et al., 2008; Koenig et al., 2004; Koenig \& Harris, 2005). Investigating how children use deduced reliability from prior interactions to achieve selectivity in their reliance on testimony thus is a research setting of high ecological validity. And, as I want to lay out in the following paragraphs, it is based on inferences about particular individuals rather than mere assessment of the relevance of what is being taught. 


\subsubsection{Reliability attribution in preschoolers}

At 3 to 4 years of age, children are able to make person-specific inferences about the reliability of an informant, rather than just surface generalizations of "correct" and "incorrect" behavior (see also Koenig \& Harris, 2007). In a study by Koenig, Clement, and Harris (2004) 3- and 4-year-olds watched two speakers labeling familiar objects either consistently correct or incorrect. Children were asked to judge who of the two speakers said something right and who said something wrong. If children answered these questions correctly, they were more likely to accept the accurate speaker's novel label for an unfamiliar object as compared to the label provided by the inaccurate speaker. This finding is telling in several regards. First, it shows that children can encode, monitor and recall source information, when prompted to do so. Furthermore, the fact that specifically those children who were able to assess speaker's accuracy correctly were selective in their trust suggests that successful monitoring of source accuracy influences selective trust. Similarly, in a study by Hermes, Behne, and Rakoczy (2015) children chose models selectively in accordance with traits corresponding to the task at hand (Hermes, Behne, Bich, Thielert, \& Rakoczy, 2018). Namely, children preferred a strong model for strength-related tasks and a knowledgeable model for knowledge-related tasks. This pattern of selective model choice held true only for those children who at the conclusion of the experiment were able to explicitly identify the attributes of both models, again suggesting that successful source monitoring plays an important role in selective trust. The representation of the accuracy of these sources seems to be at least fairly stable, as 3- and 4-year olds 
continue to display selective trust several days after the initial encounter with the inaccurate and the accurate informant (Corriveau \& Harris, 2009b).

Preschoolers have consistently been shown to generalize these inferences to future novel testimony (for reviews see e.g. Harris, 2012; Mills, 2013). Birch, Vauthier, and Bloom (2008) showed that even without prompting explicit judgements of speaker accuracy, 3- and 4-year-olds used prior (in)accuracy at labelling familiar objects to assess the reliability of novel testimony. Children preferred a novel label for an unfamiliar object provided by the previously accurate informant to that by the previously inaccurate informant. Furthermore, the authors could show that children use the reliability assessment of novel claims to guide their learning. Children were confronted with a novel object labelled by the accurate informant and another novel object labelled by the inaccurate informant with the same label as used by the accurate informant. When children were then asked to select the referent of a different novel label they had not heard before, children selected the object labelled by the inaccurate informant. The authors conclude that children must have had fully encoded the label used by the reliable informant as referring to the object she had labelled. Children then used this information to infer the meaning of the novel label as referring to the object labelled by the unreliable informant. This shows that at the age of 3 to 4 years, children not only assess the reliability of an informant based on his or her history of accuracy when prompted to (see Koenig et al., 2004), but also do so spontaneously. Further, they use this 
information to assess the reliability of novel testimony and to guide their subsequent learning.

\subsubsection{Selective trust in testimony in infancy}

At least above 4 years of age, human children deduce the reliability of a particular person from the accuracy of past testimony. They use this person-specific inference to assess the reliability of novel testimony in the future and to guide their learning from testimony from that person. But what do we know about selective trust in testimony in infants? Do they employ similar strategies? Or is their selective social learning more similar to that of non-human animals, that is they learn less in situations that are predictive of reduced success and do not involve considerations about the particular individual they are learning from? Findings form studies with children below the age of three are to date inconclusive.

\subsubsection{Error detection}

Already at 16 months of age, human infants are able to detect a mismatch between an image of a familiar object and a label a human speaker uses to refer to that object. Infants show increased looking time towards a human speaker when the speaker produced false rather than true labels (Koenig \& Harris, 2007). However, when the source which uttered the incorrect label was an inanimate audio speaker, infants 
did not look longer at the source during false versus true labeling. This goes to show that infants' attention to accuracy is not just based on a failed association between the object and the heard label, but rather on an interest in the source of the message (Koenig \& Echols, 2003). Furthermore, infants tried to correct the human speaker, uttering the correct label for the object, but not the inanimate source. To exclude the possibility that the violation of an associations between human speakers and accurate labeling was the driving force behind infants increased looking time, infants were then presented with a human speakers either facing the infant or facing backwards. Now, infants did not appear surprised when a speaker produced false labels while gazing away from objects, only when the speaker was facing forward. Taken together, these studies show that infants are sensitive to the epistemic conditions under which people typically name objects correctly and when they commit errors (Koenig \& Harris, 2007). They are not only attending to the accuracy of the testimony, but also to characteristics of the source of that testimony. If the speaker is human, they appear to be surprised by false labels when the speaker had perceptual access to the labelled object and even offer corrections. This goes to show that infants recognize mismatches of testimony with their own experiential record early in development. However, from these studies showing infants being able to detect a mismatch of own knowledge and others' testimony, it is not yet clear whether infants really evaluate the trustworthiness of the informant. In order to be selective in their trust in testimony, children must further be able to discriminate between different speakers, encode person-specific information about accuracy and hold it in memory (Koenig \& Harris, 2007). 


\subsubsection{Source monitoring}

While this shows that infants are already sensitive to the distinction between accurate an inaccurate assertions, it is unclear whether - like preschoolers' - they can use this sensitivity to attribute reliability to particular individuals. Alternatively, their perceived selectivity could be based on reduced learning when expecting reduced success like in non-human animals. Maybe infants only engage in an appreciation of group-based differences, i.e. they only make categorical judgements (see preference for "adult"- informant over "child"-informant, Jaswal \& Neely, 2006). On the other hand, the fact that infants learn less from an inaccurate source could simply be a result of confusion due to unusual cues or due to the violation of the expectation that testimony by human speakers usually corresponds with infants own knowledge.

There are a few studies, which suggest that already below 4 years of age children are able to assess and keep track of the reliability of individual speakers. Even for infants at the age of 24 months there are signs that they treat individual speakers differently. Koenig \& Woodward (2010) found that 24 month olds react differently when confronted with a previously accurate as opposed to a previously inaccurate informant. They were more likely to select the target object in response to a previously accurate speaker as compared to an inaccurate speaker. In a follow up experiment, when tested by an unknown third person after being taught by an inaccurate one, children showed similar high rates of reliance on the naive as on the reliable informant. According to the authors, this suggests that it is unlikely 
that the reduced learning from the inaccurate informant in the first study was based on general confusion. Similarly, Krogh-Jespersen and Echols (2012) could show that while 24-month-olds do accept a novel (i.e. second) label for familiar objects taught by a previously accurate, knowledgeable or naïve speaker, they did not accept a second label from a previously inaccurate or self-proclaimed ignorant speaker.

On the other hand, there are findings, which suggest that infants might rely on different strategies than preschoolers to achieve selectivity in their trust in testimony. As mentioned, when in the study by Krogh-Jespersen and Echols the objects were novel, children were willing to accept a novel label for the object regardless of the prior accuracy of the speaker (Krogh-Jespersen \& Echols, 2012), even from a previously inaccurate or self-proclaimed ignorant source. Similarly, in the study by Koenig \& Woodward (2010), while infants learned from a previously accurate informant at higher rates than from an inaccurate informant, they selected the target object at rates above chance even when an inaccurate informant had labeled it.

As a whole, the findings for infants below the age of three do not allow for a definite conclusion of whether they use the same strategies in their selective trust in novel testimony that preschoolers use. Some studies show that infants can assess the reliability of novel testimony; others show that they need own knowledge to pit testimony against in order to be able to reject testimony from inaccurate sources. In my project, I investigated whether infants like preschoolers attribute reliability 
to individuals based on the accuracy of their testimony. Furthermore, to explore infants' use of accuracy information on novel testimony to guide their learning we conducted a simplified version of the tasks used by Birch and colleagues (2008) (see 1.3.1. Reliability attribution in preschoolers).

\subsubsection{Single informant paradigms}

Studies investigating selective trust in testimony with young infants typically rely on between subjects designs. Presumably to keep the task demands low, infants are confronted with a single accurate or inaccurate informant who offers novel testimony. However, this particular design allows for alternative explanations for infant's reduced learning from an inaccurate speaker. As infants are used to ostensive cues as markers of situations where typically generalizable information is conveyed (Csibra \& Gergely, 2009, 2011), inaccurate testimony presented with such cues might confuse them. This confusion could in turn lead to reduced learning without an appreciation of the particular inaccurate individual. Studies where the inaccurate informant is the only available source of testimony can tell us whether infants learn in situations where they are confronted with an unreliable informant. In these studies, typically the degree to which infants learned from a reliable informant is then compared to the degree to which they learned from an unreliable informant. But it is difficult to discriminate whether infants actually attribute (un-)reliability to the source of that testimony or whether they simply 
learned less because of situational variables that suggest reduced utility of the information that is conveyed.

In order to try to conclusively investigate whether infants assess particular individuals differently and ascribe reliability to them, I provided infants with both a reliable and an unreliable informant at the same time. I used a video-presentation and eye-tracking to keep the task demands low. In order to exclude several alternatives for infants' selectivity in the literature, I conducted two complementing studies in Study Set 2.

\section{Conclusion}

When confronted with two sources of information that differ in certain characteristics that might be indicative of their reliability, such as the age (Jaswal \& Neely, 2006) or prior accuracy of the informant (Koenig \& Harris, 2005), preschoolers are prolific at selecting the better or more appropriate of the two informants. Moreover, as recent research showed, those children who correctly inferred the relevant traits of the informants were able to use that information to infer their reliability and expertise and to recruit them selectively depending on the requirements of the context (Hermes, Behne, \& Rakoczy, 2015). This shows that preschoolers engage in inferences about the sources of testimony and adapt their reliance on novel testimony from the same source accordingly. But how do infants process this information? Do they engage in inferences about particular sources of testimony in the same way as preschoolers? Or do they simply learn less in 
confusing situations? In Study Set 2 (chronological numeration), I investigated the early development of trust in testimony. As other studies usually confront infants with only a single reliable or unreliable informant, presumably to reduce the task demands, they typically cannot exclude lower level explanations for infants reduced learning from a previously inaccurate speaker. I used an eye-tracking paradigm to keep the task demands low, while at the same time being able to confront infants with both a previously accurate and a previously inaccurate informant.

In the next paragraphs, I want take a closer look at the underlying mechanisms of children's selective trust. While they are certainly able to selectively prefer information from a reliable to an unreliable source, it is unclear whether this entails a general difference in encoding of testimony from the two sources. Furthermore, children sometimes rely on testimony from unreliable source, which could be a hint that only specific circumstances trigger children's selectivity. In my own empirical research, I explored children's reliance on testimony by unreliable sources with a focus on differences in encoding and the role of context.

\subsection{Underlying Mechanisms of Selective Trust in Testimony}

At four years of age, children are able to infer from prior interactions who of two speakers is a reliable or unreliable provider of testimony, attribute reliability to particular individuals and use the assessment of novel claims to adjust their 
learning. They do this not only when prompted to judge the reliability of an informant (Koenig et al., 2004), but also spontaneously keep track of informants' history of accuracy and use this information to assess the reliability of further testimony from the same source and to guide their subsequent learning (Birch et al., 2008).

But how do children achieve this selectivity? As mentioned, mainly two points of application of accuracy information in order to be selective are conceivable. Children either use inferences about a source's reliability when provided with testimony from that source and adjust the encoding of such information (selectivity of encoding). Or children encode information from any source, but also retain information about the accuracy and use this inference to adjust their reliance when retrieving and before reproducing testimony from that source (selectivity of expression). There is evidence, which suggests that children encode information differently depending on the reliability of the source. In my own empirical research, I wanted to investigate these differences more precisely in order to discern possible mechanisms of children's selectivity.

\subsubsection{Selective encoding of testimony}

Developmental research shows that when children react to testimony, they consider not just the testimony itself but also reason about the source. When confronted with two sources of testimony that differ in certain characteristics that 
might be indicative of their reliability, children are prolific at selecting the better or more appropriate of the two informants. As recent research shows, those children who correctly inferred the relevant traits of the informants are able to use that information to infer their reliability and expertise and to recruit them selectively depending on the requirements of the context (Hermes et al., 2015). This shows that children engage in inferences about the sources of testimony and adapt their reliance on testimony accordingly. Researchers typically tried to explain the mechanisms underlying children's selective trust in testimony by focusing on how children achieve selectivity in situations when they are selective.

On a low level account, when confronted with testimony from someone who previously made a mistake, children could simply ignore any information that is provided (Kim, Paulus, \& Kalish, 2017; Mangardich \& Sabbagh, 2018). According to this "Blocking" account, children only attend to and process information by a previously reliable informant, further testimony by the unreliable source is consequently disregarded. Children's preference for information by a reliable informant according to this account is thus due to it being the only information they processed and learned.

Alternatively, children may attend to information by both reliable and unreliable informants, but block the processing of semantic information provided by someone who has been unreliable in the past. Such a "Semantic gating" account assumes a top-down gating mechanism that allows children to consider characteristics of the source during the encoding of information in order to guide 
their learning (Sabbagh, Koenig, \& Kuhlmeier, 2017; Sabbagh \& Shafman, 2009). These assumptions are based on the finding that children learned episodic information from both a knowledgeable and an ignorant speaker, but semantic information only from a knowledgeable speaker (Sabbagh \& Shafman, 2009). When asked about the labeling episode ("Which object did I say is the ... ?") children were able to answer correctly both whether the speaker had proclaimed ignorance or knowledge about the information in question. However when asked for reproduction of that information ("Which object IS the ... ?") children only relied on information from the knowledgeable, but not the ignorant speaker. This shows that children retained episodic information about a labeling event even for the unreliable informant, but have not learned a novel label for a novel object from her. Both of these accounts, Blocking and Semantic Gating have in common that they assume children to be selective when acquiring novel information, i.e. via a (top-down) process during the encoding of information.

In my project, I investigated whether children generally encode testimony from previously accurate and inaccurate informants differently. By using tasks with varying difficulty, I explored further how exactly those differences manifest. Assuming that children block any further information from an unreliable source, we would expect to find no evidence for any encoding of such information. Attention and encoding of episodic information about the labelling event, as assumed by the Semantic gating account, however should at least let children draw the inference that the object in question had been labelled before. However, 
both accounts assume that infants generally learn less from an unreliable source. In Study Set 1, I focused on these assumptions in order to differentiate between Blocking and Semantic Gating and to investigate whether children generally encode information from reliable and unreliable sources differently.

\subsubsection{Default trust in testimony}

Recent research suggest that children might not always discount information from an unreliable source. Even at an age where children certainly are capable to selectively disregard testimony by previously inaccurate informants, they sometimes appear highly gullible. In fact, children's reliance on testimony by unreliable informants is sometimes so strong, that some researchers assumed it to be a default reaction to testimony. For example, children sometimes ignore obvious errors of adult's testimony about the contents of a box (Mascaro \& Sperber, 2009) or the location of a reward (Jaswal, 2010a; Jaswal, Croft, Setia, \& Cole, 2010; Vanderbilt, Liu, \& Heyman, 2011) and continue to trust subsequent testimony from the same informant. They even continue to follow an adult's testimony about the location of a hidden toy, when the adult had repeatedly indicated the wrong of two locations (Jaswal et al., 2010), and when the adult's testimony conflicted with other evidence about the toy's location (Jaswal, 2010a). Given that children were not mislead in the same way by a sticker indicating the incorrect location suggests a failure to specifically discount false testimony. These studies seem to contradict the notion that children are selective in their trust in testimony, leading 
Jaswal and colleagues (2010) to suggest that preschoolers employ a specific bias to trust testimony even from unreliable speakers which they called 'default trust'.

\subsubsection{On the role of contradictory testimony}

So under which circumstances do children rely on testimony by unreliable sources? For one, adding to the notion of children as prolific interpreters of testimony, children continue to trust unreliable informants when their inaccuracy can be excused by external factors, such as lack of perceptional access (Nurmsoo \& Robinson, 2009).

Other studies however suggest that children might not always consider prior inaccuracy of the source when relying on testimony. A prerequisite for children's reliance on unreliable informants appears to be whether the information provided by them is novel, i.e. not contradicting anything the children themselves already know. When an unreliable speaker offered a novel label for a novel object, children relied on her testimony at rates above chance (Krogh-Jespersen \& Echols, 2012). On the other hand, when presented with a second label for a familiar object, children did not endorse this label when it was taught by a previously unreliable speaker. While the participants in this study were only 24 months old, other studies with children above three years similarly found their participants relying on novel information from an unreliable source (Kim et al., 2017; Vanderbilt, 
Heyman, \& Liu, 2014), but not if it was in contradiction with children's own prior beliefs (Li \& Yow, 2018).

In general, it seems to be an important factor influencing whether children rely on testimony by an unreliable informant if there is a better alternative to that testimony available during the encoding of such testimony. Additionally to own prior knowledge, as in the case of second label learning (Krogh-Jespersen \& Echols, 2012), this better alternative can constitute testimony from a third person. In a typical study showing children's selective endorsement of testimony by a reliable source, an informant who was introduced as reliable and another informant who was introduced as unreliable offer two different labels for the same object (Koenig et al., 2004; Koenig \& Harris, 2005), or the same label, but for two different objects (Birch et al., 2008; Scofield \& Behrend, 2008). Here, as children expect each object to have only one label ("mutual exclusivity"; (Markman \& Wachtel, 1988)) testimony from the two sources is in contradiction. This contradiction is further reinforced by the context of the situation. When they proceed to teach the children novel labels for novel objects, children have typically just seen both informants either use the same familiar label, but to refer two different familiar objects, or both use different familiar labels to refer to the same familiar object (Birch et al., 2008; Koenig et al., 2004). Thus, contextual considerations might lead children to expect similarly that the novel information provided by the same informants conflicts in the same manner as the familiar information. Children appear to resolve this perceived contradiction by preferring information from the more reliable source. 
On the other hand, if there is no contradictory testimony from a more reliable source, children appear willing to rely on and thus encode testimony by an unreliable informant. Studies that use a single informant design, where children are either confronted with a consistently accurate or inaccurate informant show that when the testimony from an unreliable speaker was the only available information, children relied on testimony from the unreliable informant (Jaswal, 2010a; Kim et al., 2017; Koenig \& Woodward, 2010). Even when a previously inaccurate informant was paired with an accurate informant, children continued to trust the unreliable informant, as long as they perceived testimony from the unreliable informant not to conflict with the testimony from the more reliable source. They disregarded the label offered by an unreliable informant when a more reliable informant provided an alternative label, but endorsed it when the unreliable informant was the only source of information (Kim et al., 2017; Vanderbilt et al., 2014). In fact, if the testimony by the previously accurate and the inaccurate informant was not in contradiction, i.e. if they both offered two different labels for two different objects, children appear to learn from both informants to an identical degree (Kim et al., 2017). Kim and colleagues (2017) found that children apply a novel label to other objects with decreasing similarity to the original referent to the same degree whether the original referent had been labelled by a reliable or unreliable source. 


\subsubsection{Contradictory testimony as trigger of children's selectivity?}

This is interesting as it could suggests that a perceived contradiction in testimony from sources with different reliability might trigger children's selective encoding of such information. If there is no such contradiction, children appear to learn from both informants equally. But does that mean that children's bias to trust leads them to rely on information from the unreliable source and discount accuracy information completely when testimony by an unreliable source was not contradicted? Or is children's reliance on uncontested information from unreliable sources based on rational inferences? This would mean that children rely on information from an unreliable source despite knowing about their inaccuracy, because it is the only information they have, and questionable information is still better than no information. In other words, can children retain accuracy information even in case of uncontested testimony from unreliable sources and still be selective at expression?

The paradigms, studies have used to investigate learning from uncontested testimony by an unreliable source usually investigate the degree to which children learned from the unreliable source. This is then typically compared to the degree to which children learn from a reliable source. For example in the study by Kim and colleagues (2017), children were asked to decide whether objects with decreasing similarity from the original referent would still be referred to with the original label. If the unreliable source had called an object a "tog" children were repeatedly asked to decide for different objects "Is this a tog?". These paradigms 
however offer the children no inducement to discount testimony by the unreliable source. Rather, already by asking for its reproduction, the testimony by the unreliable informant might gain relevance and thus be reproduced despite the child knowing it came from an unreliable source. Hence, these paradigms cannot conclusively determine whether children under such circumstances are biased to trust and discounted accuracy information. Alternatively, their reliance on testimony from an unreliable source might be caused by rational inferences leading children to rely on questionable testimony rather than not to rely on it, given there was no better alternative available. In this case, children would likely retain accuracy information and could still be selective expression, but decided not to as there was no better option than testimony from the unreliable source. In my project, I investigated how children react to a forced-choice paradigm when a previously reliable and an unreliable informant offered testimony that is not in contradiction to each other. This will help to explore whether children retain accuracy information and can use it to adjust their reliance on testimony between retrieval and reproduction.

\section{Conclusion}

Children learn much of what they learn not through direct experience, but through the testimony of others, especially from parents and teachers. The testimony of others however can be mistaken due to various reasons from mistaken beliefs to deceptive intentions. We know that when children hear testimony they engage in 
rational inferences about the sources and adapt their reliance on such testimony accordingly. In my project, I aimed to identify how children incorporate these inferences in their selective social learning from testimony. Learning is selective if an individual does not learn from every event they are exposed or if what they learned is not always expressed in their behavior. Children can thus be either selective at the encoding of testimony or at the expression. In my own empirical research, I first investigated whether there are generally differences in how children encode testimony depending on the reliability of the source. I compared predictions based on a Blocking account, assuming that children inhibit any information from unreliable sources, with predictions based on a Semantic gating account, assuming that children encode episodic but no semantic information from unreliable sources. As recent research shows that children might not always be selective, I investigated whether their reliance on testimony from unreliable sources is dependent on the availability or lack of contradictory information from a better source during the acquisition of such information. When testimony from an unreliable source is not contradicted by own knowledge or an alternative source of testimony, children appear to learn from the unreliable source to a similar degree as from a reliable source. In Study Set 1, I explored whether that means that children in such circumstances trust in testimony by default and discount accuracy information, or whether they still can adjust their reliance on testimony from different sources when deciding whether to reproduce such information, at expression. This would suggest that children engage in rational inferences and simply rely on uncontested testimony from unreliable source because even 
questionable information is better than no information. Thus, to expand our understanding of children's trust in testimony, the main focus of my project was to test the influence of a perceived contradiction between the two sources compared to uncontested testimony from either source. In the following chapters, I present the empirical studies I conducted for this project. I want to start chronologically by first laying out the studies I did with 5-year-olds to explore the underlying mechanisms of trust in testimony (Study Set 1). These studies further establish my eye-tracking paradigm, which I later used to explore infants' learning from two distinct sources of testimony (Study Set 2). 


\section{Study Set 1: Underlying Mechanisms of Selective Trust in Testimony}

\subsection{Introduction}

We know that when preschoolers react to testimony, they consider not just the testimony itself but also reason about the source. When confronted with two sources of information that differ prior accuracy, children are prolific at selecting endorsing testimony from the better or more appropriate of the two sources (e.g. Koenig \& Harris, 2005). As recent research showed, those children who correctly inferred relevant traits of informants were able to use that inference to estimate their reliability and expertise and to recruit them selectively depending on the requirements of the context (Hermes et al., 2015). These findings suggests that children above the age of four engage in inferences about the sources of testimony and adapt their reliance on testimony accordingly. But how do children use those inferences? The mechanisms underlying their selectivity are not yet fully understood. Do they always encode testimony according to inferences about the reliability of the source? Do they only use those inferences to encode testimony by an unreliable sources differently when a more reliable source offers additional testimony that suggests the unreliable source's testimony might not be applicable? Or can they also use those inference to adjust their reliance at expression?

Some researchers argue that children's selective trust in testimony relies on differences in encoding of testimony depending on the source. If confronted with 
testimony from previously inaccurate sources, children are assumed to either inhibit the acquisition of further testimony from that source or to attend to information but only retain episodic information about the labelling event. However, the context in which this testimony is provided, namely whether the reliable informant offers testimony that can be understood as a better alternative to testimony from the unreliable informant, appear to influence children's selectivity. This could mean that a perceived contradiction in testimony might trigger children's selective reliance on such testimony. In Study Set 1, I aimed to investigate the underlying mechanisms and the role of context on children's selective trust in testimony.

In Study 1a, I presented children with contradictory information by a reliable and an unreliable informant and tested children's information uptake and inferential reasoning using both eye-tracking and interactive tasks. The aim here was to replicate findings of studies with conflicting information (Birch et al., 2008), validate our eye-tracking method and investigate how willingly children are to discount information from unreliable sources.

In Study 1b, I investigated how children generally encode information from accurate and inaccurate informants. The aim of this study was to assess whether and how children generally attend to and remember novel labels provided by an unreliable source and to compare it to the degree to which children learn from a reliable source. This enables us to examine which of the different theoretical accounts for underlying mechanism of children's selective trust can be applied to 
trust in testimony more generally. Do children always block all information from unreliable sources of testimony, as the Blocking account would suggest, even if the information provided is uncontested? Do children attend to such information, but not encode it semantically, as proposed by the Semantic Gating account? Or do they generally attend to and learn from unreliable sources, as long as they are not contradicted by more reliable sources?

In Study 1c, I explored directly whether children's selective disregard of information from unreliable sources is necessarily dependent on the availability of a more reliable source that offers contradictory information during the acquisition of information. I compared in a between subjects design how children treat testimony from reliable and unreliable speakers when it was contradicting to when it was compatible. It could be that children are biased to endorse uncontested information even from unreliable sources and afterwards treat it identically to information from any other source. Alternatively it could be that children may initially use the uncontested information from the unreliable source, given that is the only information available to them at the time, but that the information is somehow tagged as possibly inaccurate. Children could then use this (in-)accuracy information and adjust their reliance at expression.

\subsection{Study 1a}

The aim of Study 1a was to establish the method used in subsequent studies both with preschoolers and infants by replicating findings of studies where a previously 
accurate and an inaccurate informant provide contradictory testimony. I used both interactive forced-choice and eye-tracking paradigms to validate eye-tracking as method to investigate selective trust in testimony. This will enable us to use eyetracking in Study $1 \mathrm{~b}$ as measure to investigate differences in children's encoding of testimony by the two sources. Further, it will validate the use of eye-tracking to explore the early development of selective trust in testimony and infants' appreciation of differently reliable individuals in Study Set 2.

In Study 1a, children watched a video presentation of two female informants labelling familiar objects accurately or inaccurately. Then both informants used the same novel label, but to refer to two different objects. To find out whether and how children rely on this information, they participated in four tasks, two eye-tracking tasks followed by interactive versions of these tasks.

In the eye-tracking and interactive Endorsement tasks, children were presented with the two novel objects that the two informants had referred to respectively, and children were asked to pick out the one, which they thought the label used by both referred to. This replicates typical paradigms where two informants with differing history of accuracy offer conflicting information and children are presented with a forced-choice task (e.g. Birch et al., 2008). Based on findings by these studies, I expected children in the eye-tracking task to look towards the object that had been labelled by the reliable informant upon hearing the label used by both, if they preferentially endorsed information by that person. Similarly, in the 
forced-choice task, children should select the object labelled by the reliable informant as the referent of the label used by both.

To find out to what degree children discount information from an unreliable speaker in the case of a contradiction with information from a more reliable source, I used an eye-tracking version and an interactive version of the Contrast task (Birch et al., 2008). Here, children saw the two objects labelled by the two female informants on screen and then a third male person, asked for the referent of a different completely novel label. Only if children are willing to discount information by an unreliable source in favor of information by a source with unrevealed accuracy, they should expect the novel label to refer to the object labelled by the unreliable speaker, rather than the object labelled by the reliable one. Using an eye-tracking Contrast task, I was interested whether this was already detectable in children's spontaneous looking behavior.

\subsubsection{Method}

\subsubsection{Participants}

Thirty-six monolingual German children $(\mathrm{M}$ age $=65.5$ months, range $=60.8-71.5$ months, 18 female) were included in the final sample of this study. Participants were recruited from a database of children whose parents gave consent to be invited for child development studies. None of the participants had known visual or auditory impairments. Parents gave informed consent for participation of their 
child. Two additional children participated but could not be included in the final sample because of technical difficulties that led to the eye-tracking device not recording gaze data.

\subsubsection{Set up}

For the eye-tracking tasks, I used a SMI Red250 eye-tracker attached to a 24-inch TFT flat-screen monitor with a display resolution of $1920 \times 1080$ pixels. The eyetracker records gaze data at $60 \mathrm{~Hz}$ with an average accuracy of $0.4^{\circ}$ visual angle. During the experiment, the children sat on an adjustable chair that I used to ensure that children's eyes were inside the detectable range of the eye-tracker. Prior to testing, I calibrated the gaze of every child using a 5-point calibration procedure, in which an attention-getter appeared in every corner and the middle of the screen, followed by a 4-point validation procedure. The stimuli - a series of video clips, still pictures and audio recordings - were presented using E-Prime 2.0 as presentation software. The novel objects that children handled, and saw still pictures of, were plush toys representing microbes (a green plush toy, representing Euglena, and a blue plush toy, representing Giardia).

\subsubsection{Procedure}

The session started with a warm-up phase, in which the child played with the two experimenters (E1 and. E2). During this phase, the child was allowed to play for 
up to 20s with either plush toy that would later be named by the reliable and unreliable informants (each toy was presented for 20s). Then for the eye-tracking part of the study, the child was seated in front of the screen, to which the eyetracker was attached, and the seat's height and distance to the screen were adjusted to ensure that children's eyes were inside the detectable range of the eye-tracker to record children's gaze.

Subsequently, each test session consisted of the following phases. First, infants were introduced to both informants and their labelling accuracy in the History phase. In the Acquisition phase, both informant proceeded to provide novel testimony, that is a novel label for a novel object. In our first study, the information provided by the two informants was contradictory, i.e. they offered the same novel label but for different novel objects. Children's appreciation of testimony from the two sources was then investigated in two eye-tracking tasks: the Endorsement and Contrast task. Finally, infants participated in interactive versions of these tasks. As an attention grabber, in the very beginning of the experiment and as a transition scene after the History phase and after the Acquisition phase, a 6 s video of balloons to piano music was included in the study.

\section{History phase}

At the beginning of the video presentation, the children saw a 10 s greeting part to introduce the two informants: a blonde-haired and a brunette female actress appeared in turn, one in the top left corner and the other in the top right corner of 
the screen, with each waving in the camera and saying "hello". Then children saw eight film clips (four with each informant) in which the informant labelled a familiar object, to familiarize children with the (un-)reliability of the informants. Children first saw one of the informants label three objects in turn, then the other informant labelled those same three objects in reversed order, followed by each labelling a fourth object. As familiar objects, I selected items whose labels children are highly familiar with (apple, house, dog, ball). If the speaker was reliable, she used the correct label to refer to the objects visible on screen ("Oh, guck mal! Das ist ein Apfel, ein Apfel!" - “Oh, look! That's an apple, an apple!”), if she was unreliable the label she used did not refer to the object present on screen, but was from the pool of familiar labels used by the reliable informant during the experiment (see Figure 1 I.). Additionally, I made sure that label she used did not correspond to the object presented to infant directly before or after the current object. The identity of the reliable informant and order of the informants was counterbalanced across children.

Each of the film clips of the History phase lasted 10s and showed the following sequence. First, a familiar object appeared in the bottom middle of the screen, then one of the informants appeared, either in the top left or top right corner of the screen (see Figure 1 I.). The informant addressed the child by saying "Oh, look!" ("Oh, guck mal!"). She then turned to point at the object, saying "This is a X, a X!" ("Das ist ein X, ein X!"). The speaker always appeared on the same side (the brunette actress always on the right, the blonde one always on the left). 


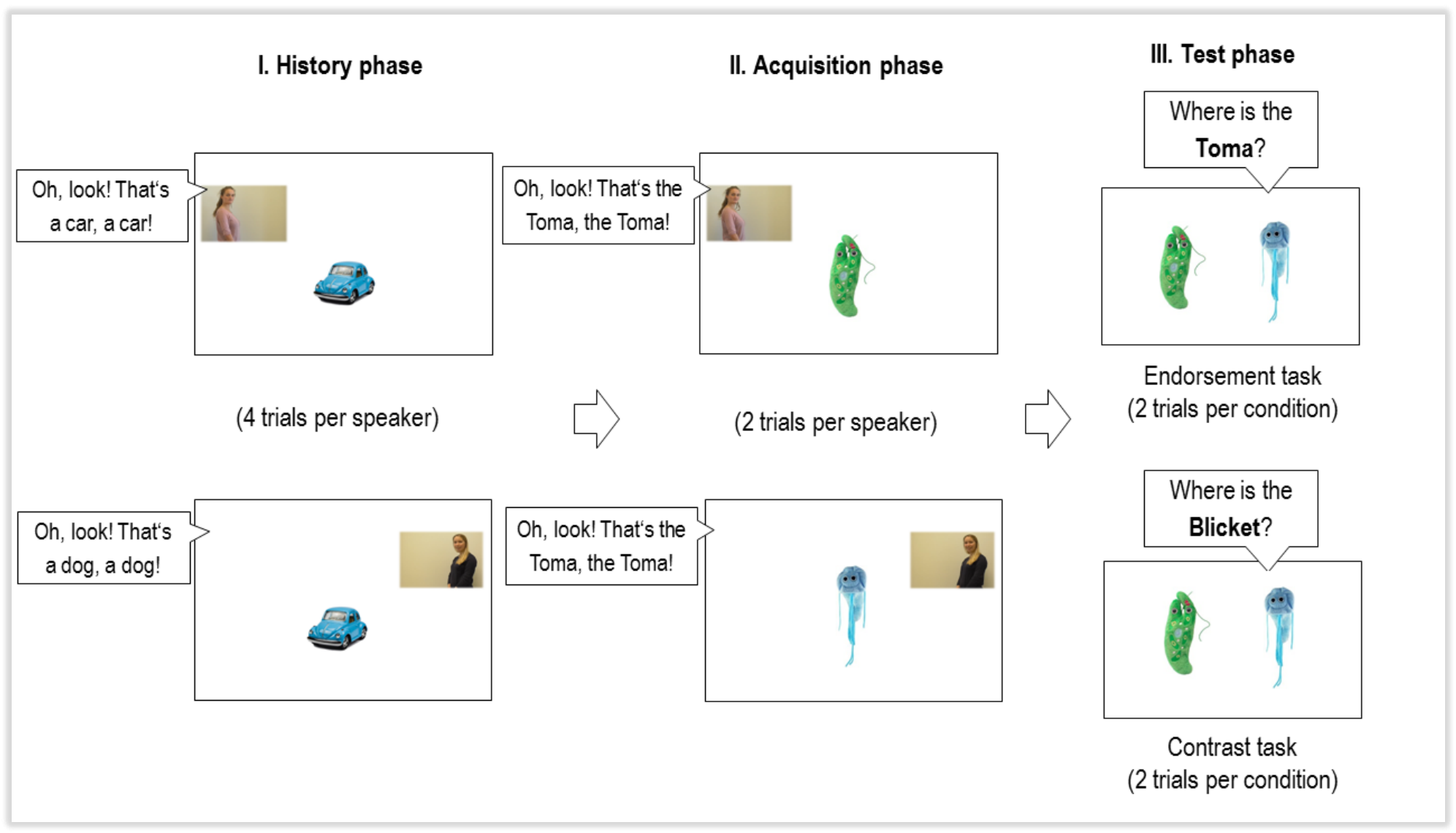

Figure 1. Schematic representation of the procedure in Study 1a; I) History phase; II) Acquisition phase; III) Test phase.

\section{Acquisition phase}

In the Acquisition phase of Study 1a, the informants proceeded to provide contradictory novel testimony, that is to teach the infant the same novel label, but to refer to different novel objects. To teach the children the novel labels, they saw four videos (two with each informant) with each video lasting 10.5 seconds. The clips were presented in blocks, two clips subsequently with the same speaker 
followed by two clips with the other speaker, with the order of which speaker went first counterbalanced across participants.

As labels in my experiments, I used 2-syllabic pseudowords following the structure "consonant-vowel-consonant-vowel", "Toma" here in Study 1a. As novel objects, I used the toy plush toys (a green plush toy, representing Euglena, and a blue plush toy, representing Giardia) that the child had encountered during the warm-up. Which novel object the reliable and unreliable speaker referred to was counterbalanced across children.

At the start of each film, an unfamiliar object appeared in the bottom middle of the screen. Then one of the informants appeared on the screen (in the same top corner as in the previous phase). She addressed the child (saying "Oh, guck mal!" - "Oh, look!" in her first labelling clip and "Schau mal!" - "Look!" in her second clip) and then turned to point at the object, providing the novel label for the novel object "This is the Toma, the Toma!" ("Das ist das Toma, das Toma!”) (see Figure 1 II.).

Following the History and Acquisition phase, children saw one additional trial of the History phase with each informant and then one additional trial of the Acquisition phase with each informant, to serve as a reminder.

\section{Eye-tracking test phase}

Each child participated in two eye-tracking tasks, first the Endorsement task and then the Contrast task (consisting of blocks of two trials per task). In both tasks the 
two novel objects, which the speakers had labelled in the Acquisition phase, appeared on screen and children heard a male voice asking for one of them (see Figure 1 III). In the Endorsement task, the male voice asked for the "Toma" (i.e., the novel label that had been taught by both of the two informants), whereas in the Contrast task, he used another completely novel two-syllable word, and asked for the "Blicket". I conducted two trials of each task with each child. These two trials differed slightly in phrasing of the question asked by the male voice: on one trial, the children heard the male voice saying "Where is the Toma, the Toma?" ("Wo ist das Toma, das Toma?"), and on the other trial "Can you see the Toma, the Toma?" (“Siehst du das Toma, das Toma?”).

Before each trial of the Endorsement task and the Contrast task, a fixation-cross appeared to guide the children's gaze to the center of the screen. The objects were presented on the left and right side of the screen, symmetrical to the center of the screen. The images of each object measured $344 \times 696$ pixels. The location of the objects was counterbalanced across the two trials of each task. The recording of the male voice was timed such that the objects were visible on screen for a preview time of $2.5 \mathrm{~s}$ before label onset. Total duration of these videos was $5.5 \mathrm{~s}$. Trials with two familiar objects and the male voice asking for one of the two objects were interspersed before each test trial. 


\section{Interactive test phase}

After completion of the eye-tracking part of the experiment, E1 and the child moved to a table in an adjacent room where the Interactive Task was conducted. In this task, the children were instructed to select one of two objects from a tray and put it on a chute behind them. After one trial of demonstration of how the chute works with a familiar object, two more trials with familiar objects were conducted with the child. On each of those trials, the child had to select a familiar target object among a familiar distractor. Following this familiarization with the procedure, two test trials with the plush toys, which had been labelled in the Acquisition phase, were conducted. Both objects were presented to the children on a tray in counterbalanced order. In the first test trial, E1 asked the child to put the "Toma" on the chute, in the second the "Blicket". Between the two test trials, another trial with a familiar object was interspersed.

As a manipulation check, E1 showed the child a printout of a screenshot of the two speakers and asked the children whom they thought was better at naming things. $91,4 \%$ of the 35 participants who gave an answer passed this manipulation check. Then the child and E2 played with the chute and the toys (except the two objects labelled by the two informants) for 4 minutes. After that time had passed, E1 asked the children whether they could recall the label for the blue object or the label for the green object (Free recall). 


\subsubsection{Analysis}

\section{Eye-tracking tasks}

I conducted both a time window analysis where I compared children's target fixations before and after label onset and a bootstrapped cluster-based permutation analysis of the whole time course. I defined areas of interest (AOI) for both locations left and right the center of the screen, where the images appeared during this task, as the size of the image plus $2.5 \%$ of the screen dimensions as overlap towards each side. Given an image size of 344 x 696 pixels and screen dimensions of $1920 \times 1080$ pixels, the dimensions of each AOI were defined as $440 \times 750$ pixels. There was no spatial overlap between the two AOIs. With a sampling rate of $60 \mathrm{~Hz}$, the eye-tracker provided the $\mathrm{x}$ - and $\mathrm{y}$-coordinates of participants' gaze direction for every $16 \mathrm{~ms}$ for both eyes individually. These coordinates were averaged across both eyes, unless the coordinates for one eye were missing (e.g., when due to participants' movements only one eye was detected by the eye-tracker). These gaze coordinates were then compared to the two AOIs, such that each data point was coded for whether the children looked at the target image or the distractor image, or anywhere else. In order to remove trials where children did not pay attention to the video presentation, I excluded trials in which children did not look at the screen for at least half of the trial's duration. This led to the exclusion of two trials of the Familiar-object condition, two trials of the Endorsement task and one trial of the Contrast task. 
Gaze data was aggregated across two time windows. The pre-naming window was a baseline window that counted all fixations 2000ms before label onset (500ms - 2500ms after video onset). The second window, the target window, counted all fixations from $240 \mathrm{~ms}$ after label onset (to adjust for reaction time and processing) to $2240 \mathrm{~ms}$ after label onset (2740ms $-4740 \mathrm{~ms}$ after video onset). For both windows, we determined the time that infants spent looking at the target or at the distractor. To calculate the proportion of target looking, the time infants spent looking at the target was divided by the total looking time at both target and distractor and averaged across the two trials of each task. The target in the familiar trials was, of course, the object to which the uttered label referred, and in both the Endorsement and the Contrast task, the object labelled by reliable was denoted as target. Hence, the object labelled by the unreliable speaker was denoted as distractor object in the Endorsement and Contrast task.

As this analysis relies on artificial time windows, I also computed a bootstrapped cluster-based permutation analysis (von Holzen \& Mani, 2012) to model infants' gaze behavior across the time course of each trial more directly. This analysis takes a summed statistic for each cluster of time bins that pass the .05 level of significance, and compares each to a "null" distribution of sum statistics obtained by bootstrap resampling data. To calculate the time course analysis, I used the eyetrackingR package (Dink \& Ferguson, 2018). The resampling rate was 1000 samples. 


\section{Interactive tasks}

As dependent measure for the Interactive Tasks, I counted children's choices, which object they selected as the one referred to by the label used by both informants and which one they selected as the one referred to by the novel label. I calculated binomial test to compare children's object choice to chance.

\subsubsection{Results}

\section{Eye-tracking tasks}

I predicted that, if children considered the speakers' previous accuracy, they should show the following response pattern: In the Endorsement task, when they heard the trained novel label "Toma", they should look at the target object, i.e., the one the reliable speaker had labelled. In the Contrast task, when they heard the completely novel label "Blicket", they should look at the object labelled by the unreliable speaker, i.e. the distractor.

To test this, I first looked at children's preferential looking time towards the target during the baseline window before label onset compared to during the target window after label onset across both tasks and the familiar object trials. I calculated a $3 \times 2$ ANOVA with proportion of target looking as dependent variable, and time window (pre-naming vs. post-naming) and type of task (Familiar, Endorsement, or Contrast) as within-subject factors. I found a main effect of type of task $\left(F(2,70)=15.11, p<.001, \eta_{p^{2}}=.30\right)$, a main effect of time 
window $\left(F(1,35)=25.1, p<.001, \eta_{p}^{2}=.42\right)$, and a significant interaction effect of time window and type of $\operatorname{task}\left(F(2,70)=14.05, p<.001, \eta_{p^{2}}=.29\right)$ (see Figure 2$)$.

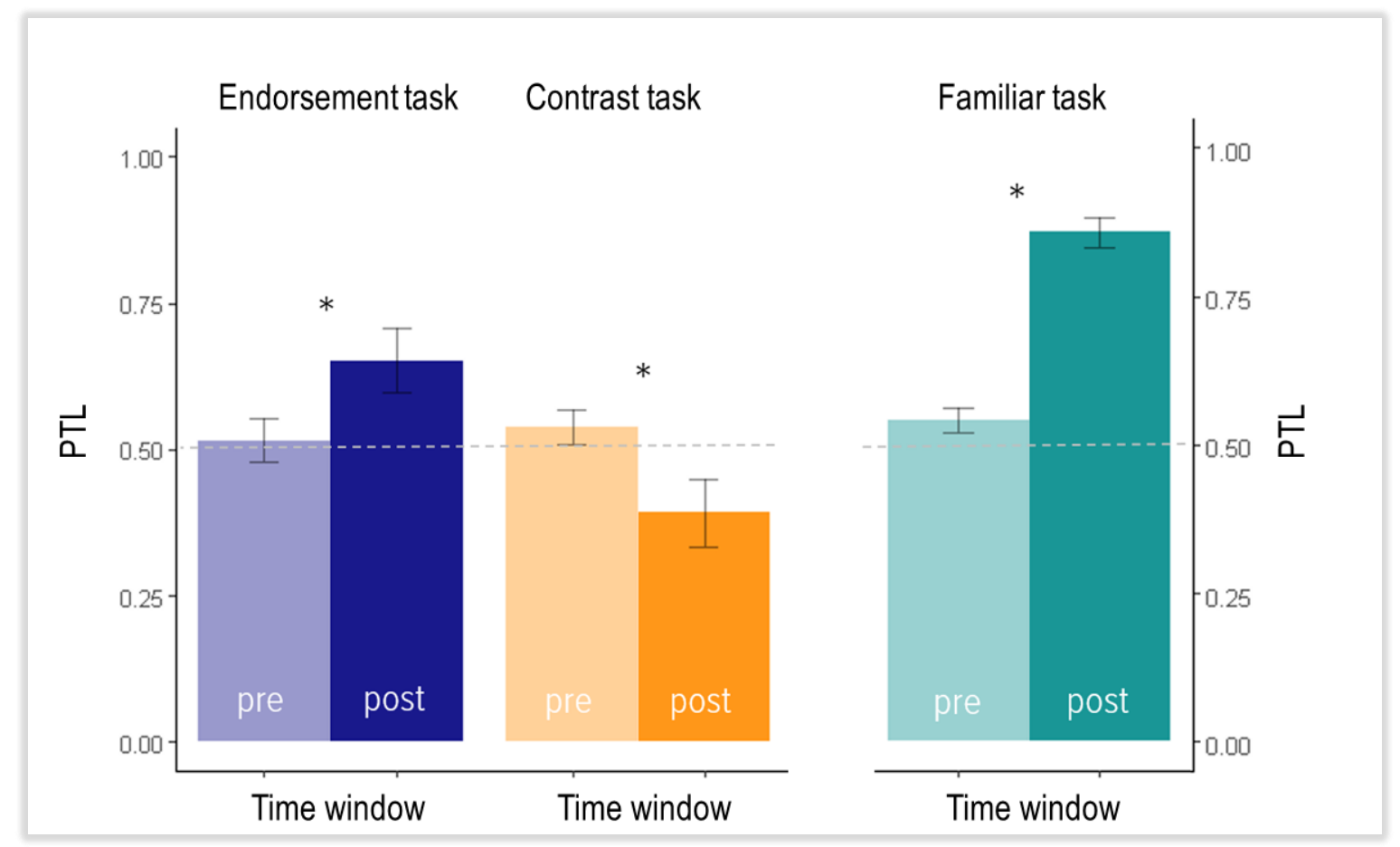

Figure 2. Proportion of target looking (PTL) in the Endorsement and Contrast task/ in response to the familiar label (target $=$ object labelled by reliable/familiar object). PTL calculated as time spent looking at the target object divided by the time spent looking at target plus at distractor. Time windows in relation to label onset (pre- label onset/ postlabel onset). Horizontal dotted line represents chance level.

As the children had no problems identifying the familiar objects, I then removed this task from the analysis and compared children's performance in the Endorsement and Contrast tasks directly. I calculated a 2x2 ANOVA with 
proportion of target looking as dependent variable, and time window (prenaming vs. post-naming) and type of task (Endorsement vs. Contrast) as withinsubject factors. I found a significant interaction effect of time window and type of task $\left(F(1,35)=5.07, p=.03, \eta_{p^{2}}=.13\right)$, a main effect of type task $(F(1,35)=4.14$, $\left.p=.049, \eta_{p}{ }^{2}=.106\right)$ and no main effect of time window $(F(1,35)=0.00, p=.983)$. Paired t-tests revealed a significant difference in target fixations across the preand post-naming phase in the Endorsement task $(t(35)=2.15, p=.039$, two-sided, $d=0.50$ ) while this difference was beyond our threshold for significance in the Contrast task $(t(35)=-1.93, p=.062)$. As predicted, children looked significantly more towards the object labelled by the reliable speaker (target) than the object labelled by the unreliable speaker (distractor) after they heard the label used by both informants (Endorsement task) compared to after they heard the completely novel label (Contrast task). No other post hoc comparison was significant.

The time course analysis presented a concurring picture (see Figure 3). Children's proportion of target looking in the Endorsement and Contrast tasks deviated significantly from each other between $1050 \mathrm{~ms}$ after label onset to 2200 ms after label onset (cluster $t$ statistic $=-62.13$, Monte Carlo $p=.01$ ) (see grayed out area in Figure 3). Ten of the 1000 random partitions resulted in a clusterlevel statistic that was larger in absolute value. 


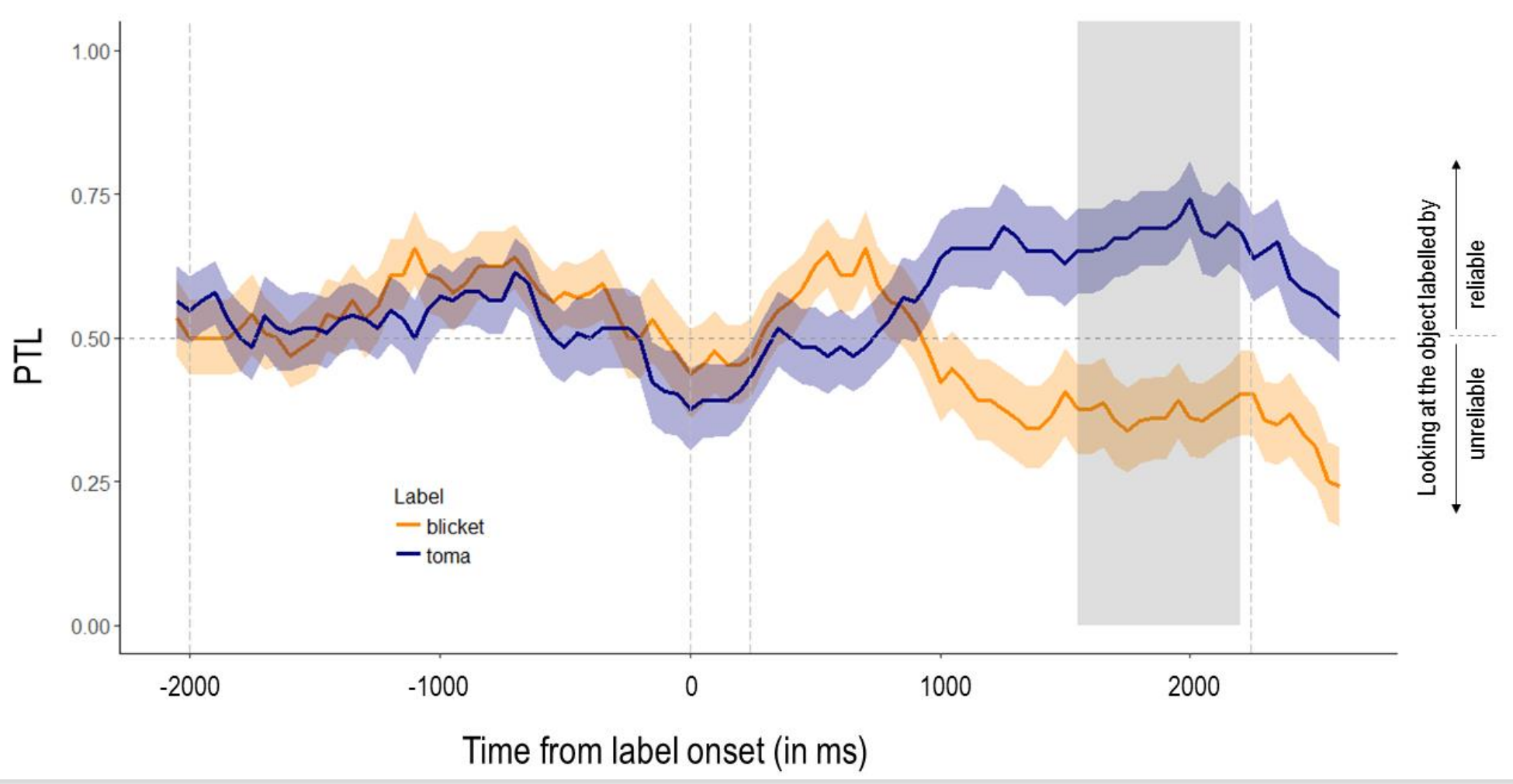

Figure 3. Proportion of target looking (PTL) across the time course in the eye-tracking Endorsement (blue line) and Contrast (orange line) tasks. Grayed out area represents cluster of significant difference. Vertical dotted lines represent time windows used for time window analysis (pre-naming window: -2000 to $0 \mathrm{~ms}$ from label onset; post-naming window: 240 to $2240 \mathrm{~ms}$ ), horizontal dotted line represents chance level.

\section{Interactive tasks}

Children also showed the same pattern of responses in the interactive versions of these tasks (see Figure 4). In the interactive Endorsement task, when asked for the "Toma" (i.e., the label trained by both speakers), children selected the object labelled by the previously accurate informant at rates above chance $(72,2 \%$, 
exact binomial test, $p=.006)$. In contrast, when children heard the completely novel label "Blicket" children selected the object labelled by the unreliable informant significantly more often than predicted by chance $(69,4 \%$, exact binomial test, $p=.014)$.

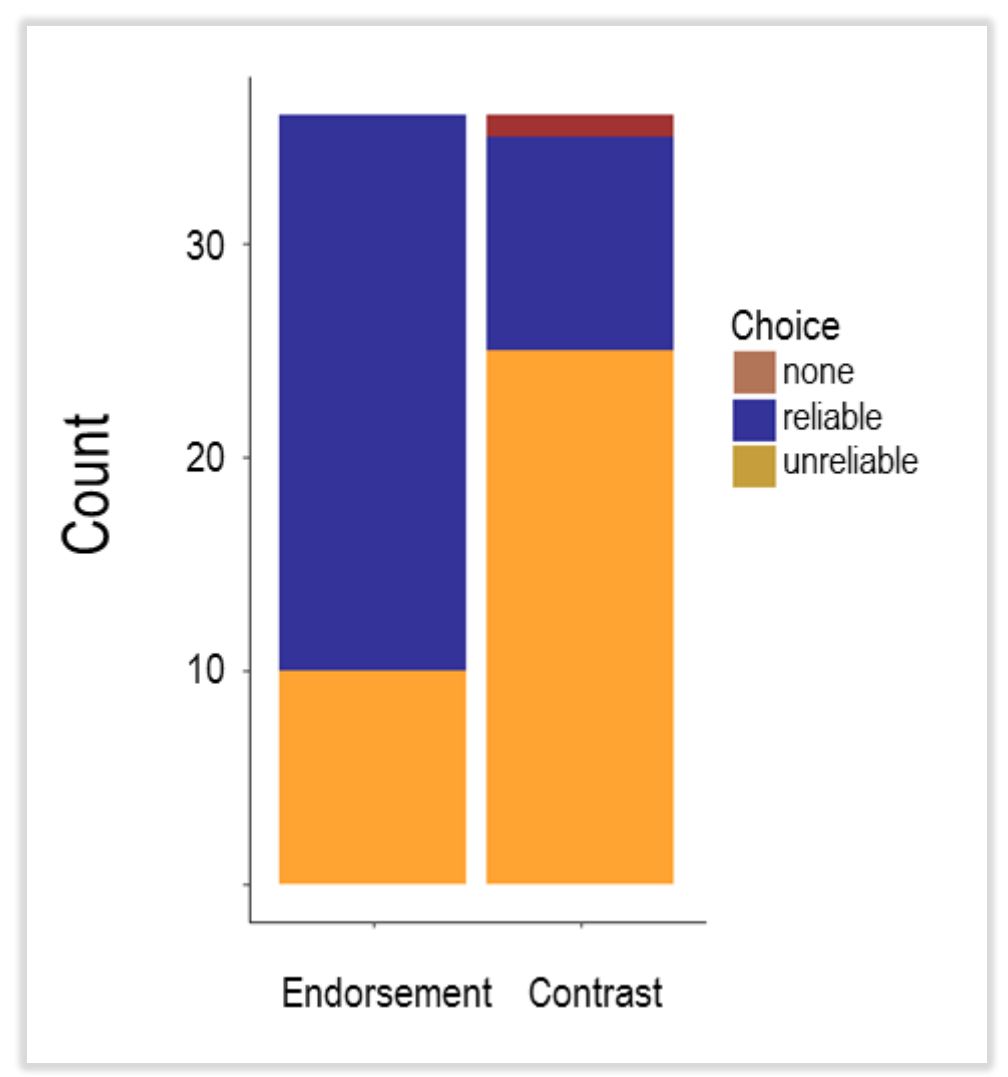

Figure 4. Children's object choices in the interactive Endorsement and Contrast tasks.

\section{Correlation between eye-tracking and interactive measure}

In order to validate eye-tracking as method to explore children's trust in testimony, I explored the correlation between children's performance in the eyetracking version and the interactive version of each task. For the Endorsement 
tasks, I found that the proportion of target looking in the eye-tracking version of the task was significantly correlated with children's target choice in the interactive Endorsement task (point-biserial correlation, $r=.58, p<.001$ ). That is, the higher the proportion of looking towards the object labelled by the reliable speaker in the eye-tracking Endorsement task, the more likely children were to choose this object in the interactive Endorsement task. Similarly, the proportion of target looking during the Contrast task was significantly correlated with children's choice in the interactive Contrast task $(r=.48, p=.003)$. The higher the proportion of looking towards the unreliable object in the eye-tracking Contrast task, the more likely children were to choose the unreliable object in the interactive Contrast task.

\section{Free recall}

The free recall proved to be rather difficult for our participants. Only eight children remember the label "Toma", ten others uttered a label that was similar (e.g. “Tomfa”, “Toa”, “Tuba”, “Puma”, “Oma”), and of those 18, 13 used it for the object that had been labelled by reliable, four used it for the object that had been labelled by unreliable, and one told us that both objects are called Toma. 


\subsubsection{Discussion}

In the interactive tasks, children endorsed the object labelled by the reliable informant over the object labelled by unreliable as referent of the label used by both. Children also expected a novel label to refer to the object labelled by the unreliable speaker rather than the object labelled by the reliable speaker. When the information provided by the differently reliable sources was conflicting during the acquisition, children disregarded object-label mappings offered by the unreliable speaker to a degree such that additional information by a naïve informant is preferably accepted. This replicates the findings by many studies (e.g. Birch et al., 2008; Koenig et al., 2004; Koenig \& Harris, 2005; Scofield \& Behrend, 2008), that children prefer information from reliable speakers over unreliable speakers when the testimony was conflicting during the acquisition. This shows that children can use (in-)accuracy information from prior interactions to adjust their reliance on novel testimony from different sources. The findings in our eye-tracking tasks suggest that these processes already influence children's spontaneous gaze behavior. Upon hearing the label used by both informants, children looked more towards the object labelled by the reliable speaker compared to the object labelled by the unreliable speaker. When hearing a novel label instead, children shifted their gaze towards the object labelled by the unreliable speaker. This suggests that eye-tracking can be used to investigate selective social learning. The correlation we found between the eye-tracking tasks and the interactive tasks further support this conclusion. 
Considering the findings of this study, we can conclude that when a reliable and unreliable informant offer information that is in conflict with each other, children will endorse information from the reliable informant over information from the unreliable informant. They will even discount information by the unreliable informant in favor of novel information by a third person with unrevealed accuracy. However, these finding do not distinguish between the proposed mechanisms for children's selectivity. Given the contradiction in testimony during the Acquisition phase and the forced-choice task, children are not directly tested for the information provided by the unreliable informant. We know that children treat information from a reliable informant preferably, but we do not know whether they attend to information from an unreliable informant differently, i.e. only encode episodic information about the labeling event, or whether they do not attend to the unreliable informant at all. Further, we cannot tell whether this selectivity is necessarily based on the contradiction between testimonies by different sources when this information is encoded, i.e. whether the conflicting testimony triggers children's selective encoding of such testimony.

To investigate whether we can generally find differences in how children encode information from accurate and inaccurate sources, I provided them with compatible information from a reliable and an unreliable source during the Acquisition phase in Study $1 \mathrm{~b}$ and with tasks with varying difficulty and implications for the proposed mechanisms. 


\subsection{Study $\mathbf{1 b}$}

In my second study, I was interested in whether children generally encode information from a reliable and an unreliable informant differently. To investigate that, I provided children with compatible information from a previously accurate and a previously inaccurate source. In two eye-tracking and one interactive tasks, I examined the extent to which children attended to and learned from the two sources.

In Study $1 b$, children first saw a video presentation of two female informants labelling familiar objects accurately or inaccurately, just like in Study 1a. The main difference to Study 1a was that in the Acquisition phase both informants provided two different novel labels for two different objects, i.e. information that cannot be understood as in conflict with each other. In the eye-tracking Recognition task, children saw four objects on the screen - the two objects labelled by the reliable and the unreliable informant, a familiar object and an unfamiliar, unlabeled object - and heard the label used by one of the two informants. If we assume that children's selectivity is usually caused by inhibiting the acquisition of any "bad" information, as suggested by the Blocking account, we would expect to find a difference in performance in this task based on the informant's reliability. In similar preferential looking paradigms, contingency learning was enough to elicit gaze upon hearing a label towards the object that was referred to by that label (Bannard \& Tomasello, 2012). Thus children should be selective in this task, only 
if the inhibition of information by unreliable obstructed the formation of any associations between the label used by unreliable and the object labelled by her. In the second task in Study $1 \mathrm{~b}$, the eye-tracking Disambiguation task, children were confronted with one of the two previously labelled unfamiliar objects and a completely novel object, and heard a male voice ask for the referent of a novel label. Usually, when children see a familiar and a novel object, and someone refers to one of the two objects with a novel label, children infer that the novel label refers to the novel object (Jaswal, 2010b; Merriman \& Schuster, 1991). This is called "disambiguation effect". As we assume children to generally learn from an accurate informant, we expect children to easily be able to infer the referent of the novel label when the novel object was juxtaposed to the object labelled by the reliable informant. Whether children similarly rely on information from the previously inaccurate informant will help further discern the proposed mechanisms of children's selective trust. If children inhibited any information from an unreliable source, the Blocking account would assume children to not treat a novel object and an object that had been labelled by the unreliable informant differently. Rather, children should look at the two objects and assume that both of them appear equally novel. Performance in response to hearing the novel label should be at chance. Following the Semantic gating account, children should not assume that the unreliable object already has a label, but they should know that it had been labelled before. Presumably, this could be enough to solve the Disambiguation task when the novel object is juxtaposed to the object labelled by the inaccurate informant. 
Finally, the third task in this study consisted of an interactive version of the Recognition task. Children had to pick out of four physical representations of the objects the one of which they thought a label referred to. Only if a strong enough semantic link was established between the object labelled by unreliable and the label she used, should children be able to solve this task. If we assume only weak semantic links for information from an unreliable source, as suggested by the Semantic gating account, or no links at all, as suggested by the Blocking account, children should only be able to solve this task based on the object-label mapping they learned from the previously accurate informant, not from the previously inaccurate informant.

\subsubsection{Method}

\subsubsection{Participants}

Thirty-five monolingual German children $(\mathrm{M}$ age $=65.6$ months, range $=60.3-71.9$ months, 18 female) were included in the final sample of this experiment. Participants were recruited from the same database as children in Study 1a. None of the participants had known visual or auditory impairments. Parents gave informed consent for participation of their child. Eight additional children participated but could not be included in the final sample because of technical difficulties that led to the eye-tracking device not recording any gaze data. Two children stopped participating in the middle of the experiment and could thus not be included in the final sample. 


\subsubsection{Set up}

For the eye-tracking tasks, we used an almost identical setup as in Study 1a, except a different model of eye-tracker, a SMI Red-n eye-tracker. This eye-tracker also records gaze data at $60 \mathrm{~Hz}$ with an average accuracy of $0.4^{\circ}$ visual angle. It was attached to the same monitor as in Study 1a with a display resolution of $1920 \mathrm{x}$ 1080 pixels. During the experiment, the children sat on the adjustable chair that we used to ensure that children's eyes were inside the detectable range of the eyetracker. The calibration and validation procedure was identical to Study 1a. EPrime 2.0 was used as presentation software. The novel objects that children handled, and saw still pictures of, were the same plush toys as in Study 1a representing microbes (the green plush toy, representing Euglena, and the blue plush toy, representing Giardia).

\subsubsection{Procedure}

As in Study 1a, the session started with a warm-up phase, in which the child played with the two experimenters (E1 and. E2). During this phase, the child was allowed to play for up to 20 s with either plush toy that would later be named by the reliable and unreliable informants. Then for the eye-tracking part of the study, the child was seated in front of the screen, to which the eye-tracker was attached, and the seat's height and distance to the screen were adjusted to ensure that 
children's eyes were inside the detectable range of the eye-tracker to record children's gaze.

Subsequently, each test session consisted of the following phases. First, infants were introduced to both informants and their labelling accuracy in the History phase. In the Acquisition phase, both informant proceeded to provide novel testimony, that is a novel label for a novel object. In our second study, the information provided by the two informants was not contradictory, but instead compatible, i.e. the two informants offered two different novel labels for the two different novel objects. Children's appreciation of testimony from the two sources was then investigated in two eye-tracking tasks: the Recognition task and the Disambiguation task. Finally, as in Study 1a, infants participated in interactive versions of these tasks. In the very beginning of the experiment and as a transition scene after the History phase and after the Acquisition phase, the same attention getter video of balloons to piano music as in Study 1a was included in this study.

\section{History phase}

At the beginning of the video presentation, the children saw a 10s greeting part to introduce the two informants, just as in Study 1a. To familiarize the children with the accuracy of the two informants, they saw the same 8 video clips (4 with each informant) as in Study 1a, where the actresses labelled familiar objects (apple, baby, car, dog). Children first saw one of the informants label three objects in turn, then the other informant labelled those same three objects in reversed order, followed 
by each labelling a fourth object. If the speaker was reliable, she used the correct label to refer to the objects visible on screen. If she was unreliable the label she used did not refer to the object present on screen, but was from the pool of familiar labels used by the reliable informant during the experiment. The identity of the reliable informant and order of the informants was counterbalanced across infants. Each of the film clips of the History phase lasted 10s.

\section{Acquisition phase}

The label training in Study $1 b$ was very similar as in Study 1a. The main difference was that the informants now provided compatible novel testimony instead of contradictory testimony. To teach the children the novel labels, they saw four videos (two with each informant) where the informants provided labels for the novel objects in the same manner as in Study 1a, with each video lasting $16 \mathrm{~s}$ seconds. As labels, 2-syllabic pseudowords were used, Toma and Schufi (Shoofi). The novel objects were the same we used in Study 1a (Euglena (green) and Giardia (blue)), and in the warm up before the History phase of Study 1b. Which label the reliable speaker used and which novel object she referred to was counterbalanced across children.

\section{Eye-tracking test phase}

Each child participated in two eye-tracking tasks, first the Recognition task and then the Disambiguation task. 
In the Recognition task, four objects appeared on screen: the two objects labelled by the reliable and the unreliable informant, a familiar object (a ball) and an unfamiliar, unlabeled object (another microbe plush toy, Herpes (yellow)). The image of each object measured $576 \times 324$ pixels. The objects appeared in the four corners of the screen (see Figure 5 III.), with their locations randomized among the four possible locations. In six trials of this task, a male voice asked for the location of the referent of the label that the previously accurate informant had used (reliable object), for the location of the referent of the label that the previously inaccurate informant had used (unreliable object), or for the location of the familiar object. We conducted two trials for each target object (reliable object, unreliable object, familiar object) task with each child. Just like in Study 1a, each of the two trials per target differed slightly in phrasing of the question asked by the male voice ("Where is the $X$, the $X$ ?" - "Wo ist das $X$, das X?" versus "Can you see the $X$, the $X$ ?" “Siehst du das X, das X?"). Before each trial of the Recognition task, a fixation-cross appeared to guide the children's gaze to the center of the screen. The recording of the male voice was timed such that the objects were visible on screen for a preview time of $4 \mathrm{~s}$ before label onset. Total duration of these videos was $12 \mathrm{~s}$. 


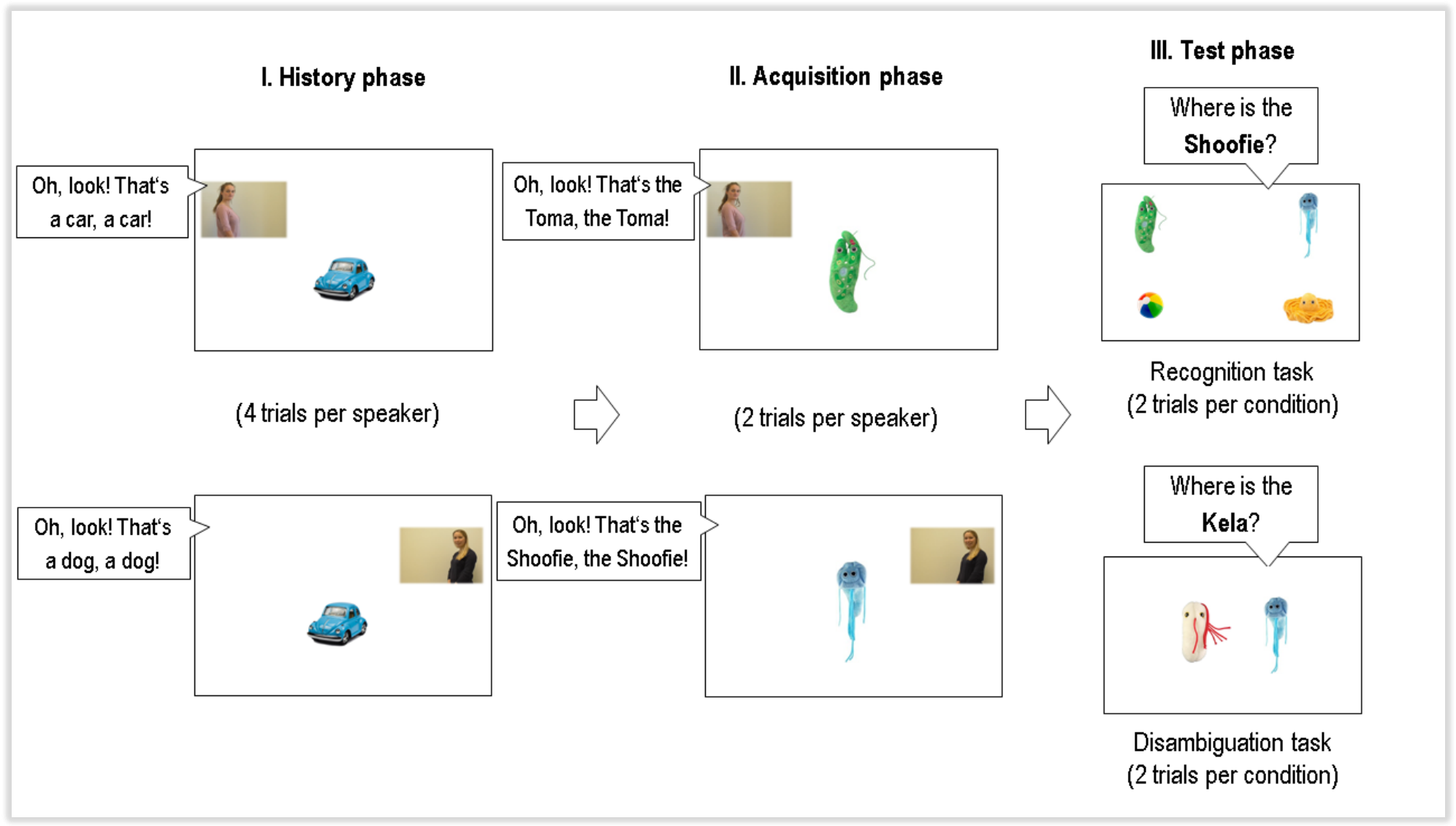

Figure 5. Schematic representation of the procedure in Study 1b; I) History phase; II) Acquisition phase; III) Test phase (here: unreliable condition).

In the Disambiguation task, two objects appeared on screen: one of the two objects labelled by the reliable or the unreliable informant, and an unfamiliar, unlabeled object (another microbe plush toy, either Orange or White; see Figure 5 III.). In four trials of this task, the reliable object ( 2 trials) or the unreliable object ( 2 trials) was presented juxtaposed to the novel object, that the children had not seen before and the male voice asked for the referent of a novel label, that the children had not heard before (either "Kela" or "Dagu"). The image of each object measured $565 \mathrm{x}$ 
340 pixels. The objects appeared on screen symmetrical to the center of the screen left and right. Location of the objects was counterbalanced across the two trials.

Just like in Study 1a and the Recognition task of Study 1b, each of the two trials per target differed slightly in phrasing of the question asked by the male voice ("Where is the $X$, the $X$ ?" - "Wo ist das $X$, das $X$ ?" versus "Can you see the $X$, the $X$ ?" “Siehst du das X, das X?"). Before each trial of the Disambiguation task, a fixationcross appeared to guide the children's gaze to the center of the screen. The recording of the male voice was timed such that the objects were visible on screen for a preview time of $2.5 \mathrm{~s}$ before label onset. Total duration of these videos was $8 \mathrm{~s}$.

\section{Interactive task}

After completion of the eye-tracking part, E1 and the child moved to a table in an adjacent room where the interactive Recognition task was conducted. In this task, the children were instructed to select one of the four objects, which had been presented to the child in the eye-tracking Recognition task, from a tray and put it in a box in front of them. In three trials, E1 asked the child first to put the familiar object (ball) in the box, then the referent of the label that the accurate informant had used, and finally the referent of the label that the inaccurate informant had used. The four objects were presented to the children on the tray in randomized order. Which speaker's label was asked for first was counterbalanced across children. After the completion of this task, as a manipulation check, E1 showed the child a printout of a screenshot of the two speakers and asked the children whom 
they thought was better at naming things. $89.3 \%$ of the 28 participants who gave an answer passed this manipulation check.

\subsubsection{Analysis}

\section{Eye-tracking tasks}

I conducted both a time window analysis where we compared children's target fixations before and after label onset and a bootstrapped cluster-based permutation analysis of the whole time course, analogous to our analysis in Study 1a. I defined areas of interest (AOI) for each of the four location in the corners of the screen where the images appeared during the Recognition task and the AOIs for each of the two locations left and right the center of the screen where the images appeared during the Disambiguation task as the size of the image plus $2.5 \%$ of the screen dimensions as overlap towards each side. Given an image size of $576 \times 324$ pixels in the Recognition task and of 565 × 365 pixels in the Disambiguation task, the dimensions of each AOI were defined as $672 \times 378$ pixels and as $661 \times 420$ pixels respectively. There was no spatial overlap between any of the AOIs. The $\mathrm{x}$ - and $\mathrm{y}$ coordinates of participants' gaze direction provided by the eye-tracker were averaged across both eyes, unless the coordinates for one eye were missing. These gaze coordinates were then compared to the four AOIs (two in the Disambiguation task) and coded for whether the children looked at the target image or at any of the distractor images, or anywhere else. In order to remove trials where children did not pay attention to the video presentation, I excluded trials in which children 
did not look at the screen for at least half of the trials duration. This led to the exclusion of five trials of the unreliable condition of the Recognition task, and eleven trials of the Disambiguation task, two of the unreliable condition and nine of the reliable condition. The relatively high amount of dropped trials was likely due to unfavorable lighting conditions at the lab during the time when this study was conducted.

Gaze data was aggregated across two time windows. The first window was a baseline window that counted all fixations $2000 \mathrm{~ms}$ before label onset $(2000 \mathrm{~ms}-$ 4000ms after video onset for the Recognition task, 500ms - 2500ms after video onset for the Disambiguation task). The second window, the target window, counted all fixations from $240 \mathrm{~ms}$ after label onset, to adjust for reaction time and processing, to $2240 \mathrm{~ms}$ after label onset $(4240 \mathrm{~ms}-6240 \mathrm{~ms}$ after video onset for the Recognition task, 2740ms - 4740ms after video onset for the Disambiguation task). For both windows, we determined the time that infants spent looking at the target or at the distractor. To calculate the proportion of target looking, the time infants spent looking at the target was divided by the total looking time at both target and distractors and averaged across the two trials of each task.

In the Recognition task, the target in the familiar trials was the familiar object. In the trials when the male voice asked for the referent of the label used by the reliable informant, the object that the reliable speaker had labelled was denoted as the target. In the trials when the male voice asked for the referent of the label used by the unreliable informant, the object that the unreliable speaker had 
labelled was denoted as the target. The three other objects, which were not denoted as target, were denoted as distractors. In the Disambiguation task, the novel objects presented juxtaposed to the objects labelled by the reliable or unreliable speaker were denoted as target. The object labelled by reliable or unreliable was denoted as distractor.

As this analysis relies on artificial time windows, I also computed a bootstrapped cluster-based permutation analysis to model infants' gaze behavior across the time course of each trial more directly, in parallel to my analysis in Study 1. To calculate the time course analysis, I used the eyetrackingR package (Dink \& Ferguson, 2018). The resampling rate was 1000 samples.

\section{Interactive Recognition task}

As dependent measure for the interactive task, I counted infants' choices, which object they selected as the one referred to by the label used by both informants and which one they selected as the one referred to by the novel label. To assess whether children chose the target object significantly above chance, I used exact binominal tests and compared the number of correct trials to the total number of trials a child participated in, with chance set at $25 \%$, i.e., this more conservative analysis treated trials on which children did not choose as incorrect rather than excluding them. 


\subsubsection{Results}

\section{Recognition eye-tracking task}

I predicted that if children had learned the labels they heard the reliable and unreliable speaker use, they should shift their gaze towards the respective object upon hearing the label used by the male voice in the test trials. To test whether children looked towards the objects in response to hearing the labels, I first examined children's preferential looking time towards the target during the baseline window before label onset compared to during the target window after label onset, when hearing the familiar label, the label used by the reliable speaker or the label used by the unreliable speaker. I conducted a $3 \times 2$ repeated-measures ANOVA with proportion of target looking as dependent variable, and time window (pre-naming vs. post-naming) and target (familiar object, object labelled by reliable, object labelled by unreliable) as predictors. As they did not provide data points for every condition, one child had to be excluded from the analysis of the Recognition task. I found a significant main effect of target $(F(2,66)=9.18 ; p$ $\left.<.001, \eta_{p}{ }^{2}=.22\right)$, a significant main effect of window $\left(F(1,33)=109.4 ; p<.001, \eta_{p}^{2}\right.$ $=.77)$ and a significant interaction between target and window $(F(2,66)=22.46 ; p$ $\left.<.001, \eta_{p}{ }^{2}=.41\right)($ see Figure 6). 


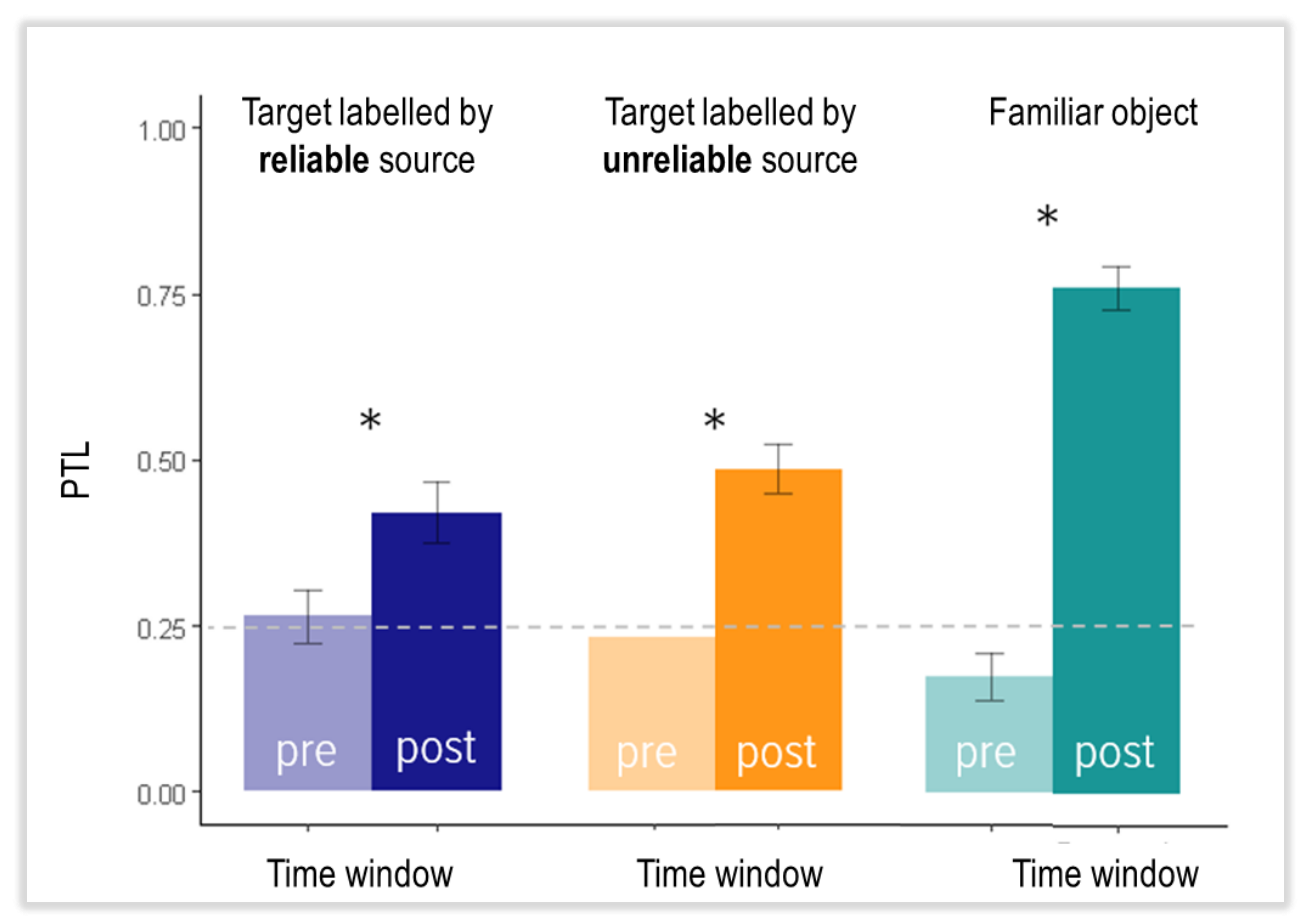

Figure 6. Proportion of target looking (PTL) in the Recognition task by condition (target object labelled by reliable, target object labelled by unreliable, target object familiar,). PTL calculated as time spent looking at the target object divided by the time spent looking at target plus at any of the distractors. Horizontal dotted line represents chance level.

As these effects are likely to be driven in large parts by children having little to no difficulties identifying a familiar object among three novel objects, I then omitted the trials where the familiar object was the target to compare children's performance based only on the information offered by the two informants. I conducted a $2 \times 2$ repeated-measures ANOVA with proportion of target looking as dependent variable, and time window (pre-naming vs. post-naming) and target (object labelled by reliable vs. or object labelled by unreliable) as predictors. 
There was a significant main effect of time window $\left(F(1,33)=24.14, p<.001, \eta_{p}{ }^{2}\right.$ $=.45)$. There was no main effect of target $(F(1,33)=0.01, p=.92)$, and no interaction between the two effects $(F(1,33)=0.53, p=.47)$. Paired $t$-tests revealed a significant difference in the pre- compared to the post-naming window for the object labelled by reliable $(t(34)=2.98, p=.005, d=.60)$, as well as for the object labelled by unreliable $(t(33)=4.72, p<.001, d=.93)$. Children looked proportionally more towards the target object in the post-naming window, i.e. after they heard the label, compared to during the pre-naming baseline window, indifferent of who had labelled that object before. Post-hoc comparison further showed that children's target looking both during the pre-naming $(t(33)=0.48, p=.63)$ or the post-naming window $(t(34)=-0.48, p=.63)$ did not differ whether the target had been labelled by reliable or unreliable.

\section{Disambiguation eye-tracking task}

I predicted, that if children had used the labels they heard the reliable and unreliable speaker use to disambiguate the meaning of a novel label, they should shift their gaze towards the novel object upon hearing the label used by the male voice in the test trials. To test whether children looked towards the novel objects in response to hearing the novel labels, I examined children's preferential looking time towards the target during the baseline window before label onset compared to during the target window after label onset, when the novel object was juxtaposed to the object labelled by the reliable speaker or the object labelled by 
the unreliable speaker. I conducted a 2x2 repeated-measures ANOVA with proportion of target looking as dependent variable, and time window (pre-naming vs. post-naming) and distractor (object labelled by reliable vs. object labelled by unreliable) as predictors. As they did not provide data points for every condition, two children had to be excluded from the analysis of the Disambiguation task. There was a significant main effect of time window $\left(F(1,31)=60.64, p<.001, \eta_{p^{2}}\right.$ $=.66)$, there was no main effect of distractor $(F(1,31)=0.01, p=.93)$, and no interaction between the two effects $(F(1,31)=0.25, p=.62)$ (see Figure 7 ).

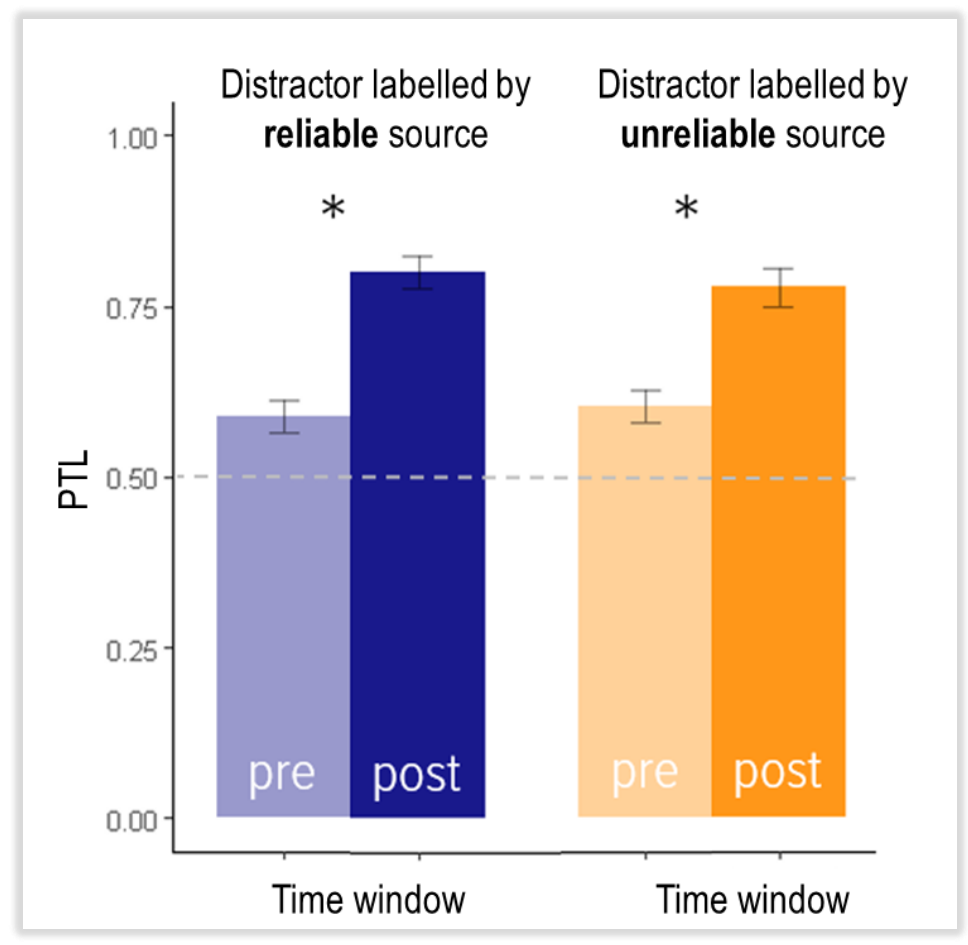

Figure 7. Proportion of target looking (PTL) in the Disambiguation task by condition (distractor labelled by reliable, distractor labelled by unreliable). PTL calculated as time spent looking at the target object divided by the time spent looking at target plus at any of the distractors. Horizontal dotted line represents chance level. 
Paired t-tests revealed a significant difference in the pre- compared to the postnaming window for the object labelled by reliable $(t(32)=5.84, p<.001, d=1.45)$, as well as for the object labelled by unreliable $(t(33)=4.63, p<.001, d=1.04)$. Children looked proportionally more towards the novel object that was presented juxtaposed to a previously labelled distractor in the post-naming window, i.e. after they heard the novel label, compared to during the pre-naming baseline window, indifferent of who had labelled that distractor before. Post-hoc comparison further showed that children's target looking both during the pre-naming $(t(31)=-0.30, p$ $=.76)$ or the post-naming window $(t(31)=0.42, p=.67)$ did not differ whether the distractor had been labelled by reliable or unreliable.

\section{Time course analysis}

To investigate whether we find differences in the looking behavior across the whole time course depending on the prior accuracy of the speaker, I also computed a bootstrapped cluster-based permutation analysis (von Holzen \& Mani, 2012) to model children's gaze behavior more directly. This analysis showed that both across the whole time course of the Recognition task as well as across the whole time course of the Disambiguation task, there were no clusters of time bins that showed a significant difference in looking behavior between our conditions. Children's looking behavior was the same when the target in the Recognition task had been labelled by reliable or unreliable (see Figure 8). 


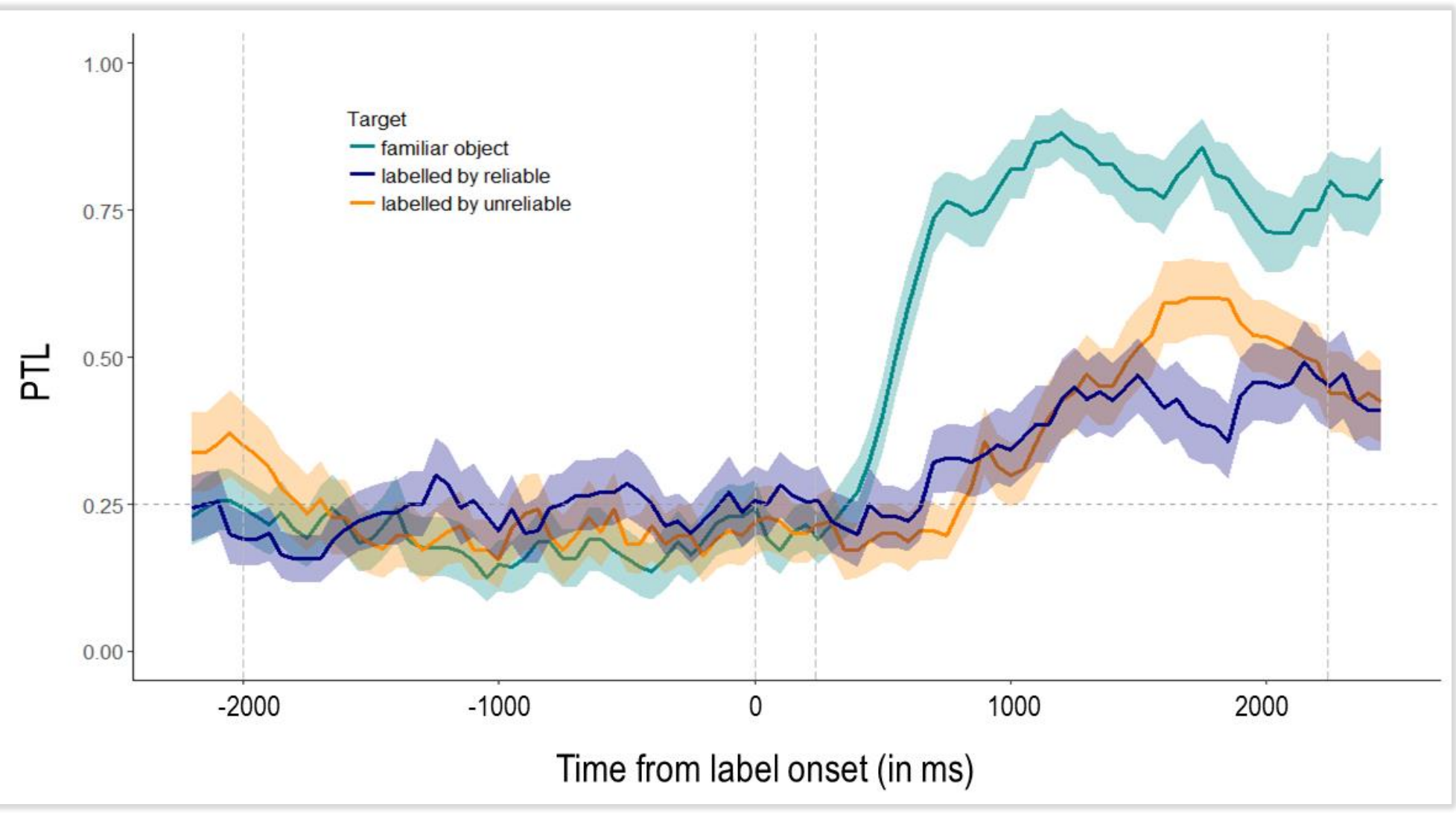

Figure 8. Time course of the proportion of target looking (PTL) in the Recognition task by condition (target object familiar, target labelled by reliable, target labelled by unreliable). PTL calculated as time spent looking at the target object divided by the time spent looking at target plus at any of the distractors during each $50 \mathrm{~ms}$ bin. Vertical dotted lines represent time windows used for time window analysis (pre-naming window: -2000 to $0 \mathrm{~ms}$ from label onset; post-naming window: 240 to $2240 \mathrm{~ms}$ ), horizontal dotted line represents chance level. 
Similarly, there was also no difference in looking behavior in the Disambiguation task when the distractor had been labelled by reliable or unreliable (see Figure 9).

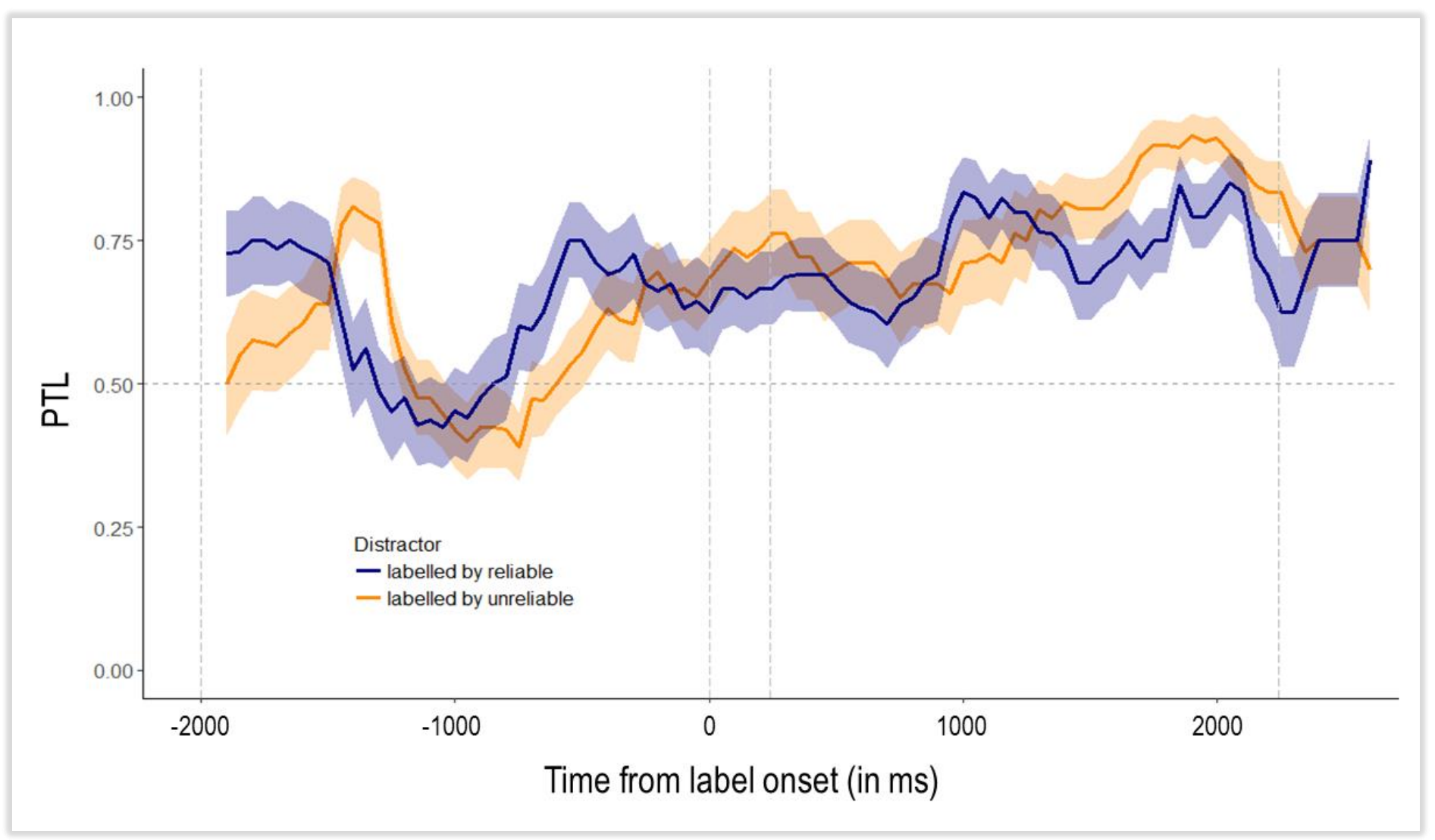

Figure 9. Time course of the proportion of target looking (PTL) in the Disambiguation task by condition (distractor labelled by reliable, distractor labelled by unreliable). PTL calculated as time spent looking at the target object divided by the time spent looking at target plus at any of the distractors during each $50 \mathrm{~ms}$ bin. Vertical dotted lines represent time windows used for time window analysis (pre-naming window: -2000 to $0 \mathrm{~ms}$ from label onset; post-naming window: 240 to $2240 \mathrm{~ms}$ ), horizontal dotted line represents chance level. 


\section{Interactive task}

I counted children's successes when selecting the object referred to by the label used by the reliable informant or by the unreliable informant. This was the dependent measure for the Interactive Task. Binomial tests revealed that children's choices differed significantly from chance both for the reliable condition $(p=.001)$ as well as for the unreliable condition $(p=.004)$ (see Figure 10).

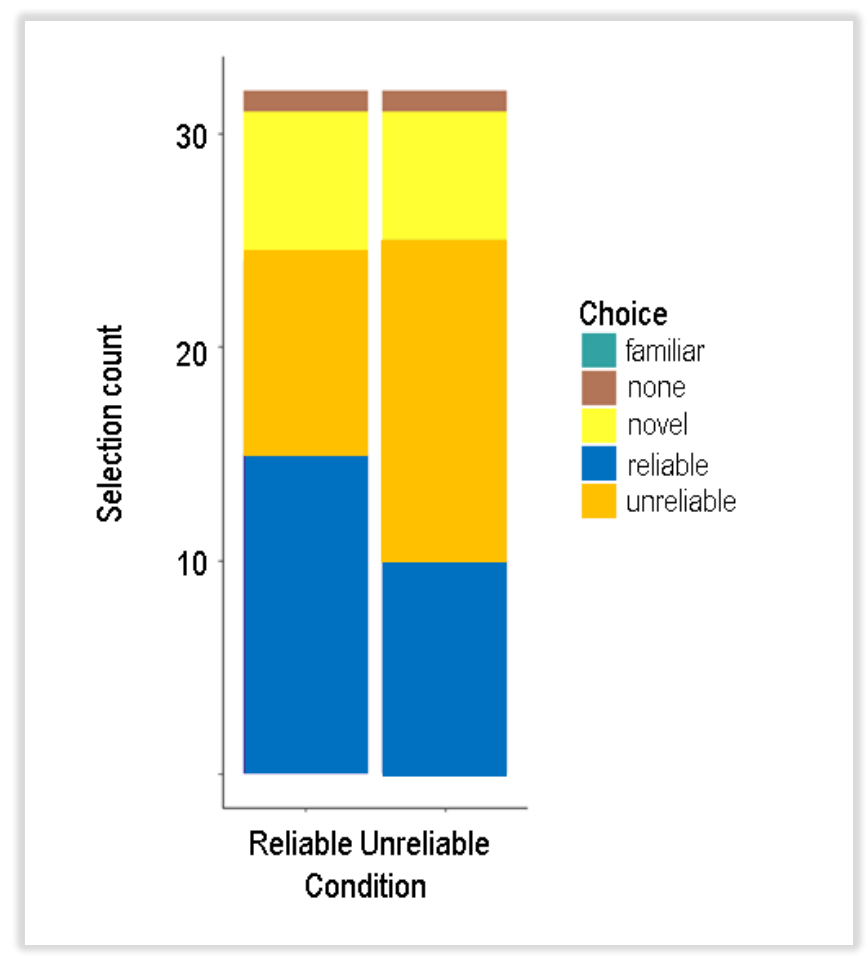

Figure 10. Children's object choice in the interactive task by condition (target object labelled by reliable, target object labelled by unreliable). 


\section{Correlation between eye-tracking and interactive measure}

I also calculated a point-biserial correlation between performance in the eyetracking tasks and whether children chose the target object during their interactive counterparts. I found that a higher proportion of target looking during the postnaming phase when the target had been labelled by the reliable informant did not correspond with a higher success rate in interactive trials where the target was the reliable object $(p=.766)$. Similarly, a higher proportion of target looking during the post-naming phase when the target had been labelled by the unreliable informant did not correspond with a higher success rate in interactive trials where the target was the unreliable object $(p=.127)$.

\subsubsection{Discussion}

Children were able to solve the Recognition eye-tracking task in Study $1 \mathrm{~b}$ based on information from both the reliable and the unreliable informant. In response to the familiar label, they looked towards the familiar object, in response to the label used by the reliable informant they looked towards the object that she had labelled and even in response to the label used by the unreliable informant, they looked towards the object that she had labelled. There were no differences in children's looking behavior in response to the reliable informant compared to the unreliable informant. This excludes the assumption that children would simply block 
information from an unreliable source, as children apparently attended to the information that was offered by the unreliable informant.

Similarly, the children were able to solve the Disambiguation eye-tracking task in Study $1 \mathrm{~b}$ based on testimony from either informant. In response to the novel label, they looked at the novel object when it was juxtaposed with the object that was labelled by reliable but also when juxtaposed with the object labelled by unreliable. Again, there were no differences between conditions: children solved the task based on testimony from the reliable source just as easily as based on testimony from the unreliable source. This adds further support to the conclusion, that children did not simply block the formation of any associations between the label used by the unreliable speaker and the object labelled by her.

Even in the explicit Recognition task, children's performance was significantly better than expected by chance with no differences between the two informants. Children were able to explicitly identify both the object that was labelled by reliable but also the object that was labelled by unreliable. It appears that when a reliable and unreliable informant offer information that is not perceived to be in conflict with each other, children will attend to and learn information from both in the same way.

This suggests that children's selective social learning is more nuanced than assumed by the Blocking and even the Selective gating account. It seems that in Study $1 \mathrm{~b}$, there were no general differences in encoding of testimony from reliable 
and unreliable informants. Rather, children learned from the previously inaccurate source in the same manner as from the previously accurate informant. There were no differences at all in the looking behavior across the whole time course in either of our eye-tracking task, and no differences in explicit reliance on testimony from the two sources in the interactive task. But does this mean that children only differentiate between the two sources if they perceive a contradiction in testimony from the two sources in the acquisition phase, and if they provide compatible information children will learn from both identically and discount the accuracy information? Against this interpretation already speaks the fact that almost all the children in Study $1 b$ were able to explicitly identify the more reliable informant in the manipulation check at the end of this study, suggesting they did retain accuracy information and just did not use it. Given the slight changes we had to make in this study in order to be able to investigate children's learning from unreliable sources who offer uncontested information, a direct comparison to our paradigm with conflicting information from the two sources in Study 1a is only meaningful to a limited extend. To further explore the role of a perceive contradiction in testimony, I used a between subject design were I provided children with either compatible or contradictory testimony from the two sources to test children's preferential reliance in a forced-choice task. 


\subsection{Study 1c}

The aim of Study 1c was to directly compare how children react to informants with differing history of accuracy when they offer conflicting or non-conflicting testimony during the acquisition phase. To investigate the role of contradictory testimony during the acquisition, half of the children in Study 1c were provided with contradictory testimony by the two sources, i.e. the same novel label but for two different objects just like in Study 1a. The other half of children saw the informants provide compatible information, that is two different labels for two different novel objects as in Study $1 \mathrm{~b}$. To test their selective reliance or disregard for information from either source, I conducted a Contrast task as in Study 1a. I presented children with both objects labelled by both informants and a male voice asked for the referent of a novel label. Based on findings in Study 1a, I expected children to selectively discount information offered by the unreliable informant in favor of the novel label provided by the naïve informant. If the conflict between information from differently reliable sources was the driver of children's selectivity, I assumed children to select the referent of the novel label at chance when both informants offered compatible information. This would suggest that children in fact did learn from the two sources identically in case they provided compatible information and that in this case children were biased to trust and discounted accuracy information. If however children still expected the novel label by the naïve informant to refer to the object previously labelled by the unreliable informant, this would suggest that children did retain accuracy information even 
if the unreliable source's testimony was not contradicted. Further, it would suggest that children can use this accuracy information between retrieval and reproduction of testimony to adjust their reliance at expression, even in a situation where in Study $1 \mathrm{~b}$ they appeared to learn from both sources in the same manner. Their reliance on the unreliable source in study $1 \mathrm{~b}$ would thus be due to rational inferences that led children to accept testimony from an unreliable source despite being aware that it might be inaccurate, but preferring to rely on potentially inaccurate testimony to not relying on testimony at all.

\subsubsection{Method}

\subsubsection{Participants}

Sixty-four monolingual German children $(\mathrm{M}$ age $=65.0$ months, range $=60.2-71.5$ months, 32 female were included in the final sample of this study. Participants were recruited from a database of children whose parents gave consent for their children to participate in child development studies at their kindergarten. None of the participants had known visual or auditory impairments. 


\subsubsection{Set up}

This study was conducted with preschoolers in their kindergartens. As the 5-yearolds in Study $1 \mathrm{a}$ and Study $1 \mathrm{~b}$ were able to answer the explicit questions in the interactive test phases with relative ease, I only used an interactive task in this study. This was done in form of a presentation of video clips like in the previous studies, followed by a touchscreen task where the children had to select one of the two objects on screen to answer the question of the experimenter. As presentation software I used Microsoft Powerpoint.

\subsubsection{Procedure}

At the beginning of this experiment, the child solved a brief task where the objects that would be labelled later on in the videos moved fast across the screen and the child was instructed to try to touch it with their finger. After this familiarization with the novel objects and the touchscreen procedure, children were introduced to both informants and their labelling accuracy in the History phase. Then both informant proceeded to provide novel testimony. For half of the children, this novel testimony was contradictory, i.e. the same novel label but for different objects. For the other half of children it was compatible, i.e. two different novel labels for two different objects. To investigate their selective preference for information from either source, I conducted two trials of the Contrast task from Study 1a. Before each test trial, the children solved two trials with two familiar 
objects. The attention getter video of balloons to piano music was also included in the study, after the warm up and before the test trials.

\section{History phase}

To familiarize the children with the accuracy of the two informants, they saw similar clips as in the previous studies. Children first saw one of the informants label three objects in turn, then the other informant labelled those same three objects in reversed order, followed by each labelling a fourth object. If the speaker was reliable, she used the correct label to refer to the objects visible on screen. If she was unreliable the label she used did not refer to the object present on screen, but was from the pool of familiar labels used by the reliable informant during the experiment. The identity of the reliable informant and order of the informants was counterbalanced across infants. Each of the film clips of the History phase lasted $10 \mathrm{~s}$.

\section{Acquisition phase}

The Acquisition phase in Study 1c was very similar to that of the previous studies. The main difference was that for half of the children the informants now provided compatible novel testimony, for the other half contradictory testimony. To teach the children the novel labels, they saw four videos (two with each informant) where the informants provided labels for the novel objects in the same manner as in Study 1a, with each video lasting 10.5s seconds. As labels, 2-syllabic pseudowords were used, Toma as the label used by both in the contradictory 
condition, and Schufi (Shoofi) and Mito as labels in the compatible condition. The novel objects in this study were from the NOUN Database (objects \#2044 and \#2048) (Horst \& Hout, 2016). Which label the reliable speaker used and which novel object she referred to was counterbalanced across children.

\section{Contrast task}

The test phase in Study 1c consisted of two trials of the Contrast task I used in Study 1a to investigate children's reliance on contradictory testimony from accurate and inaccurate sources (see Figure 11 III.). Both objects that were labelled by the two informants appeared on screen. The image of each object measured 350 x 350 pixels. The objects appeared on screen point-symmetrical to the center of the screen in the top left and bottom right corner. Location of the objects was counterbalanced across two trials. On each of the two trials, the male voice asked the children for the location of the referent of a label that the children had not heard before. Like in the previous studies, each of the two trials differed slightly in phrasing of the question asked by the male voice ("Where is the Blicket, the Blicket?" versus "Can you see the Blicket, the Blicket). A manipulation check identical to the previous studies was included. $96.9 \%$ of the 64 participants who gave an answer passed this manipulation check. 


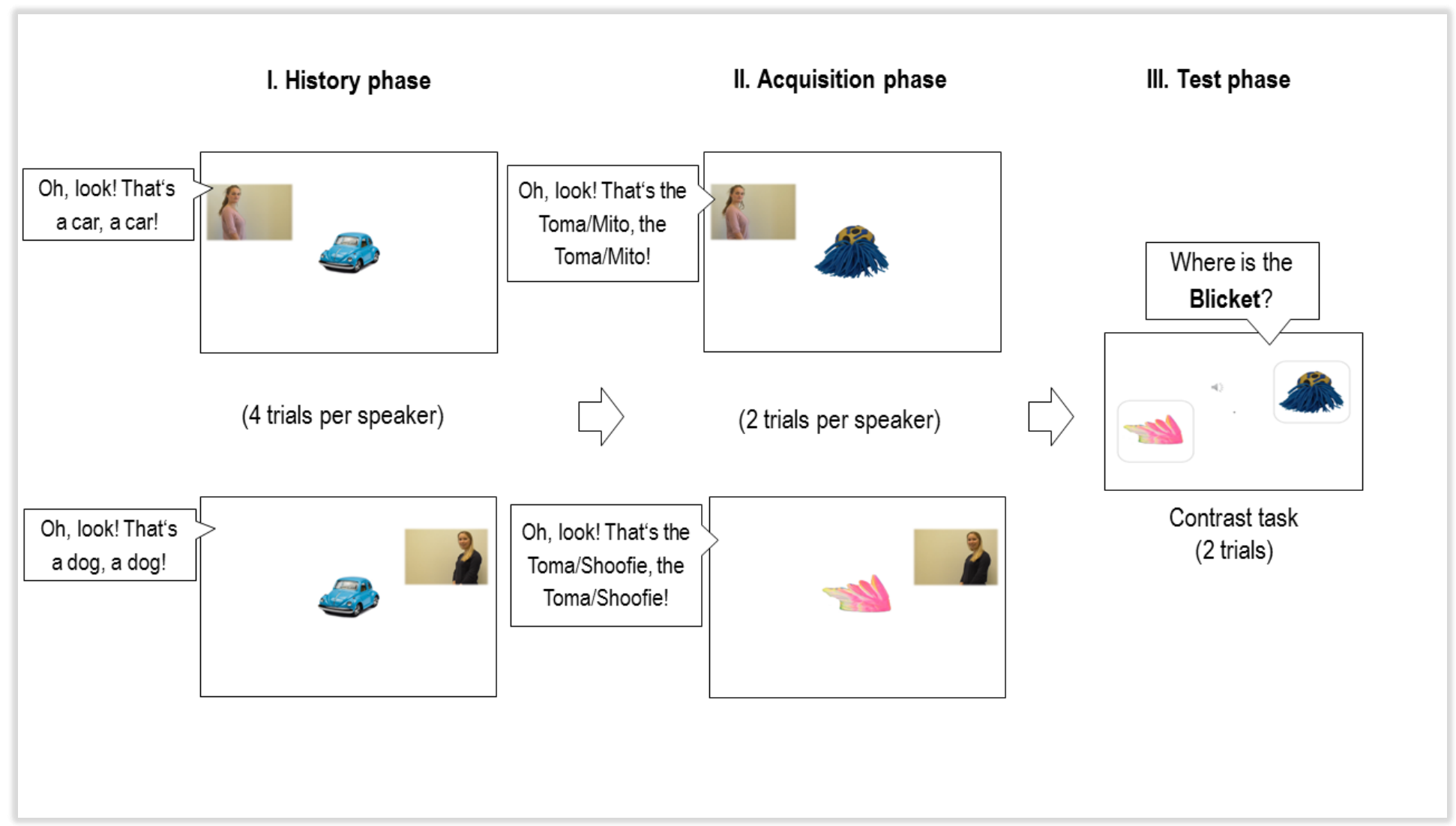

Figure 11. Schematic representation of the procedure in Study 1c; I) History phase; II) Acquisition phase; III) Test phase.

\subsubsection{Analysis and results}

I counted children's choices, which object they selected as the one referred to by the novel label used by the naïve informant. From their choices in two trials of this task I calculated a composite score where each choice of the object labelled by unreliable was counted as +1 , each choice of the object labelled by reliable was counted as -1 . The resulting composite score thus ranged from -2 (selecting the reliable object in both trials) to +2 (selecting the unreliable object in both trials) This composite score was used as dependent measure for the Disambiguation Task. 
Children's choices did not differ significantly depending on whether the two informants offered contradictory or compatible information (Mann-Whitney: $W=$ $551, p=.9$ ). Across the whole sample, children selected the object previously labelled by unreliable at rates above chance (One-sided Wilcoxon signed rank test: $V=387, p=.021)$ (see Figure 12).

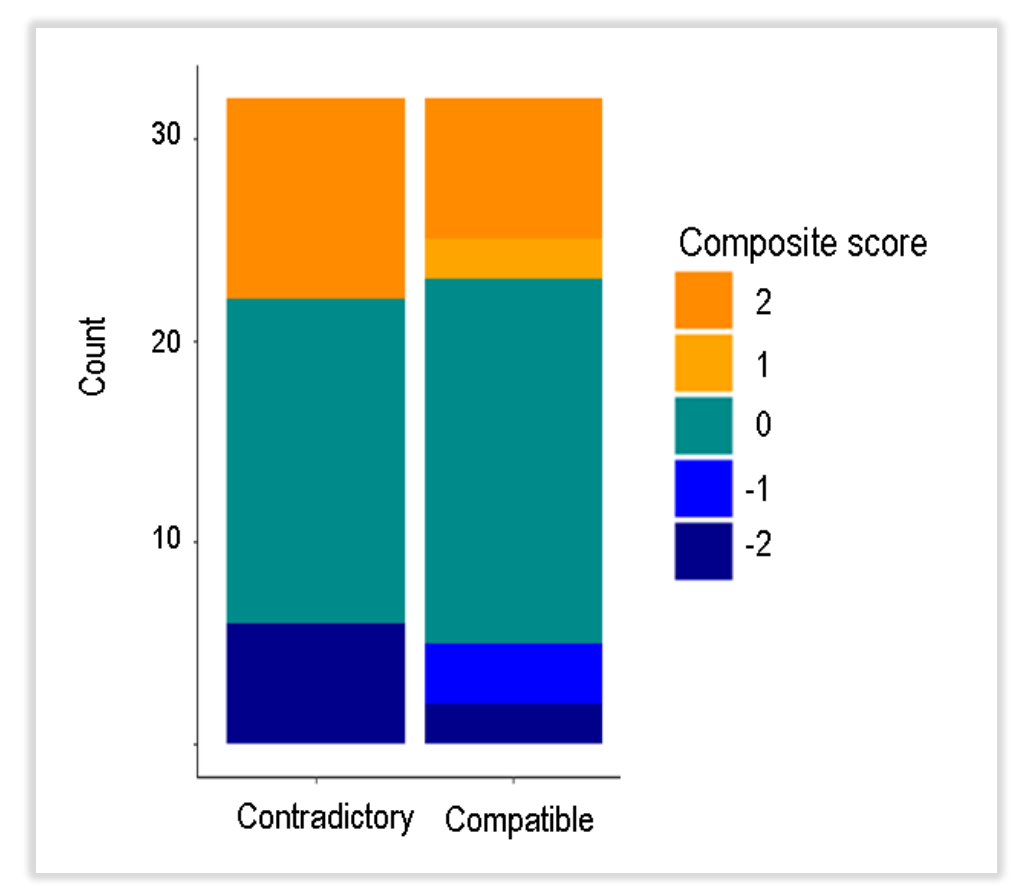

Figure 12. Composite score of two trials of children's object choice in the Contrast task by condition (Contradictory: reliable and unreliable informant used the same novel label to refer to two different novel objects; Compatible: reliable and unreliable informant used two different novel labels to refer to two different novel objects). 
This finding also holds if we only look at the first trial: there is again no difference between contradictory or compatible information (Mann-Whitney: $W=613, p=$ 0.467), and across the whole sample, children selected the object labelled by unreliable above chance (One-sided Wilcoxon signed rank test: $V=997.5, p=.031$ ) (see Figure 13).

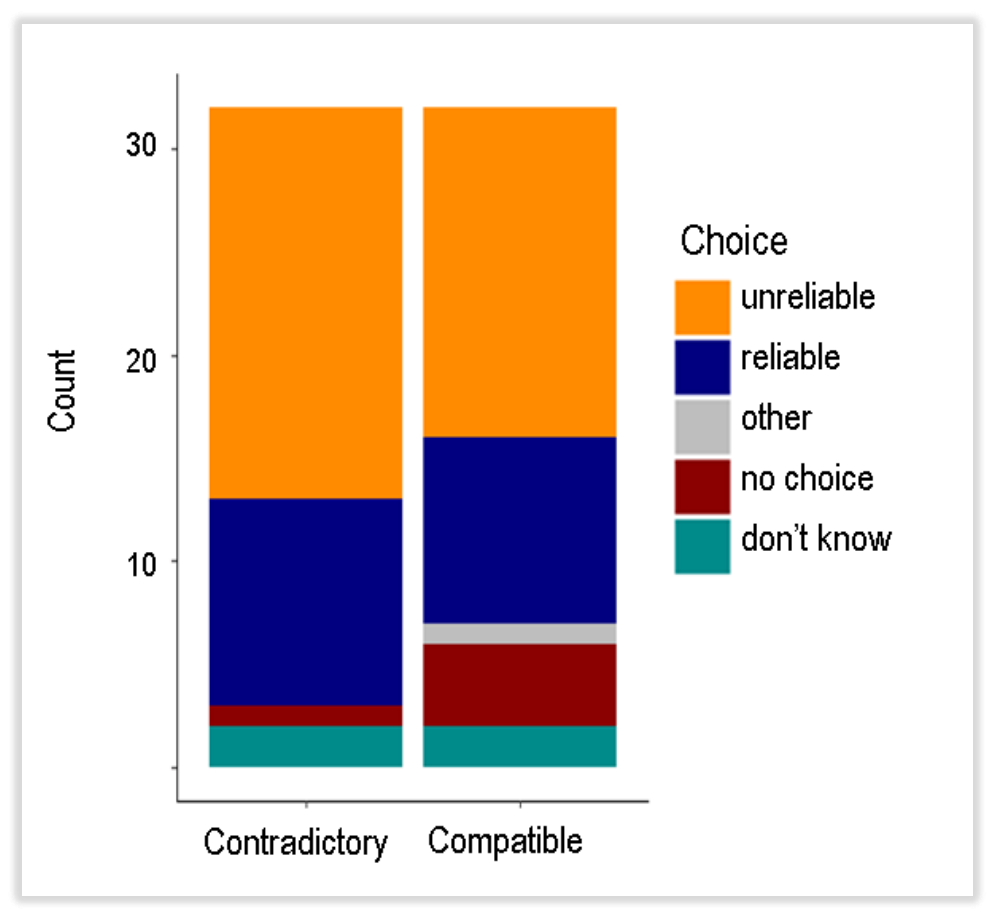

Figure 13. Children's object choice in the first trial of the Contrast task by condition (Contradictory: reliable and unreliable informant used the same novel label to refer to two different novel objects; Compatible: reliable and unreliable informant used two different novel labels to refer to two different novel objects). 


\subsubsection{Discussion}

Children in this study generally assumed the novel label provided by the male voice with unknown reliability in the Contrast task to refer to the object labelled by the previously inaccurate informant. It did not make a significant difference for children's performance, whether the accurate informant provide testimony that can be understood as a better alternative to testimony from the unreliable source or whether both provided compatible information. Already in their first trial of the Contrast task, children resolved the disambiguation by discounting testimony by the unreliable source in favor of novel testimony by an unknown source.

This shows that children's selectivity is not necessarily based on a contradiction in testimony by differently reliable sources when they acquire such information. Rather, even when an unreliable source provided uncontested testimony, which as we know from Study $1 \mathrm{~b}$ children generally attend to and rely on, children appear to retain (in-)accuracy information. In case novel testimony surfaces which now calls in question the validity of testimony by the unreliable source, children can reevaluate and adjust their reliance on testimony between retrieval and reproduction.

These findings suggest that preschoolers' selective trust in testimony is more nuanced than assume by both the Blocking or the Semantic gating account. Children can use accuracy information at expression when choosing whether or not to rely on testimony from an unreliable source, even if testimony from this source was uncontested during the acquisition. Typically, this leads to acceptance 
of such testimony to the same degree as from a reliable source, but in Study 1c, I could show that this does not entail that children are biased to rely on uncontested testimony from any source and discount accuracy information. Rather, children's acceptance of testimony from an unreliable source seems to be based on rational inferences leading children to the decision that uncontested testimony even if it might be inaccurate is still better than no information at all.

In the following Study Set 2, I was interested in how infants' achieve selectivity in their reliance on social information, specifically whether they rely on similar strategies as preschoolers. 


\section{Study Set 2: Early Development of Selective Trust in Testimony}

\subsection{Introduction}

Preschoolers attribute (un-)reliability to particular individuals, keep this information in mind and use it to endorse testimony from particular sources over testimony from others. They show this behavior both when prompted to consider source information and when spontaneously reacting to testimony from different sources. Paradigms that have been typically used with infants cannot conclusively tell whether infants rely on the same strategies or whether they simply learn less in confusing situations. These paradigms usually confront infants with a single reliable or unreliable informant, possibly to reduce the task demand. In my Study Set 2, I aimed to investigate whether infants like preschoolers are able to ascribe (un-)reliability to particular individuals. In order to be able to confront infants with two different sources that differ in accuracy while still keeping the task demands low, I introduced eye-tracking, specifically an intermodal preferential looking paradigm to research on selective trust in testimony in infants.

In Study 2a, I replicated typical paradigms that been used to investigate selective trust in testimony in older children. 2-year-old infants first saw two speakers label familiar objects, one of them accurately, the other one inaccurately. Then both speakers provided novel testimony in contradiction to each other, that is the same novel label but referring to two different objects. In the same two tasks that I used 
in Study 1a, I explored infants' selective reliance on the testimony from the two sources. If infants used the same strategies as preschoolers, I expected them to selectively prefer the testimony from the reliable source. However, assuming that infants' selectivity in single informant paradigms could be due to confusion or situational variables, a performance at chance in Study 2a might not necessarily reflect a lack of attribution of reliability to particular informants. Rather, to investigate whether infants' selectivity in other studies was caused by confusion due to unusual assertions, I investigated infants' reliance on uncontested testimony by the same two sources in Study $2 \mathrm{~b}$. If infants are confused when confronted with inaccurate assertions, infants should fail to learn from any source in Study 2b. However, if children do not ascribe reliability to individual sources, we might see infants failing to be selective in Study 2a while learning from both in Study $2 b$ when they offered testimony that is not in contradiction with each other.

\subsection{Study $2 a$}

The aim of Study 2a was to investigate whether infants like preschoolers attribute reliability to particular individuals. Therefore, I conducted a paradigm with two informants that differ in their history of labelling accuracy. In studies like that, preschoolers typically show a preference from information from the more accurate source. Studies with infants show that they also learn less when confronted with an unreliable informant. However, these studies cannot be used to conclusively determine whether infants rely on similar strategies as preschoolers. Presumably 
to keep the task demands low, research with infants has focused on between subjects design. This however allows for alternative explanations of infants' selectivity. In order to keep the task demands low, I used eye-tracking to investigate infants' attribution of reliability to particular individuals.

In Study 2a, I presented children with a video presentation of two female informants labelling familiar objects accurately or inaccurately. Then both informants provided contradictory testimony, i.e. the same novel label, but for two different objects. To find out about whether and how children rely on this information, children were tested in four tasks, two eye-tracking tasks followed by interactive versions of these tasks. This study was very similar to Study 1a I conducted with 5-year-old infants, with the only difference being a prolonged label training.

Infants solved eye-tracking and interactive versions of both the Endorsement task and the Contrast task. In the Endorsement tasks, they were asked for the referent of the label both informants had used between the two objects both had referred to with that label. If infants preferentially endorsed information from a previously accurate source, I would expect them in the eye-tracking task to look towards the object that had been labelled by the reliable informant upon hearing the label used by both. Similarly, in the interactive task, infants should select the object labelled by the reliable informant as the referent of the label used by both. In the Contrast tasks, the male voice asked for the referent of a different, completely novel label and infants were presented with both objects that the informant had labelled. If 
they relied on similar strategies as preschoolers, I would expect infants to assume that the novel label to referred to the object labelled by the unreliable speaker, rather than the object labelled by the reliable one, both in the eye-tracking and the interactive version of this task.

\subsubsection{Method}

\subsubsection{Participants}

Thirty-six monolingual German infants $(\mathrm{M}$ age $=24.0$ months, range $=23.1-25.9$ months, 17 female) were included in the final sample of this experiment. Participants were recruited from a database of infants whose parents gave consent to be invited for scientific studies by our department. None of the infants had known visual or auditory impairments. Parents gave informed consent for participation of their infant in the study. Three additional infants were tested but could not be included in the final sample, two as they stopped participating before the conclusion of the experiment and one as the eye-tracker did not record gaze data due to technical issues.

\subsubsection{Set up}

The setup in this study was highly similar to the setup in Study 1a. For the eyetracking tasks, I used a SMI Red250 eye-tracker attached to a 24-inch TFT flatscreen monitor with a display resolution of $1920 \times 1080$ pixels. During the 
experiment, participants sat on car seat for infants mounted on an adjustable chair to ensure that the infant's eyes were inside the detectable range of the eye-tracker. The calibration and validation procedure was identical to previous studies. EPrime 2.0 was used as presentation software. The novel objects that infants handled, and saw still pictures of, were plush toys representing microbes (a green plush toy, representing Euglena, and a blue plush toy, representing Giardia).

\subsubsection{Procedure}

The session started with a warm-up phase, in which the infant played with the two experimenters (E1 and. E2). During this phase, the infant was allowed to play for up to 30 seconds with either plush toy that would later be named by the reliable and unreliable informants. Subsequently, each test session consisted of the following phases. First, infants were introduced to both informants and their labelling accuracy in the History phase. In the Acquisition phase, both informants proceeded to provide novel testimony, that is novel labels for novel objects. In my first study with infants, the information provided by the two informants was contradictory, i.e. they offered the same novel label but for different novel objects. Infants' appreciation of testimony from the two sources was then investigated in two eye-tracking tasks: the Endorsement and Contrast task. Finally, infants participated in interactive versions of these tasks. In the very beginning of the experiment and as a transition scene after the History phase and after the 
Acquisition phase, the same attention getter video of balloons to piano music as in the previous studies was included in this study.

\section{History phase}

At the beginning of the video presentation, the infants saw a 10s greeting part to introduce the two informants as in previous studies. To familiarize the children with the accuracy of the two informants, they saw 8 video clips (4 with each informant) where the actresses labelled familiar objects (apple, baby, car, dog), just like in Study 1a and 1b. Infants first saw one of the informants label three objects in turn, then the other informant labelled those same three objects in reversed order, followed by each labelling a fourth object. If the speaker was reliable, she used the correct label to refer to the objects visible on screen. If she was unreliable the label she used did not refer to the object present on screen, but was from the pool of familiar labels used by the reliable informant during the experiment. The identity of the reliable informant and order of the informants was counterbalanced across infants. Each of the film clips of the History phase lasted 10s.

\section{Acquisition phase}

The label training in Study 2a was very similar to Study 1a and $1 \mathrm{~b}$. The main difference was that I increased the number of times the informants labelled the object and thus the duration of this phase. In the Acquisition phase, the informants proceeded to teach the infant the same novel label, but to refer to different novel 
objects. As novel label, “Toma” was used here in Study 2a. The novel objects were the same we used in Study 1a (Euglena (green) and Giardia (blue)). Each infant saw two videos (one with each informant) with each video lasting 32.5 seconds. At the start of each clip, an unfamiliar object appeared in the bottom corner of the screen. Then one of the informants appeared on the screen on the opposite side of the object and addressed the infant (saying "Oh, guck mal!” (“Oh, look!")). Then she turned to point at the object, labelling the novel object "This is the Toma, the Toma!" ("Das ist ja das Toma, das Toma!"). Then the object moved towards the other corner of the screen and finally to the center of the screen where the object was labelled in the same manner a second and a third time ("Oh, look! Now the Toma is here, the Toma!" - "Schau mal! Jetzt ist das Toma hier, das Toma!") (“Oh, look! And now the Toma is here, the Toma!" - "Oh guck mal! Und jetzt ist das Toma hier, das Toma!"). Which novel object the reliable and unreliable speaker referred to was counterbalanced across infants.

\section{Reminder}

After the Acquisition phase, two trials of History phase with additional familiar objects (baby, car) and the whole Acquisition phase was repeated, to serve as reminder. 


\section{Eye-tracking test phase}

The infants then participated in two eye-tracking tasks, first the Endorsement task and then the Contrast task (consisting of 2 trials per task). In both tasks the two novel objects, that the speakers had labelled previously, appeared on screen and infants heard a male voice asking for one of them (see Figure 14 III). In the Endorsement task, the male speaker asked for the "Toma" (i.e., the novel label that had been used by both informants), whereas in the Contrast task, he used another completely novel two-syllable word, and asked for the "Blicket". I conducted two trials of each task with each infant. Before each trial of the Endorsement task and the Contrast task, a fixation-cross appeared to guide the infant's gaze to the center of the screen. The objects were presented on the left and right side of the screen, symmetrical to the center of the screen. The image of each object measured $344 \mathrm{x}$ 696 pixels. The location of the objects was counterbalanced across the two trials of each task. The recording of the male voice was timed such that the objects were visible on screen for a preview time of $2.5 \mathrm{~s}$ before label onset. Total duration of these videos was 5.5s. Trials with two familiar objects and the male voice asking for one of the two objects were interspersed before each test trial to keep the compliance high. 


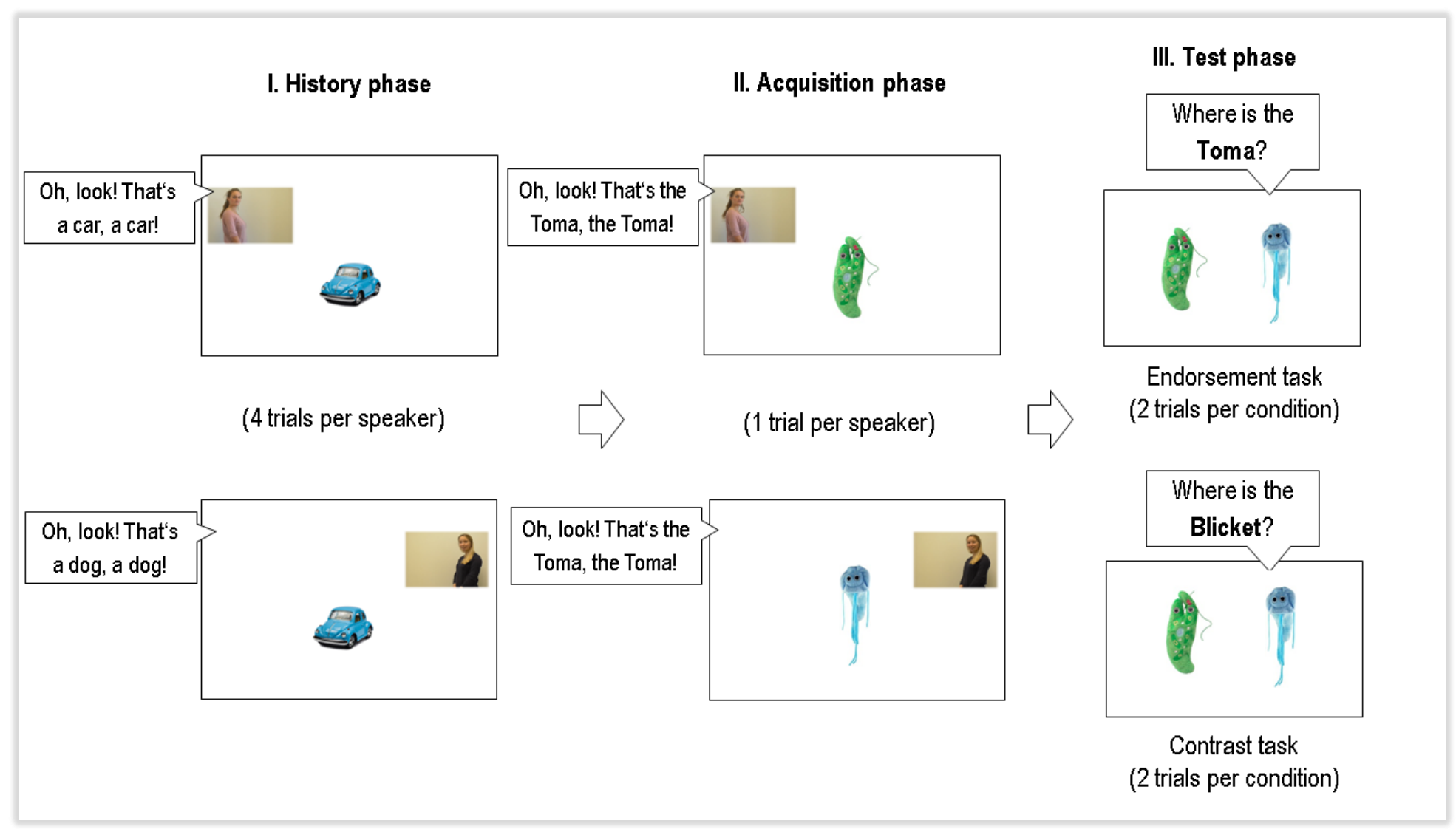

Figure 14. Schematic representation of the procedure in Study 2a; I) History phase; II) Acquisition phase; III) Test phase.

\section{Interactive test phase}

After completion of the eye-tracking part, the experimenter and the infant moved to a table in an adjacent room where the Interactive task was conducted. In this task, the infants were instructed to select one of two objects from a tray and put in on a chute behind them. After one trial of demonstration of how the chute works with a familiar object, two more trials with familiar objects were conducted with the infant. On each of those trials, the infant had to select a familiar target object among a familiar distractor. Following the familiarization with the procedure, two test trials were conducted where the plush toys, of which images were presented 
during the eye-tracking tasks, were used. Both objects were presented to the infants on a tray in counterbalanced order. In the first test trial, E1 asked the infant to put the "Toma" on the chute, in the second the "Blicket". Between the two test trials, another trial with a familiar object was interspersed.

\subsubsection{Analysis}

\section{Eye-tracking tasks}

I conducted both a time window analysis where we compared children's target fixations before and after label onset and a bootstrapped cluster-based permutation analysis of the whole time course. I defined areas of interest (AOI) for both locations left and right the center of the screen, where the images appeared during this task, as the size of the image plus $2.5 \%$ of the screen dimensions as overlap towards each side. Given an image size of $344 \times 696$ pixels and screen dimensions of $1920 \times 1080$ pixels, the dimensions of each AOI were defined as 440 x 750 pixels. There was no spatial overlap between the two AOIs. With a sampling rate of $60 \mathrm{~Hz}$, the eye-tracker provided the $\mathrm{x}$ - and y-coordinates of participants' gaze direction for every $16 \mathrm{~ms}$ for both eyes individually. These coordinates were averaged across both eyes, unless the coordinates for one eye were missing (e.g., when due to participants' movements only one eye was detected by the eyetracker). These gaze coordinates were then compared to the two AOIs, such that each data point was coded for whether the infants looked at the target image or the distractor image, or anywhere else. In order to remove trials where infants did 
not pay attention to the video presentation, I excluded trials in which infants did not look at the screen for at least half of the trial's duration. This led to the exclusion of three trials of the familiar object trials, one trial of the Endorsement task and four trials of the Contrast task.

Gaze data was aggregated across two time windows. The pre-naming window was a baseline window that counted all fixations $2000 \mathrm{~ms}$ before label onset $(500 \mathrm{~ms}-$ $2500 \mathrm{~ms}$ after video onset). The second window, the target window, counted all fixations from $240 \mathrm{~ms}$ after label onset (to adjust for reaction time and processing) to $2240 \mathrm{~ms}$ after label onset (2740ms $-4740 \mathrm{~ms}$ after video onset). For both windows, we determined the time that infants spent looking at the target or at the distractor. To calculate the proportion of target looking, the time infants spent looking at the target was divided by the total looking time at both target and distractor and averaged across the two trials of each task. The target in the familiar trials was, of course, the object to which the uttered label referred, and in both the Endorsement and the Contrast task, the object labelled by reliable was denoted as target. Hence, the object labelled by the unreliable speaker was denoted as distractor object in the Endorsement and Contrast task.

As this analysis relies on artificial time windows, I also computed a bootstrapped cluster-based permutation analysis (von Holzen \& Mani, 2012) to model infants' gaze behavior across the time course of each trial more directly as in the previous studies. To calculate the time course analysis, I used the eyetrackingR package (Dink \& Ferguson, 2018). The resampling rate was 1000 samples. 


\section{Interactive tasks}

As dependent measure for the Interactive Tasks, I counted infants' choices, which object they selected as the one referred to by the label used by both informants and which one they selected as the one referred to by the novel label. I calculated a McNemar's test to compare infants' object choice between the two tasks, and binomial test to compare it to chance.

\subsubsection{Results}

\section{Eye-tracking tasks}

I predicted that, if infants considered the speakers' previous accuracy, they should show the following response pattern: In the Endorsement task, when they heard the trained novel label "Toma", they should look at the target object, i.e., the one the reliable speaker had labelled. In the Contrast task, when they heard the completely novel label "Blicket", they should look at the object labelled by the unreliable speaker, i.e. the distractor.

To test this, I first looked at infants' preferential looking time towards the target during the baseline window compared during the target window after label onset across both tasks and the familiar object trials. I calculated a 3×2 ANOVA with proportion of target looking as dependent variable, and time window (pre-naming vs. post-naming) and type of task (Familiar, Endorsement, or Contrast) as withinsubject factors. I found a main effect of type of task $\left(F(2,70)=13.2, p<.001, \eta_{p^{2}}=.27\right)$, 
a main effect of time window $\left(F(1,35)=16.85, p<.001, \eta_{p}{ }^{2}=.33\right)$, and a significant interaction effect of time window and type of task $\left(F(2,70)=5.56, p=.006, \eta_{p^{2}}=.14\right)$ (see Figure 15).

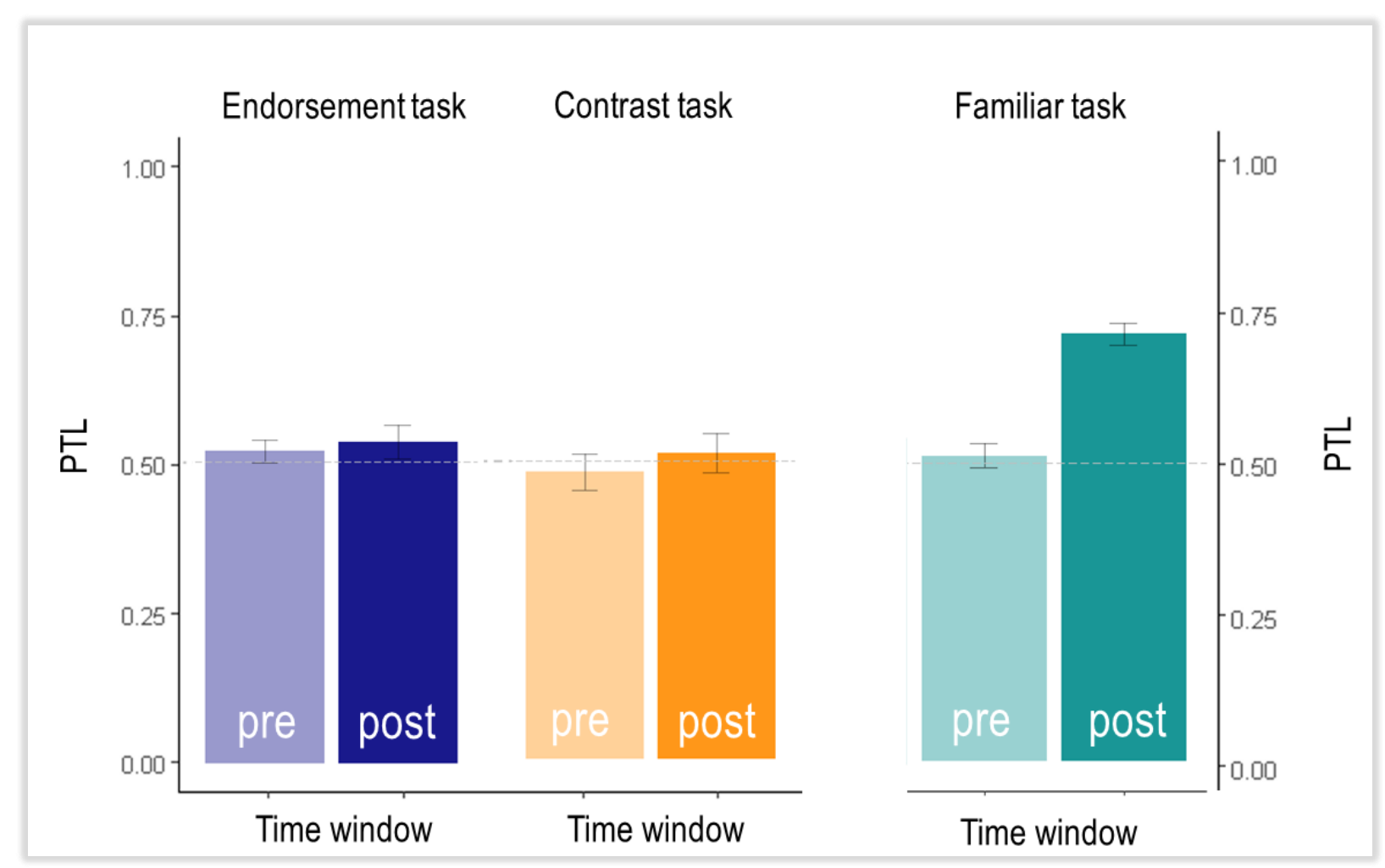

Figure 15. Proportion of target looking (PTL) in the Endorsement and Contrast task/ in response to the familiar label (target $=$ object labelled by reliable/familiar object). PTL calculated as time spent looking at the target object divided by the time spent looking at target plus at distractor. Time windows in relation to label onset (pre- label onset/ postlabel onset). Horizontal dotted line represents chance level. 
As the infants had no problems identifying the familiar objects, I then removed this task from the analysis and compared infants' performance in the Endorsement and Contrast tasks directly. I calculated a 2x2 ANOVA with proportion of target looking as dependent variable, and time window (pre-naming vs. post-naming) and type of task (Endorsement vs. Contrast) as within-subject factors. There were no significant effects of type of task $(F(1,35)=0.97, p=.33)$ or time window $(F(1,35)=$ $0.84, p=.37)$, and no interaction between the two factors $(F(1,35)=0.06, p=.82)$.

The time course analysis presented the same picture. There were no significant differences in infants' looking behavior between the Endorsement and the Contrast tasks (see Figure 16). 


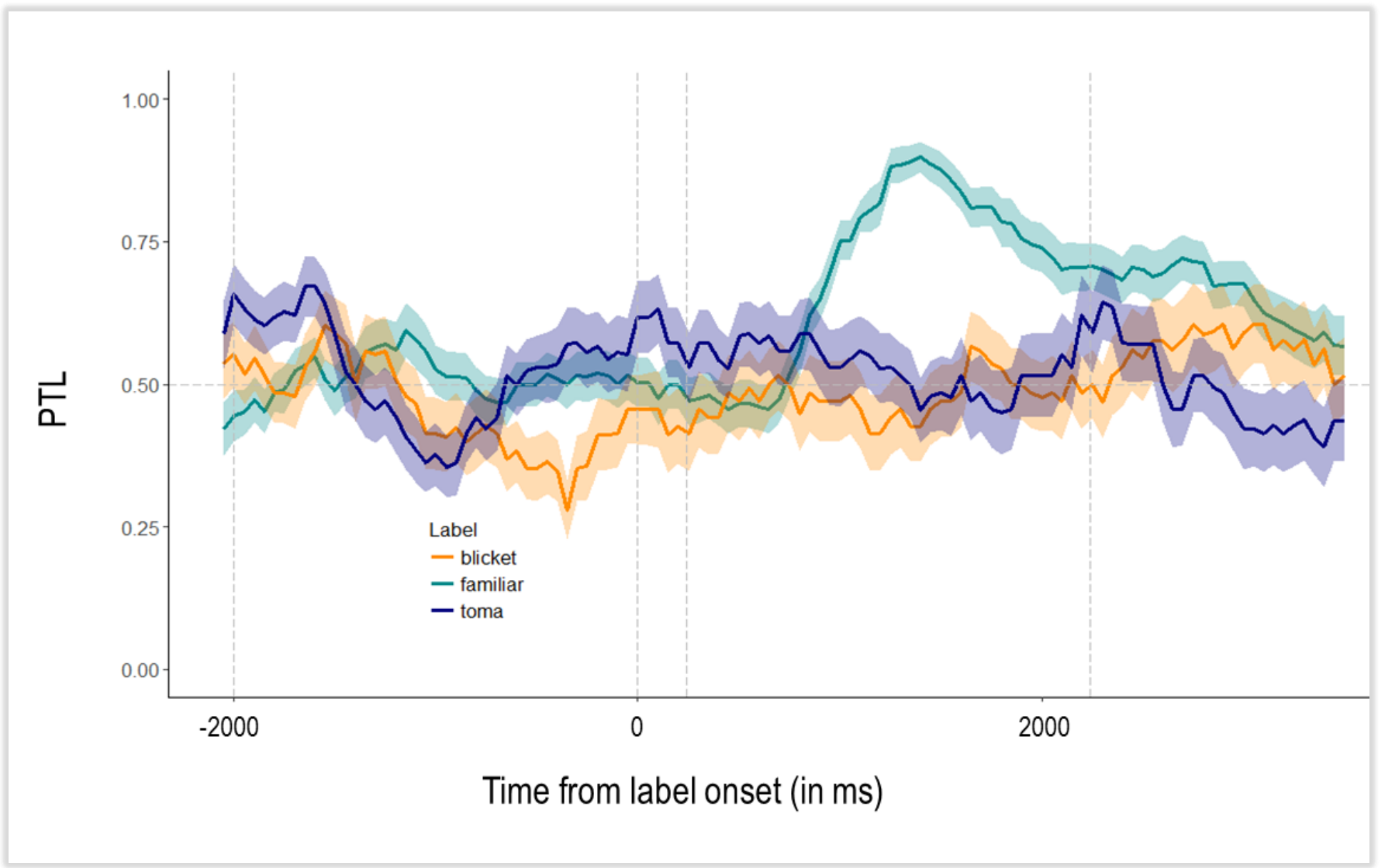

Figure 16. Proportion of target looking (PTL) across the time course in the eye-tracking Endorsement (blue line) and Contrast (orange line) tasks and the familiar label trials (green line). Vertical dotted lines represent time windows used for time window analysis (pre-naming window: -2000 to $0 \mathrm{~ms}$ from label onset; post-naming window: 240 to $2240 \mathrm{~ms})$, horizontal dotted line represents chance level.

\section{Interactive tasks}

The results of the interactive task support the finding that infants guessed which object was referred to by the label used by both and by the novel label. There were 
no significant differences between infants' performance in the two tasks (see Figure 16). Binomial tests showed that their response was not different from chance. Infants selected the object labelled by reliable as referent of the label used by both in 20 out of 37 cases, the object labelled by unreliable in 15 cases. As referent of the novel label, they selected the object labelled by reliable in 17 out of 37 cases and the object labelled by unreliable in 17 cases.

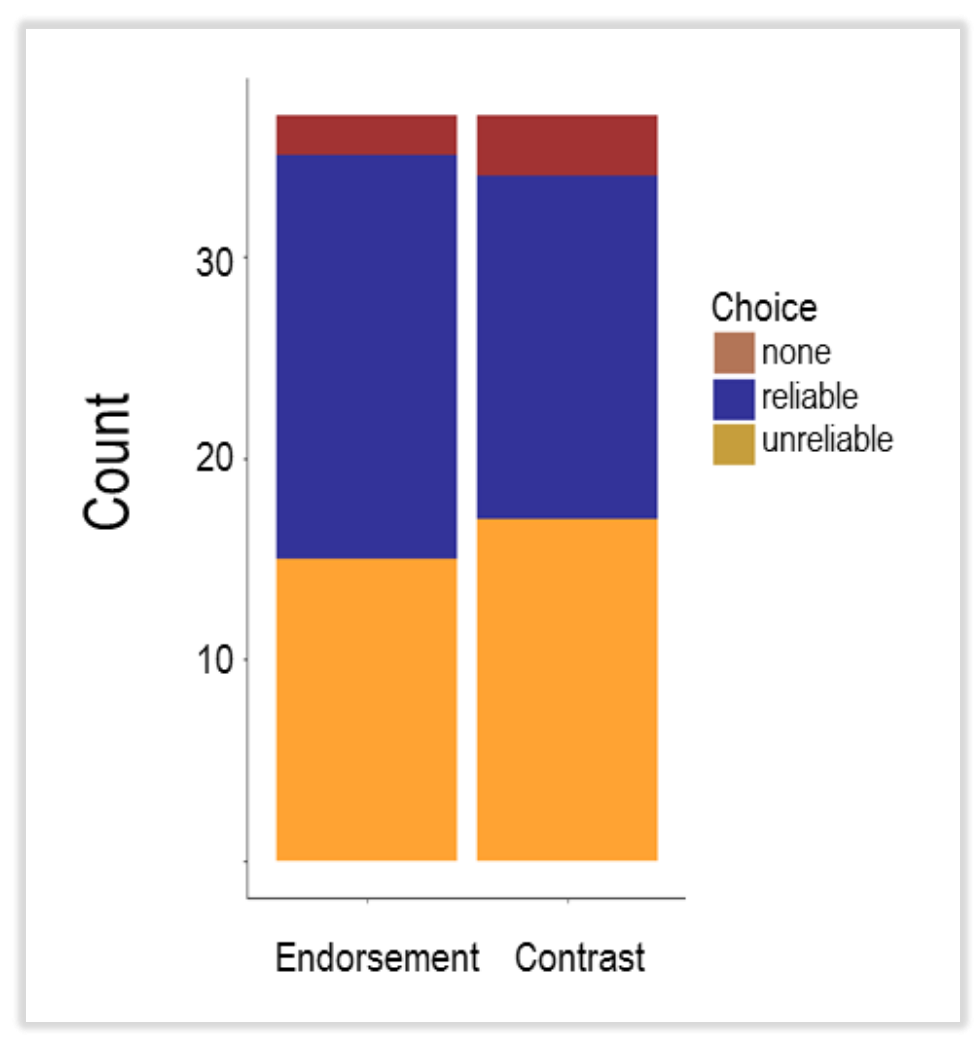

Figure 16. Infants' object choices in the interactive Endorsement and Contrast tasks. 


\subsubsection{Discussion}

Infants in Study 2a were not selective in their reliance on sources with distinct history of accuracy. In both the eye-tracking and the interactive Endorsement task, children did not selectively look towards or select the object that had been labelled by the reliable informants. Rather their performance was at chance. Similarly, in both the eye-tracking and the interactive Contrast task, infants did not expect the novel label to refer to any of the two objects. Both their looking behavior in the exetracking task and their choices in the interactive task did not differ from chance. Does that mean that the infants in my study did not understand that both informants differed in the accuracy of their testimony? Typically, at 24 months infants are able to detect mismatches of testimony and their own knowledge. Similar as in other studies, a few infants in our study reacted with social referencing or even protest to the unreliable informant labelling known objects incorrectly (Koenig \& Harris, 2007; Pea, 1982). It is thus not likely that a failure of error detection led to infants' performance at chance in this study.

It is however possible that the cognitive load in this study was too high for infants at 24 months leading to a failure in retaining any information. After all, infants would need to retain accuracy information about two sources additionally to information that was provided by the testimony of the two distinct sources. This might be too much for infants to keep in mind, especially as both sources provided completely novel and arbitrary information in form of pseudowords that the infants had never heard before as labels for objects the infants had never seen 
before. Alternatively, as infants are used to ostensive cues as markers of generalizable information, inaccurate testimony presented with such cues might confuse them. This confusion could in turn lead to reduced learning without an appreciation of any particular individual.

In order to exclude these alternative explanations and to investigate whether infants indeed do not attribute reliability to particular informants, I conducted a second study with the only difference to Study 2a being that the two sources now provided compatible testimony during the Acquisition phase. If infants' lack of differentiation between the two sources in Study 2a was due to general confusion or high task demands, I would expect a similar performance at chance when they learned information from the two sources that was not contradictory. If infants performance in Study 2a was based on a lack of reliability ascription, I would expect them to learn from both informants equally in Study $2 b$. This would be a hint that infants relied on different strategies than preschoolers when confronted with testimony by a previously inaccurate source.

\subsection{Study $2 b$}

In Study 2b, I aimed to exclude alternative explanations for infants' performance in Study 2a. To find out whether infants are confused by the presence of an inaccurate informant or whether the task demands in Study 2a were too high, I investigated whether infants encoded information from a previously accurate and 
a previously inaccurate source differently when they provided compatible information.

The accuracy of the two female informants in Study $2 b$ was introduced in the same way as in Study 2a. Then both informants provided information that was not in contradiction with each other, that is two novel labels but for two different novel objects. To find out about whether and how infants encoded this information, they were tested in two tasks, an eye-tracking tasks followed by an interactive version of this task.

In the eye-tracking Recognition task, infants saw the two objects on screen that had been labelled by the two informants and heard a male voice ask for the label that had been used by the reliable and the label that had been used by the unreliable informant subsequently. If infants encoded this information, I expected them upon hearing the label used by the reliable or the unreliable informant to look towards the object that had been labelled by the respective speaker. In the interactive Recognition task, the infants had to pick out of the two physical representations of the objects that had been labelled by the two speakers according to the labels that either had used.

If infants encoded information from the two sources differently, I would expect them to rely less on the object-label-mapping provided by the unreliable source. This assumption is based on the finding by Koenig and Woodward (2010) who could show that infants learned from both a reliable and an unreliable source at rates above chance, but from the reliable source significantly more than from the 
unreliable source. This would also suggest that infants rely on different strategies as preschoolers, who learned compatible information from both sources to a similar degree in Study 1b. If infants performance in Study 2a was based on high task demands or confusion, I would expect them to not learn from either source. If the performance in Study 2a however was due to them not attributing reliability to particular individuals I would expect them to learn from both in Study $2 b$.

\subsubsection{Method}

\subsubsection{Participants}

Thirty-five monolingual German infants $(\mathrm{M}$ age $=23.9$ months, range $=23.3-24.9$ months, 16 female) were included in the final sample of this experiment. Five additional infants were tested but could not be included in the final sample as the eye-tracker did not provide data for four of them due to technical issues and one stopped participating before the conclusion of the experiment

\subsubsection{Procedure}

The experimental setup was identical to Study 2a. The familiarization with the speakers' accuracy in the History phase and the novel label training in the Acquisition phase was also kept nearly identical with the only exception being that in the Acquisition phase, the two informants provided compatible information, 
that is two different novel labels (Toma, Shoofie) for the two different novel objects. Which label the unreliable speaker used and which object she referred to was counterbalanced across infants.

\section{Eye-tracking test phase}

In the eye-tracking tasks, the two novel objects that had previously been labelled by either speaker were visible on screen. The male voice now asked for the referents of the labels that either speaker had used, the "Toma" or the "Shoofie" (see Figure 17 III.). I conducted two trials of this Recognition task for both the label used by the reliable speaker and the label used by the unreliable speaker with each infant. 
I. History phase

II. Acquisition phase

III. Test phase

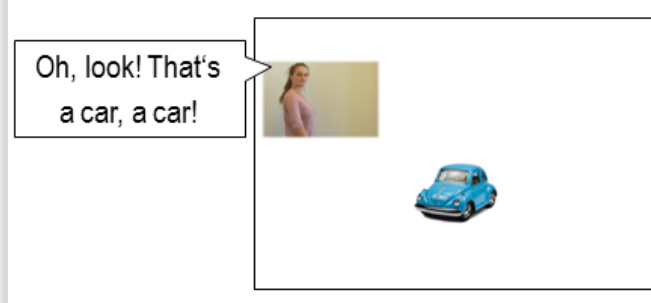

(4 trials per speaker)

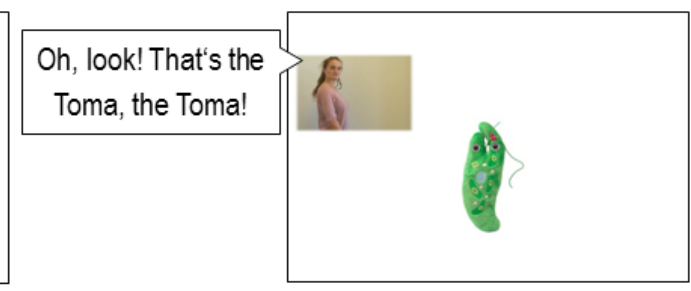

(1 trial per speaker)
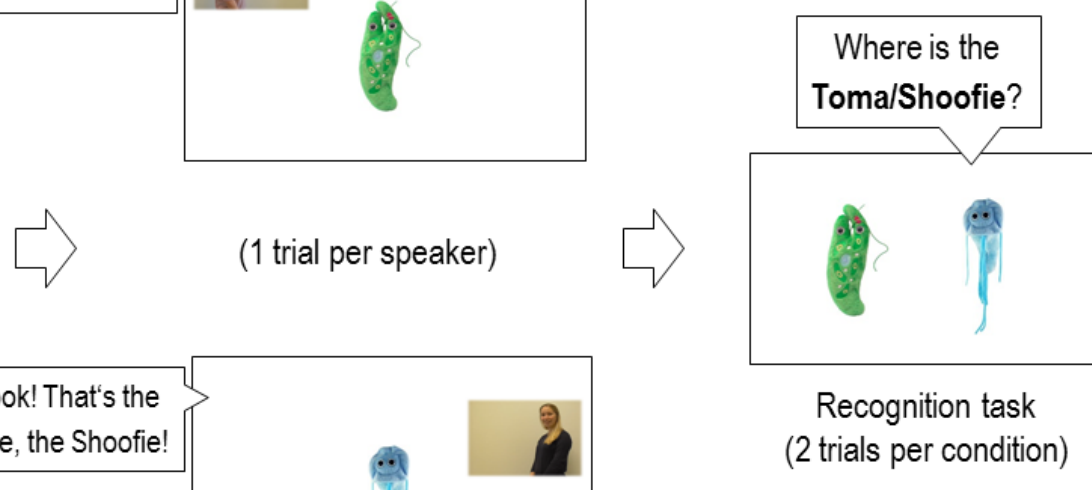

Figure 17. Schematic representation of the procedure in Study 2b; I) History phase; II) Acquisition phase; III) Test phase.

\section{Interactive task}

In the interactive version of our eye-tracking task, the experimenter asked the infants to select the referent of either novel label from the two novel objects. 


\subsubsection{Analysis}

\section{Eye-tracking test phase}

I again conducted both a time window analysis and a bootstrapped cluster-based permutation analysis of the whole time course. First, I compared infants' preferential looking time towards the objects labelled by reliable or unreliable 2 seconds before and 2 seconds after they heard they male voice utter the label in each task. With the time course analysis, I compared the distribution of clusters of significant differences across the whole duration of each trial to a random distribution of the empirical differences. Unfortunately, 31 trials of the familiar object trials, 18 trial with the target labelled by the unreliable source and 14 trials with the target labelled by the reliable source had to be excluded from the analysis. This was in part caused by the fuzziness of three infants in this study, but also due to unfavorable lighting conditions in the lab at the time of the conduction of this study.

\section{Interactive task}

As dependent measure for the interactive task, I counted infants' choices, which object they selected as the one referred to by the label used by either. I calculated a McNemar's test to compare the performance between the two labels, and binomials test to compare it to chance. 


\subsubsection{Results}

\section{Eye-tracking test phase}

To test whether infants were able to identify the objects that had been labelled by the reliable and unreliable speaker upon hearing the label used by either, I first looked at infants' preferential looking time towards the target during the baseline window compared during the target window after label onset across both tasks and the familiar object trials.

I calculated a 3×2 ANOVA with proportion of target looking as dependent variable, and time window (pre-naming vs. post-naming) and target (familiar object, object labelled by reliable, object labelled by unreliable) as within-subject factors. Six infants had to be removed from this analysis, as they did not provide data for every condition. We found a main effect of type of task $\left(F(2,54)=3.90, p=.026, \eta_{p}{ }^{2}=.13\right)$, a main effect of time window $\left(F(1,27)=14.26, p<.001, \eta_{p^{2}}=.35\right)$, and no interaction $(F(2,54)=0.18, p=.84)$ (see Figure 18). 


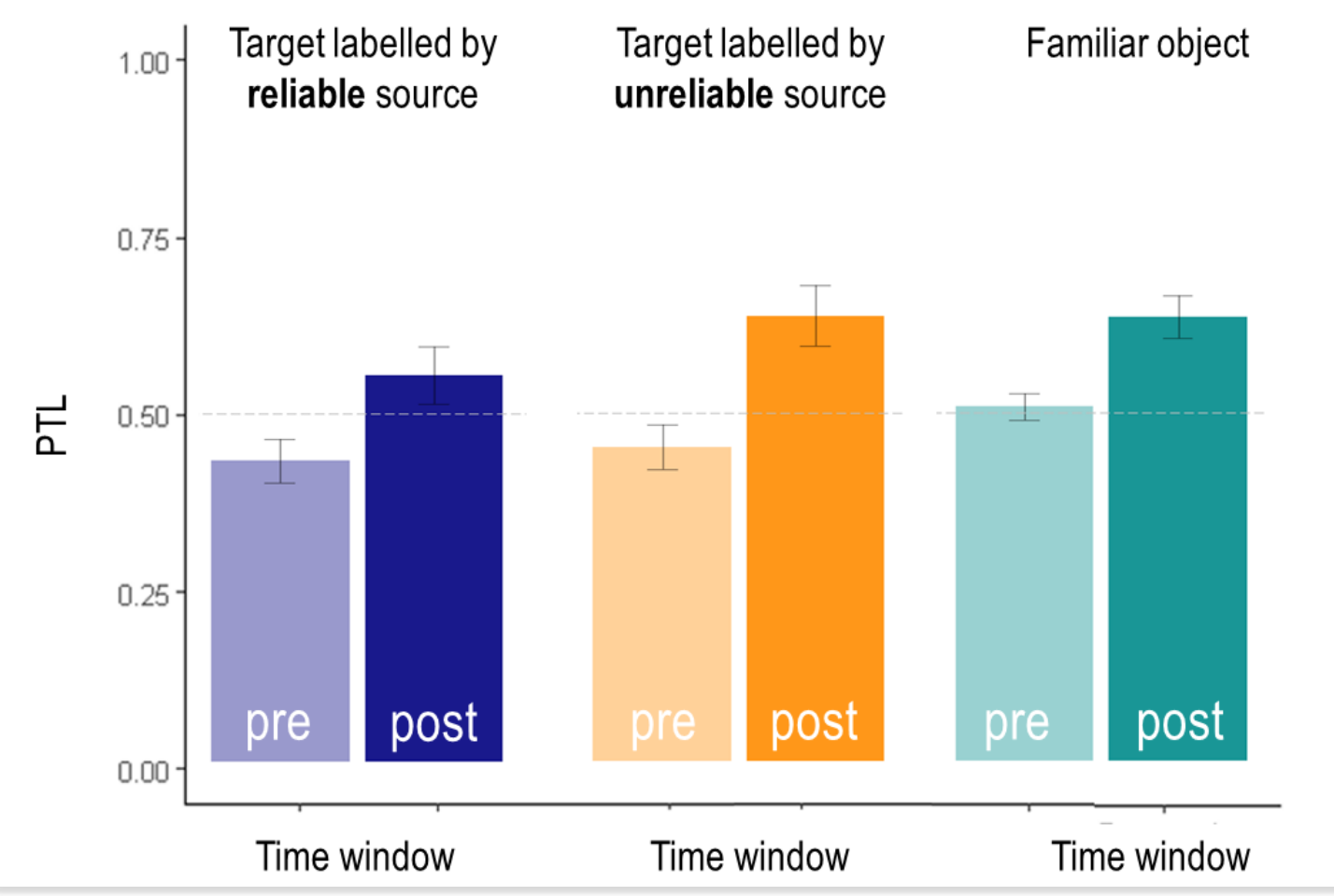

Figure 18. Proportion of target looking (PTL) in the Recognition task by condition (target object labelled by reliable, target object labelled by unreliable, target object familiar,). PTL calculated as time spent looking at the target object divided by the time spent looking at target plus at any of the distractors. Horizontal dotted line represents chance level.

Analogous to the analysis of the Recognition task in Study $1 b$, I then removed the familiar condition, to compare infants learning from the reliable and the unreliable informant directly. I calculated a 2x2 ANOVA with proportion of target looking as dependent variable, and time window (pre-naming vs. post-naming) and target (object labelled by reliable, object labelled by unreliable) as within-subject factors. Three infants had to be removed from this analysis, as they did not provide data for every condition. I found a significant main effect of time window $(F(1,28)=9.85$, 
$\left.p=.004, \eta_{p}{ }^{2}=.26\right)$. There was no main effect of target $(F(1,28)=1.54, \mathrm{p}=.23)$ and no significant interaction of time window and target $(F(1,28)=0.25, p=.62)$.

The time course analysis showed that infants' looking behavior did not differ upon hearing the label used by either the reliable or unreliable speaker (see Figure 19).

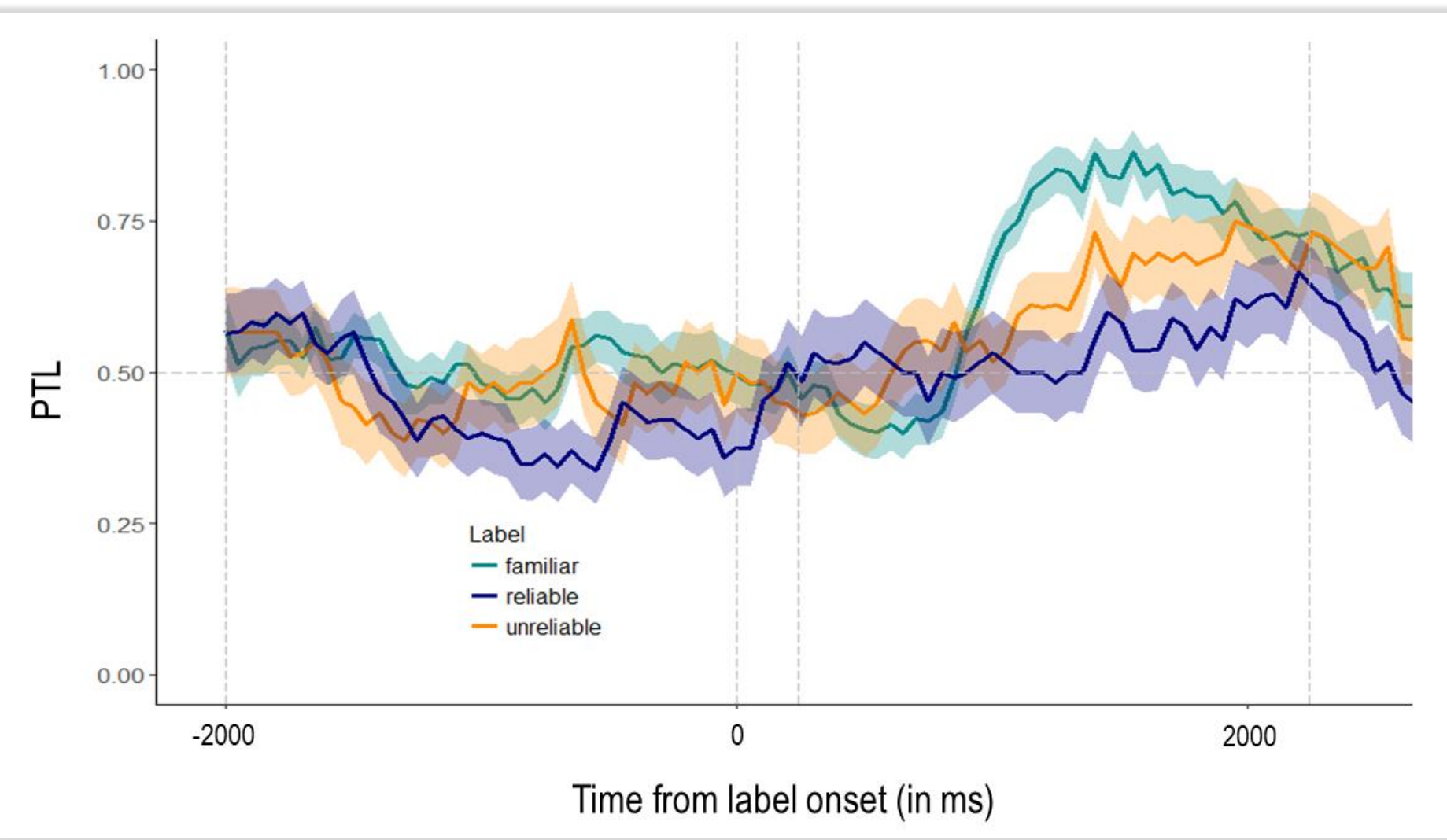

Figure 19. Time course of the proportion of target looking (PTL) in the Recognition task by condition (target object familiar, target labelled by reliable, target labelled by unreliable). PTL calculated as time spent looking at the target object divided by the time spent looking at target plus at any of the distractors during each $50 \mathrm{~ms}$ bin. Vertical dotted lines represent time windows used for time window analysis (pre-naming window: -2000 to $0 \mathrm{~ms}$ from label onset; post-naming window: 240 to $2240 \mathrm{~ms}$ ), horizontal dotted line represents chance level. 


\section{Interactive test phase}

I did not find any difference in explicit reliance on the novel label taught by the reliable or the unreliable source (see Figure 20). However, if we compare each condition to chance, infants were able to select the target object at rates above chance when the label was taught by a reliable source (binomial test, $\mathrm{p}=.045$ ).

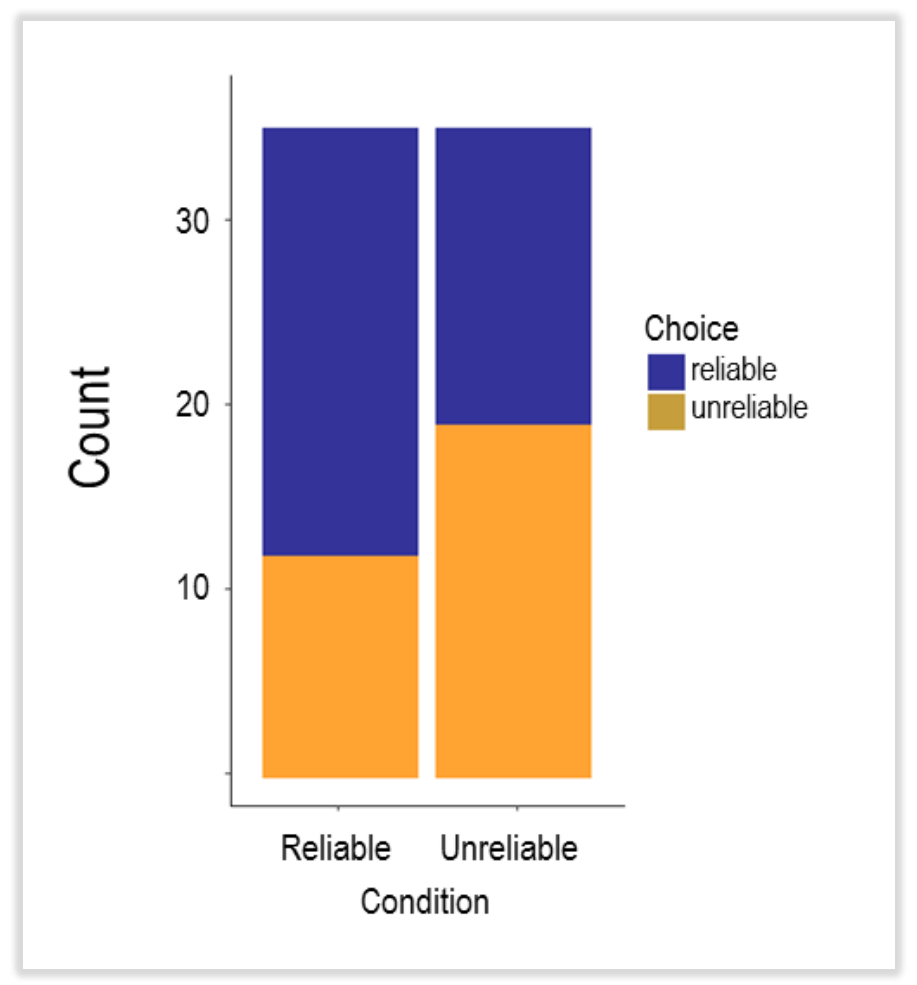

Figure 20. Infants' object choice in the interactive task by condition (target object labelled by reliable, target object labelled by unreliable). 


\subsubsection{Discussion}

In Study $2 b$, infants learned from both the previously reliable and the unreliable source. In the eye-tracking task, upon hearing the label used by the reliable informant, infants looked towards the object she had referred to with that label. But also in response to hearing the label used by the unreliable informant, infants looked towards the objects she had referred to with her label. I did not find any differences between infants' gaze behavior when relying on information initially provided by the reliable or by the unreliable source. Both time window analysis and the analysis of infants' looking behavior across the whole time course did not differ depending on the source of testimony.

The interactive task proved to be more difficult for the infants. I again did not find any differences between conditions, and infants' performance was at chance across the whole sample. Only if analyzing their response to each source individually, I find that their performance was above chance for the object labelled by the reliable informant. However, an interpretation of infants' performance in response to the reliable source individually is only informative to some degree, as there were no differences between conditions. Given that infants learned from either source enough to solve the eye-tracking task, I conclude that their performance in Study 2a was not due to confusion or high task demands. Rather it seems likely that infants rely on different strategies than preschoolers in their selective trust in testimony. Infants' performance across both studies $2 \mathrm{a}$ and $2 \mathrm{~b}$ suggests that infants likely do not attribute reliability to particular individuals. 


\section{General Discussion}

As a way to bypass the need for the many iterations of individual experiences required for individual learning, humans and several social non-human animal species have evolved a capacity to rely on information provided by others (Laland, 2004). Non-human animals learn from conspecifics through stimulus enhancement (Heyes, 1994; Nagell, Olguin, \& Tomasello, 1993) or emulation learning (Tomasello, 1990, 1996; Whiten et al., 2009). This means that they use the same tools a conspecific used to obtain the same goal, such as opening a nut with a rock, or that they try to replicate an outcome of a behavior. Human children on the other hand do not just focus on the outcome when learning from others. They pay a lot of attention to the process, draw inferences from ostensive cues, and try to replicate actions faithfully with a focus not just on what is demonstrated but also how and by whom (Csibra \& Gergely, 2009, 2011; Dean et al., 2014; Lyons et al., 2007). This high-fidelity in reliance on information from others gives rise to human cumulative culture and appears to be unique in the animal kingdom (Tennie et al., 2009; Tomasello, 2003). However, it can also lead to apparent biases, as can be seen in children's tendency to overimitate, that is to imitate parts of a demonstrated behavior, that is not instrumental in obtaining a certain result (Keupp et al., 2013; Nielsen, 2006). Here, children acquire artefacts that are not necessarily instrumental in obtaining a goal, but children's faithfulness in social information leads them to reproduce ambiguous or even questionable action demonstrations, likely due to normative or affiliative considerations (Ben Kenward, 2012; Keupp et 
al., 2013; Meltzoff, 2007; Nielsen \& Blank, 2011). In the domain of testimony, we similarly find apparent artefacts of children's credulity in reliance on socially conveyed information. Children will sometimes reproduce testimony by an adult, even if the usefullness of this testimony is questionable (e.g. Jaswal, 2010a; Jaswal et al., 2010; Vanderbilt et al., 2014). However, children are not always this credulous. Already infants can detect when somebody makes an error in their testimony and between the age of three to four, children can use this information to systematically prefer testimony from sources that do not make such errors (for reviews see Harris, 2012; Mills, 2013). In this thesis, I investigated the mechanisms behind preschoolers' selective trust in testimony and the early development of this ability.

\subsection{Selective Encoding of Testimony}

In my project, I first investigated children's selective trust in testimony when two sources of testimony with a history of accuracy or inaccuracy provide novel testimony that children seem to understand as contradictory, i.e. two different novel labels but for the same object (e.g. Koenig et al., 2004; Koenig \& Harris, 2005). In this case, children above three years of age consistently prefer testimony from the accurate source. When the 5-year-olds in my Study 1a were asked to identify the referent of the novel label that both sources used, they looked towards and selected the object labelled by the accurate source. Children's looking behavior correlated with their explicit identification of the referent in an interactive forced- 
choice task. This suggests children treat information by a reliable and an unreliable source differently, already affecting their spontaneous looking behavior. Further, 5-year-olds use these differences not just to adjust their reliance on testimony from the two acquainted sources, but also to adjust their reliance on additional testimony from a source with unknown reliability, as can be seen in the Contrast task in Study 1a. When a third person offered a novel label suggesting it referred to one of the two objects labelled by the differently reliable sources, children systematically discounted information from the unreliable source. This is again already visible in their looking behavior, which further suggests a stark difference in how testimony is treated depending on the source. But what are the underlying mechanisms of these differences? The findings of my first study are in line with a prominent assumption in the literature on selective trust, namely that differences in children's reactions towards reliable and unreliable sources manifest themselves at the stage of encoding.

According to the Blocking account, children may ignore any information that is provided by a source that has repeatedly been shown to provide inaccurate testimony (Kim et al., 2017; Mangardich \& Sabbagh, 2018). Children's preference for testimony from a reliable sources according to this account would thus be due to it being the only information they processed and learned.

Alternatively, children may attend to information by both reliable and unreliable sources, but block the processing of semantic information provided by someone who has been unreliable in the past. Such a Semantic gating account would assume 
a top-down gating mechanism that allows children to consider characteristics of the source during the encoding of testimony in order to guide their learning (Sabbagh et al., 2017; Sabbagh \& Shafman, 2009). This would in turn lead to children acquiring episodic information about the labelling event by either source, but no semantic information from testimony from an unreliable source. In my project, I investigated whether children generally differ in how they encode information from reliable and unreliable sources of testimony.

To this end, I confronted children with a reliable and an unreliable source who offered compatible novel testimony in Study 1b: two different novel labels for two different novel objects. Assuming that children generally encoded information differently depending on the source, I expected to find differences in how they attended to and learned from the testimony provided by either source. Following the assumption of the Blocking account, children should inhibit the acquisition of any further information from an unreliable source resulting in no evidence for any encoding of such information. Encoding of episodic information about the labelling event, as assumed by the Semantic gating account, however should at least let children draw the inference that the object in question had been labelled before. What both accounts have in common is that they assume that children generally learn less from an unreliable source.

In my Study $1 b$ however, I found that in fact children appear to learn from both reliable and unreliable sources to a very similar degree, given that both sources provided testimony compatible with each other. Children were able to identify an 
object that had been labelled by a reliable source among several distractors. They looked towards this object when they heared the respective label and were able to select the target object in an object choice task. In the same manner however, when asked to identify the referent of the novel label that the unreliable source used, children looked towards and selected the target object. Children's performance in the eye-tracking Recognition task suggests, that children did not fully block any information provided by an unreliable source - as the Blocking account hypothesizes. As already contingency learning is likely enough to solve a task like this (Bannard \& Tomasello, 2012), this does not necessarily suggest that children acquired semantic information from the testimony of the unreliable source. It does however suggest that children associated the label used by the unreliable source and the object she labelled. Further support for this observation comes from children's performance in the eye-tracking Disambiguation task. Children expected a novel label to refer to a novel object rather than to an object previously labelled by either the reliable or the unreliable informant. As this finding could result from children's acceptance of a novel name when they see an object that exemplifies a "nameless category" (Mervis \& Bertrand, 1994), children's performance in this task further excludes a Blocking account. If children inhibited any information from the unreliable source, they should treat both a completely novel object and an object labelled by an unreliable source as exemplifying a nameless category. In my Study $1 \mathrm{~b}$ however, children's assumption that the novel label refers to the completely novel object rather than the object labelled by the unreliable source suggests that they treat the later object not as nameless even 
though its label originated from a questionable source. While this shows that children did not fully disregard the testimony from the unreliable source, children's performance in the eye-tracking tasks could be explained by assuming differences only in semantic learning from the two sources. Episodic information about the labelling event of the unreliable source should suffices to pass both the eye-tracking Recognition and Disambiguation tasks. Children's performance in the interactive Recognition task however suggest that when accurate and inaccurate sources offer testimony that is not perceived to be in contradiction to each other, children will acquire semantic information from either source. When asked to identify the referent of the labelled provided by the reliable or the unreliable source, children had no problems in selecting the correct objects. This suggest that children do not generally encode information from a reliable and an unreliable source differently. Rather it seems that children in my Study $1 \mathrm{~b}$ learned from a previously inaccurate source in the same manner as from a previously accurate informant. This is similar to other studies that provided children with testimony from an unreliable source that was not contradicted by children's own knowledge or a more reliable source (Kim et al., 2017; Vanderbilt et al., 2014). As I did not find any differences in looking behavior across the whole time course of the experiment, it appears that children processed information by the two sources very similarly. Even the slightest doubt about the applicability of testimony by the unreliable source might have led to differences in the looking behavior, like a delayed response or prolonged exploration of distractors. However, we did not find evidence for any hesitation in response to hearing the label used by either 
source. Based on the findings of my first two studies, children's selective trust in testimony is likely not based on general differences in encoding of testimony by reliable and unreliable sources. It does not appear that children generally encode information in a different manner if it was provided by a source that proved to be inaccurate in prior interactions. Rather, children's selective social learning appears more nuanced than assumed by the Blocking or the Selective gating account. Perhaps these findings are not surprising, as children are willing social learners and tend to reproduce socially conveyed information even if the instrumental use of such information is questionable (see 1.1.3. Differences to human social learning). Children tend to rely on socially conveyed information with credulity, especially when such information is provided by adults and accompanied by ostensive cues. Apparently, this is also the case when the reliability of the source of such information is questionable.

\subsection{Default Trust or Rational Inferences?}

But does that mean that children default to trust in testimony, learning in the same manner from differently reliable sources and discount accuracy information just as long as the testimony is uncontested? The results of my studies could be a hint that a perceived contradiction in testimony from different sources triggers children's appreciation of accuracy information of those sources in order to resolve this conflict. If there is no contradiction that needs to be resolved, children might be biased to indiscriminately rely on any testimony without consideration of 
accuracy information, especially if this testimony is provided by an adult and with ostensive cues. Alternatively, children's reliance on uncontested testimony from unreliable sources could be caused by rational inferences at expression. Seen in this light, children's pragmatic strategy could be to rely on questionable testimony, in cases where it constitutes the only available information - even if children themselves are aware of it being questionable.

In order to conclusively investigate whether children retain accuracy information and can use it to adjust their reliance on testimony at expression even if testimony from the unreliable source was not contradicted at encoding, I used a between subject design were I provided children with either compatible or contradictory testimony from differently reliable sources. When the two sources provide contradictory information as in Study 1a, children discount information from the unreliable source in favor of a novel label by a third person when this novel label refers to either of the two objects labelled by the differently reliable sources (see Study 1a; Birch et al, 2008). If children - in case of uncontested testimonydefaulted to trust any source and discounted accuracy information, I expected them to show a different performance when the information provided by the two sources was not contradictory. Children should not discount information from the unreliable source in favor of the source with unknown reliability; rather they should show a performance at chance level. However, findings in my Study 1c suggest that when children hear an informant of unknown reliability refer to one of the two objects labelled by the differently reliable sources with a novel label, 
children infer that this novel label must refer to the object labelled by the inaccurate source. It did not make a difference for children, whether the accurate informant contradicted the information provided by the previously inaccurate informant or whether both provided compatible information. Already on their first trial in the Contrast task in Study 1c, children resolved the disambiguation by discounting testimony by the unreliable source in favor of novel testimony by a naïve source. For one, this shows that children's selectivity is not necessarily based on a contradiction in testimony by differently reliable sources when they acquire such information. Rather, children likely engage in rational inferences that lead them to rely even on testimony from the unreliable source, if it is the only available information and if there is thus no inducement to discount testimony from the unreliable source. However, in case novel testimony by a source with unknown reliability surfaces, children can use retained accuracy information, and reevaluate and adjust their reliance on testimony by unreliable sources between retrieval and reproduction. While these findings suggest, that children's selective trust in testimony can involve rational inferences at expression (Sobel \& Kushnir, 2013), there are several alternatives of what kind of inferences might enable children to selectively discard testimony from an unreliable source at expression.

Children might rely on metacognitive abilities, in that they represent their own knowledge or that of the informants as lacking precision or reliability (Heyes, 2016). This is in line for example with the findings from Koenig and colleagues (2004), who found that those children who were proficient in explicit metacognition were 
more likely to be selective. Children who were able to explicitly judged the reliability of an informant correctly were more likely to accept the reliable speaker's novel label for an unfamiliar object over the label provided by the inaccurate speaker (Koenig et al., 2004). Similarly, in my Study Set 1, most of the children were able to explicitly identify the more reliable informant in the manipulation check at the end of these studies, i.e. they were able to engage in explicit metacognitive assessment of the informants at the conclusion of the experiment.

Interestingly, I did not find any difference in certainty about the precision of their own recently acquired knowledge in form of the novel labels taught by either informant in Study 1b. Children did not appear uncertain about whether the label used by the unreliable informant would actually refer to the object she labelled. When they heard that label, their looking behavior towards the target object was not different from their looking behavior when hearing the label used by the reliable informant. Differences in metacognitive appraisal of own knowledge (i.e. certainty about the precision of what they just learned) would have likely lead to differences in the looking behavior, even if it only involved implicit metacognition (Darnell, 2016; Paulus, Proust, \& Sodian, 2013).

Other researches assume that children's selective trust decisions involve trait ascriptions. This assumption is supported by findings that children chose models selectively in accordance with traits corresponding to a task at hand, preferring a strong model for strength-related tasks and preferring a knowledgeable model for 
knowledge-related tasks (Hermes, Behne, Bich, et al., 2018; Hermes et al., 2015). This pattern of selective model choice held only for those children who were able to explicitly identify the attributes of both models at the conclusion of the experiment, suggesting that successful trait ascription plays an important role in selective trust (Hermes et al., 2015). This might suggest that children infer and ascribe specific competencies and traits to sources of testimony, and adjust their reliance at expression based on those inferences (Hermes, Behne, \& Rakoczy, 2018).

Alternatively, children might understand reliability not as a trait, but rather as a mental state of a person. Several studies found children's mental state ascription to correlate with their selective social learning (Brosseau-Liard, Penney, \& PoulinDubois, 2015; Crivello, Phillips, \& Poulin-Dubois, 2018). Those children who show proficiency in theory of mind abilities perform better on selective word learning tasks when the informants differ in epistemic knowledge, but not when they differed in non-epistemic abilities, such as strength (Brosseau-Liard et al., 2015). This might suggest that inferences about the reliability of individuals constitute inferences about the mental states of who is informative (Crivello et al., 2018).

In sum, children rely on socially conveyed information with credulity. This can be seen in their faithful reproduction of even irrelevant steps of an action demonstration when overimitating (e.g. Kenward, 2012; Keupp et al., 2013; Nielsen, 2006). Especially if such social information is provided with ostensive cues that children use to infer the generalizability of such information (Csibra \& Gergely, 2009, 2011). This credulity might also bias children to accept testimony from a 
source with questionably reliability, especially when there was no contradictory testimony by a more reliable source. In my studies however, I find that children are not necessarily mindless, gullible reproducers of social information. Rather it seems that even when they rely on testimony from an unreliable source with apparent credulity, even to a similar degree as on testimony from a reliable source, children still retain information about the (in-)accuracy of such testimony and are able to reevaluate their reliance between retrieval and reproduction of such testimony. My project thus shows that children can use more nuanced strategies to achieve selectivity in their trust in testimony than assumed by either the Blocking or the Semantic gating account in form of rational inferences at expression.

\subsection{On the Role of Contradictory Testimony}

Rather than generally encoding information differently depending of its source, children likely engage in rational inferences to decide whether or not to rely on testimony. When testimony from an unreliable sources was not contradicted by own knowledge or testimony from a more reliable source, children will learn from that testimony. However, they still retain the notion that this testimony is questionable and can engage in rational inferences at expression to discount unreliable testimony in favor of novel testimony. 
However, we cannot exclude that children rely on different strategies under different circumstances. Encoding and retaining novel information from two distinct sources as well as information about the accuracy of those sources and assessing all this information in inferences at expression seems considerably demanding on the cognitive abilities of children. In cases where two differently reliable sources offer conflicting testimony, children might thus resort to less demanding strategies. Children might perceive the contradiction in testimony as inducement that only testimony of one of the two sources is applicable, and thus resort to discount testimony by the unreliable source already at encoding. This strategy seems especially viable in cases where the reliability of the sources is apparent during encoding of information. While children need to rely on inferences about the reliability of the source if they only have information about the accuracy from prior interactions, it might be possible to circumvent such inferences when there are obvious cues for the sources accuracy. These cues can for example constitute categorical information about the source, such as their age (Jaswal \& Neely, 2006) or familiarity (Corriveau \& Harris, 2009a). But even behavioral cues are sometimes obvious during the acquisition of information and thus could allow children to resort to discounting information from one source at encoding in case of conflicting testimony instead of drawing inferences at expression. In the study by Sabbagh and Shafman (2009), a knowledgeable and an ignorant source provided conflicting testimony. The knowledgeability and ignorance here were introduced by the sources claiming to possess knowledge or claiming ignorance right when teaching the children novel labels. Thus, the 
reliability of the source was apparent to the children when they acquired information. The lack of semantic encoding from the self-proclaimed ignorant source might be a result of the particular circumstances, i.e. obvious unreliability while deciding whether to encode information and testimony from a more reliable source that can be perceived as better alternative. In my project, I found however that children do not generally encode information from distinct sources differently. Under circumstances where a reliable and an unreliable source provide testimony that is not conflicting with each other, children retain accuracy information and can use it to adjust their reliance later, even if they appear to encode information from the unreliable source in the same way as information from the reliable source.

As children do not necessarily need to represent properties of the cognitive processes of the agents in case their unreliability is apparent, the underlying mechanisms of selective trust under these circumstances could constitute associative processes (Heyes, 2017). For future research, it might be interesting to investigate whether children use different strategies depending on if the reliability of the sources is apparent when they provide novel information or if it has to be inferred.

\subsection{Early Development of Selective Trust in Testimony}

As I found no differences in learning from reliable and unreliable sources in case of uncontested testimony, selective trust in testimony in children at five of age is likely not based on general differences in encoding. The fact that children can still 
use inaccuracy information to adjust their reliance on testimony from unreliable source even if they appeared to learn this information to the same degree as from a reliable source, suggest the use of rational inferences at expression. These inferences likely involve abilities that young infants have not yet fully developed, such as metacognitive abilities, trait ascription or mental state ascription (Beran, Perner, \& Proust, 2012; Sodian, 2011; Yuill \& Pearson, 1998). Does that mean that infants rely on different strategies than preschoolers in their selective trust in testimony? To further explore the early development of trust in testimony, I investigated 2-year-olds response to testimony from two distinct sources with a history of accuracy or inaccuracy.

Previous studies that investigated the early development of selective trust in testimony typically provided infant below the age of three with only one source of testimony. This makes it difficult to discern an attribution of reliability to particular individuals -be it in form of trait or mental state ascription or a metacognitive appraisal of an informant's knowledge- from reduced learning due to situational variables. As infants are used to ostensive cues as markers of situations where typically generalizable information is conveyed (Csibra \& Gergely, 2009, 2011), inaccurate testimony presented with such cues might confuse them. This confusion could in turn lead to reduced learning without an appreciation of the particular inaccurate individual. Research with non-human animals suggests that selectivity in social learning is possible without an appreciation of particular individuals. 
Animals likely track the predictive relationship between events and if the situation is predictive of reduced success, they selectively learn less (Heyes \& Pearce, 2015).

To investigate whether infants like preschoolers attribute reliability to particular individuals, I provided 2-year-olds in my project with two sources of testimony that differed in prior accuracy. If infants attributed reliability to those sources like preschoolers and adjusted their reliance accordingly, they should selectively prefer testimony from the more accurate source. I found that unlike the 5-year-olds in Study 1a, infants at two years of age in my Study 2 a did not differ in their endorsement of novel labels based on prior reliability of the source. When hearing the label used by either informant, infants did not look preferably to either object and their object choice in the interactive task was similarly at chance. Further, infants did not expect a novel label provided by an unknown source to refer to the object labelled by the unreliable informant as preschoolers did; infants' performance in both the eye-tracking and interactive Contrast task in Study 2a was at chance. This might suggest that infants at two years of age do not yet attribute reliability to particular informants. However, it is also possible that the cognitive load required to solve this task was too high. In order to selectively reproduce information from a reliable source and to discount information from the unreliable source in favor of new information from a source of unknown reliability, infants would need to retain in memory both accuracy information about either source as well as testimony from either source. Failure in this task might thus not due to the lack of attribution of reliability, but due to constraints on memory in infants. 
Alternatively, the lack of differentiation between the two sources might be caused by confusion due to inaccurate labelling. If infants show reduced learning from the inaccurate source in single informant paradigms because of the presence of unusual cues, they should also show reduced learning even if an additional source did not provide such unusual cues. In order to exclude these alternative explanations and to investigate whether infants indeed do not attribute reliability to particular informants, I conducted a follow up study were I provided infants with compatible testimony from an accurate and an inaccurate source. If infants' performance in the first study was based on high task demands or confusion, they should show a performance no different from chance in Study $2 b$ as well. However, when they provided compatible testimony, infants learned from both the previously accurate as well as the inaccurate source. In an eye-tracking task, upon hearing the label used by the reliable informant, infants looked towards the object she had referred to with that label. But also in response to hearing the label used by the unreliable informant, infants looked towards the objects she had referred to with her novel label. I did not find any differences between infants' looking behavior when responding to information provided by either source across the whole time course of this study. Infants were not confused by the presence of an inaccurate labeler in the Study $2 b$ and apparently memorizing two labels for two novel objects did not exceed their cognitive load. This suggests that infants' failure to differentiate between the two sources in Study 2a was likely based on a lack of attribution of reliability to the individual sources. 
Assuming that 5-year-olds rely on rational inferences to adjust their reliance on testimony depending on source accuracy, the nature of these inferences might be a clue as to why infants are not yet able to attribute reliability to particular informants. As I laid out above, these inferences likely rely on metacognitive abilities or the ascription of traits or mental states to individuals. These are abilities that infants at 2 year of age have not fully developed yet (Beran et al., 2012; Sodian, 2011). Failure of infants selective trust decisions when confronted with two distinct sources of testimony might thus be due to developmental differences in representational abilities needed for rational inferences about sources' reliability (see also Sobel \& Kushnir, 2013).

But how do infants appear to learn less from a previously inaccurate source in other studies? Based on my findings, it is likely that they rely on different mechanisms than preschoolers. One possibility could be that they rely on processes that lead to them learning less when the circumstances are predictive of reduced usefulness of the transmitted information, similar to how non-human animals are assumed to be selective in their reliance on information derived from other (Heyes, 2017).

An alternative explanation for infant's selectivity could be derived from the assumption that older children's selective trust in testimony involves metacognitive abilities or mental state ascription. In this domain, infants (and nonhuman animals) show some proficiency without yet having acquired a full-blown metacognitive theory of mind (Call \& Tomasello, 2008). Instead, infants likely rely 
on tracking mechanisms to be able to follow other's perceptions, knowledge states and belief despite their limited cognitive resources (Butterfill \& Apperly, 2013). Tracking means encountering and registration of other's perception, knowledge states or beliefs without the involvement of explicit representations (Butterfill \& Apperly, 2013). Such a mechanism can explain infants' performance without assuming higher-order cognition like metacognitive abilities. Similarly, nonhuman animals appreciation of others perception and intention are assumed to rely on tracking mechanisms. For example, Call and Tomasello (2005) suggested that chimpanzees track the 'likely target' of others' visual access and understand something about its effects on behavior. By keeping track of other's perceptional or mental states without involving explicit representations of such states, infants and non-human animals achieve success in tasks that are typically assume to involve metacognitive abilities. A similar mechanism might enable infants (and non-human animals for that matter) to learn less from sources of testimony that provided cues of unreliability without a representation of the reliability of the particular individual. For example, the dogs in the study by Takaoka and colleagues (2015) might have tracked the inaccuracy of the cues provided by the human experimenter, which in turn led to reduced reliance on cues by this source. When subsequently a new informant with unknown reliability provided similar cues, dog's reliance on these cues increased back to a baseline level. This however does not necessarily require an ascription of reliability to the particular individuals providing testimony, rather with the introduction of a new, unknown informant, dog's tracking of the accuracy of the source's cue might have been interrupted and 
thus their reliance on socially conveyed cues reverted to default. Such a tracking mechanisms might also explain infants' failure in my studies to be selective when two informants provide conflicting testimony. Infants are known to have issues with tracking several distinct sources at once, especially when those sources are very similar to each other (Lindsay, Johnson, \& Kwon, 1991).

\subsection{Future Directions}

My project can tell us more about how children are sometimes selective in their trust in testimony and sometimes rely on testimony even from unreliable sources with credulity. For future research, it remains to investigate further why they are selective or rather why are they not always selective. The credulity with which children sometimes rely on testimony from questionable sources in case of uncontested testimony is reminiscent of overimitation. When imitating an adult's action demonstrations, children faithfully imitate even non-task-relevant actions (Nielsen, 2006). Overimitiation is assumed to be due to normative considerations of the actor demonstrating actions that are the culturally appropriate way of performing these actions. Children show evidence of this understanding as they protest if a third party omits those actions (Kenward, 2012; Keupp et al., 2013). But would they also protest if the person who demonstrates was inaccurate in the past? After all, this person has already shown that she does not conform to the conventions all the time. Furthermore, studying overimitation of actions demonstrated by accurate and inaccurate sources could tell us whether there is a 
difference in perceived normative force between the two sources that exceeds the domain of testimony. Altinok, Hernik, Kiraly, and Gergely (2018) found that children overimitate the speaker of a foreign language until subsequently a speaker of the children's native language demonstrated only task relevant actions. On the other hand, children did not cease to produce task-irrelevant actions when they had been demonstrated by a native speaker, even when subsequently a foreign speaker demonstrated only task relevant actions. Apparently, the native speaker's action demonstrations had a higher normative force. It seems plausible that similarly a previously accurate informant's action demonstrations would have a higher normative force compared to an inaccurate informant. Investigating the normative roots of selective social learning could help us understand why children who are prolific social learners at the same time sometimes appear very credulous.

\section{Conclusion}

In conclusion, children are willing social learners, especially if information is conveyed in an ostensive manner. This is unsurprising, as they need to learn a lot from others. However, this high fidelity sometimes leads to biased reliance on questionable information. Children's credulity in reliance on social information seems to override their ability to selectively disregard testimony by unreliable sources. So are children only able to rely on the ability to estimate sources reliability when deciding between two sources, and if an unreliable source provides uncontested testimony, children's credulity will lead to acceptance of 
questionable information? In my project, I found that even under circumstance where children appear to rely on unreliable sources to the same degree as reliable sources, they do not simply discount accuracy info. Rather, they retain information about the accuracy of such testimony and are able to adjust their reliance when additional information surfaces. Children do not generally encode information from reliable and unreliable sources differently, but they are also not gullible. As infants are not yet able to entertain such possibly metacognitive information about testimony, they likely rely on less demanding strategies, such as tracking, to adjust their reliance on testimony from others. My project could show that children are proficient in their reliance on testimony from others. They reproduce it with high fidelity, but at the same time, they are not as gullible as they sometimes appear to be. Rather, at least at five years of age, human children make rational inferences about the reliability of sources. Even if they initially rely on questionable testimony with credulity, these inferences let children adjust their reliance before deciding whether to reproduce such testimony. Children's trust in testimony is the result of opposing forces in form of attribution of reliability to particular sources of testimony and a credulity in reliance on socially conveyed information. 


\section{References}

Akhtar, N., \& Tomasello, M. (2000). The Social Nature of Words and Word learning. In R. Golinkoff, \& K. Hirsh-Pasek (Eds.), Becoming a Word Learner: A Debate on Lexical Acquisition. Oxford, U.K.: Oxford University Press.

Altinok, N., Hernik, M., Kiraly, I., \& Gergely, G. (2018, January). Shared-language Cue in Modulating Epistemic Trust in 4-year-old Children: An Over-imitation Study. Paper presented at the Budapest CEU Conference on Child Development 2018, Budapest, Hungary.

Bandura, A. (1986). Social Foundations of Thought and Action: A Social Cognitive Theory. Englewood Cliffs, NJ: Prentice-Hall.

Bannard, C., \& Tomasello, M. (2012). Can We Dissociate Contingency Learning from Social Learning in Word Acquisition by 24-Month-Olds? PLoS ONE, 7(11), e49881. https://doi.org/10.1371/journal.pone.0049881

Beran, M. J., Perner, J., \& Proust, J. (2012). Foundations of Metacognition. Oxford, U.K.: Oxford University Press.

Birch, S. A. J., Akmal, N., \& Frampton, K. L. (2010). Two-year-olds are Vigilant of Others' Non-verbal Cues to Credibility. Developmental Science, 13(2), 363-369. https://doi.org/10.1111/j.1467-7687.2009.00906.x

Birch, S. A. J., Vauthier, S. A., \& Bloom, P. (2008). Three- and Four-year-olds Spontaneously Use Others' Past Performance to Guide Their Learning. Cognition, 107(3), 1018-1034. https://doi.org/10.1016/j.cognition.2007.12.008

Bloom, L. (1993). The Transition from Infancy to Language: Acquiring the Power of Expression. New York: Cambridge University Press. https://doi.org/10.1017/CBO9780511752797 
Bloom, P. (2000). How Children Learn the Meanings of Words. Cambridge, MA: MIT Press.

Boyd, R., Richerson, P. J., \& Henrich, J. (2011). The Cultural Niche: Why Social Learning is Essential for Human Adaptation. Proceedings of the National Academy of Sciences, 108, 10918-10925. https://doi.org/10.1073/pnas.1100290108

Boyd, R., \& Richerson, P. J. (1988). An Evolutionary Model of Social Learning: The Effects of Spatial and Temporal Variation. In T. Zentall \& B. G. Galef (Eds.), Social learning: A Psychological and Biological Approach. Hillsdale, NJ: Erlbaum

Boyd, R., \& Richerson, P. J. (2005). The Origin and Evolution of Cultures. Oxford: Oxford Univ. Press.

Brosseau-Liard, P., Penney, D., \& Poulin-Dubois, D. (2015). Theory of Mind Selectively Predicts Preschoolers' Knowledge-based Selective Word Learning. British Journal of Developmental Psychology, 33(4), 464-475. https://doi.org/10.1111/bjdp.12107

Butterfill, S. A., \& Apperly, I. A. (2013). How to Construct a Minimal Theory of Mind. Mind $\mathcal{E}$ Language, 28(5), 606-637. https:/ / doi.org/10.1111/mila.12036

Call, J., Carpenter, M., \& Tomasello, M. (2005). Copying Results and Copying Actions in the Process of Social Learning: Chimpanzees (Pan Troglodytes) and Human Children (Homo sapiens). Animal Cognition, 8(3), 151-163. https://doi.org/10.1007/s10071004-0237-8

Call, J., \& Tomasello, M. (2008). Does the Chimpanzee Have a Theory of Mind? 30 Years Later. Trends in Cognitive Sciences, 12(5), 187-192.

Chou, L.-S., \& Richerson, P. J. (1992). Multiple Models in Social Transmission of Food Selection by Norway Rats, Rattus Norvegicus. Animal Behaviour, 44, 337-343. https://doi.org/10.1016/0003-3472(92)90039-C 
Corriveau, K., \& Harris, P. L. (2009a). Choosing Your Informant: Weighing Familiarity and Recent Accuracy. Developmental Science, 12(3), 426-437. https://doi.org/10.1111/j.1467-7687.2008.00792.x

Corriveau, K., \& Harris, P. L. (2009b). Preschoolers Continue to Trust a More Accurate Informant 1 Week After Exposure to Accuracy Information. Developmental Science, 12(1), 188-193. https:/ / doi.org/10.1111/j.1467-7687.2008.00763.x

Coussi-Korbel, S., \& Fragaszy, D. M. (1995). On the Relation Between Social Dynamics and Social Learning. Animal Behaviour, 50(6), 1441-1453. https:// doi.org/10.1016/0003$3472(95) 80001-8$

Crivello, C., Phillips, S., \& Poulin-Dubois, D. (2018). Selective Social Learning in Infancy: Looking for Mechanisms. Developmental Science, 21(3), e12592. https://doi.org/10.1111/desc.12592

Csibra, G., \& Gergely, G. (2009). Natural Pedagogy. Trends in Cognitive Sciences, 13(4), 148153. https://doi.org/10.1016/j.tics.2009.01.005

Csibra, G., \& Gergely, G. (2011). Natural Pedagogy as Evolutionary Adaptation. Philosophical Transactions of the Royal Society B: Biological Sciences, 366(1567), 1149-1157. https://doi.org/10.1098/rstb.2010.0319

Darnell, C. A. (2016). Children's Understanding of Uncertainty (Doctoral dissertation). Retrieved from University of Birmingham Institutional Research Archive (ID Code 6562).

Dean, L. G., Vale, G. L., Laland, K. N., Flynn, E., \& Kendal, R. L. (2014). Human Cumulative Culture: A Comparative Perspective. Biological Reviews, 89(2), 284-301. https://doi.org/10.1111/brv.12053

Dink, J., \& Ferguson, B. (2018). EyetrackingR. R package version 0.1.7, http:/ / www.eyetracking-R.com 
DiYanni, C., Nini, D., Rheel, W., \& Livelli, A. (2012). ‘I Won't Trust You if I Think You're Trying to Deceive $\mathrm{Me}^{\prime \prime}$ : Relations Between Selective Trust, Theory of Mind, and Imitation in Early Childhood.' Journal of Cognition and Development, 13(3), 354-371. https:// doi.org/10.1080/15248372.2011.590462

Galef, B. G., \& Laland, K. N. (2005). Social Learning in Animals: Empirical Studies and Theoretical Models. BioScience, 55(6), 489. https://doi.org/10.1641/00063568(2005)055[0489:SLIAES]2.0.CO;2

Gariépy, J.-F., Watson, K. K., Du, E., Xie, D. L., Erb, J., Amasino, D., \& Platt, M. L. (2014). Social Learning in Humans and Other Animals. Frontiers in Neuroscience, 8. https://doi.org/10.3389/fnins.2014.00058

Harris, P. L. (2012). Trusting What You're Told: How Children Learn From Others. Cambridge, MA: Harvard University Press.

Harris, P. L., \& Koenig, M. A. (2006). Trust in Testimony: How Children Learn About Science and Religion. Child Development, 77(3), 505-524. https:// doi.org/10.1111/j.14678624.2006.00886.x

Harris, P. L., \& Lane, J. D. (2014). Infants Understand How Testimony Works. Topoi, 33(2), 443-458. https:/ / doi.org/10.1007/s11245-013-9180-0

Hermes, J., Behne, T., Bich, A. E., Thielert, C., \& Rakoczy, H. (2018). Children's Selective Trust Decisions: Rational Competence and Limiting Performance Factors. Developmental Science, 21(2), e12527. https:// doi.org/10.1111/desc.12527

Hermes, J., Behne, T., \& Rakoczy, H. (2015). The Role of Trait Reasoning in Young Children's Selective Trust. Developmental Psychology, 51(11), 1574-1587. https://doi.org/10.1037/dev0000042 
Hermes, J., Behne, T., \& Rakoczy, H. (2018). The Development of Selective Trust: Prospects for a Dual-Process Account. Child Development Perspectives, 12(2), 134-138. https://doi.org/10.1111/cdep.12274

Heyes, C. (1994). Social Learning in Animals: Categories and Mechanisms. Biological Reviews of the Cambridge Philosophical Society, 69(2), 207-231.

Heyes, C., \& Pearce, J. M. (2015). Not-so-social Learning Strategies. Proceedings of the Royal Society B: Biological Sciences, 282(1802), 20141709-20141709. https://doi.org/10.1098/rspb.2014.1709

Heyes, C. (2016). Who Knows? Metacognitive Social Learning Strategies. Trends in Cognitive Sciences, 20(3), 204-213. https:// doi.org/10.1016/j.tics.2015.12.007

Heyes, C. (2017). When Does Social Learning Become Cultural Learning? Developmental Science, 20(2), e12350. https:/ / doi.org/10.1111/desc.12350

Horner, V., \& Whiten, A. (2005). Causal Knowledge and Imitation/Emulation Switching in Chimpanzees (Pan troglodytes) and Children (Homo sapiens). Animal Cognition, 8(3), 164-181. https:/ / doi.org/10.1007/s10071-004-0239-6

Horst, J. S., \& Hout, M. C. (2016). The Novel Object and Unusual Name (NOUN) Database: A Collection of Novel Images for Use in Experimental Research. Behavior Research Methods, 48(4), 1393-1409. https:/ / doi.org/10.3758/s13428-015-0647-3

Humle, T., \& Matsuzawa, T. (2002). Ant-dipping Among the Chimpanzees of Bossou, Guinea, and Some Comparisons With Other Sites. American Journal of Primatology, 58(3), 133-148. https://doi.org/10.1002/ajp.10055

Jaswal, V. K. (2010a). Believing What you're Told: Young Children's Trust in Unexpected Testimony About the Physical World. Cognitive Psychology, 61(3), 248-272. https:// doi.org/10.1016/j.cogpsych.2010.06.002 
Jaswal, V. K. (2010b). Explaining the Disambiguation Effect: Don't Exclude Mutual Exclusivity. Journal of Child Language, 37(01), 95. https://doi.org/10.1017/S0305000909009519

Jaswal, V. K., Croft, A. C., Setia, A. R., \& Cole, C. A. (2010). Young Children Have a Specific, Highly Robust Bias to Trust Testimony. Psychological Science, 21(10), 1541-1547. https://doi.org/10.1177/0956797610383438

Jaswal, V. K., \& Neely, L. A. (2006). Adults Don't Always Know Best: Preschoolers Use Past Reliability Over Age When Learning New Words. Psychological Science, 17(9), 757-758. https://doi.org/10.1111/j.1467-9280.2006.01778.x

Kamin, L.J. (1969). Selective Association and Conditioning. In N. J. Mackintosh \& F. W. K. Honig (Eds.), Fundamental Issues in Associative Learning. Halifax: Dalhousie University Press.

Kenward, B., Karlsson, M., \& Persson, J. (2011). Over-imitation is Better Explained by Norm Learning than by Distorted Causal Learning. Proceedings of the Royal Society B: Biological Sciences, 278(1709), 1239-1246. https://doi.org/10.1098/rspb.2010.1399

Kenward, B. (2012). Over-imitating Preschoolers Believe Unnecessary Actions are Normative and Enforce their Performance by a Third Party. Journal of Experimental Child Psychology, 112(2), 195-207. https:/ / doi.org/10.1016/j.jecp.2012.02.006

Keupp, S., Behne, T., \& Rakoczy, H. (2013). Why do Children Overimitate? Normativity is Crucial. Journal of Experimental Child Psychology, 116(2), 392-406. https://doi.org/10.1016/j.jecp.2013.07.002

Keupp, S., Behne, T., Zachow, J., Kasbohm, A., \& Rakoczy, H. (2015). Over-imitation is not Automatic: Context Sensitivity in Children's Overimitation and Action Interpretation of Causally Irrelevant Actions. Journal of Experimental Child Psychology, 130, 163-175. https://doi.org/10.1016/j.jecp.2014.10.005 
Kim, S., Paulus, M., \& Kalish, C. (2017). Young Children's Reliance on Information From Inaccurate Informants. Cognitive Science, 41, 601-621. https://doi.org/10.1111/cogs.12471

Koenig, M. A., Clement, F., \& Harris, P. L. (2004). Trust in Testimony: Children's Use of True and False Statements. Psychological Science, 15(10), 694-698. https://doi.org/10.1111/j.0956-7976.2004.00742.x

Koenig, M. A., \& Harris, P. L. (2005). Preschoolers Mistrust Ignorant and Inaccurate Speakers. Child Development, 76(6), 1261-1277. https://doi.org/10.1111/j.14678624.2005.00849.x

Koenig, M. A., \& Echols, C. H. (2003). Infants' Understanding of False Labeling Events: The Referential Roles of Words and the Speakers who use them. Cognition, 87(3), 179-208.

Koenig, M. A., \& Harris, P. L. (2007). The Basis of Epistemic Trust: Reliable Testimony or Reliable Sources? Episteme, 4(03), 264-284. https://doi.org/10.3366/E1742360007000081

Koenig, M. A., \& Woodward, A. L. (2010). Sensitivity of 24-month-olds to the Prior Inaccuracy of the Source: Possible Mechanisms. Developmental Psychology, 46(4), 815826. https://doi.org/10.1037/a0019664

Krogh-Jespersen, S., \& Echols, C. H. (2012). The Influence of Speaker Reliability on First Versus Second Label Learning: Speaker Reliability and Word Learning. Child Development, no-no. https:// doi.org/10.1111/j.1467-8624.2011.01713.x

Laland, K. N., \& Williams, K. (1997). Shoaling Generates Social Learning of Foraging Information in Guppies. Animal Behaviour, 53(6), 1161-1169.

Laland, K. N. (2004). Social Learning Strategies. Animal Learning E Behavior, 32(1), 4-14. https://doi.org/10.3758/BF03196002 
Li, X., \& Yow, W. Q. (2018). Willingness to Revise own Testimony: 3- and 4-year-olds' Selective Trust in Unexpected Testimony From Accurate and Inaccurate informants. Journal of Experimental Child Psychology, 173, 1-15. https:// doi.org/10.1016/j.jecp.2018.03.008

Lindsay, D. S., Johnson, M. K., \& Kwon, P. (1991). Developmental Changes in Memory Source Monitoring. Journal of Experimental Child Psychology, 52(3), 297-318.

Lyons, D. E., Young, A. G., \& Keil, F. C. (2007). The Hidden Structure of Overimitation. Proceedings of the National Academy of Sciences, 104(50), 19751-19756. https://doi.org/10.1073/pnas.0704452104

Mangardich, H., \& Sabbagh, M. A. (2018). Children Remember Words from Ignorant Speakers but do not Attach Meaning: Evidence from Event-related Potentials. Developmental Science, 21(2), e12544. https:// doi.org/10.1111/desc.12544

Markman, E. M., \& Wachtel, G. F. (1988). Children's Use of Mutual Exclusivity to Constrain the Meanings of Words. Cognitive Psychology, 20(2), 121-157. https://doi.org/10.1016/0010-0285(88)90017-5

Mascaro, O., \& Sperber, D. (2009). The Moral, Epistemic, and Mindreading Components of Children's Vigilance Towards Deception. Cognition, 112(3), 367-380. https://doi.org/10.1016/j.cognition.2009.05.012

Mason, J. R., \& Reidinger, R. F. (1982). Observational Learning of Food Aversions in Redwinged Blackbirds (Agelaius phoeniceus). The Auk, 99(3), 548-554.

Meltzoff, A. N. (2007). 'Like me': A Foundation for Social Cognition. Developmental Science, 10(1), 126-134. https:// doi.org/10.1111/j.1467-7687.2007.00574.x

Menzel, E. W. (1973). Chimpanzee Spatial Memory Organization. Science, 182(4115), 943-945. https://doi.org/10.1126/science.182.4115.943 
Menzel, E. W. (1974). A Group of Young Chimpanzees in a One-Acre Field. In A. M. Schrier \& F. Stollnitz (Eds.), Behavior of Nonhuman Primates (Vol. 5). New York: Academic Press. https:// doi.org/10.1016/B978-0-12-629105-6.50009-2

Merriman, W. E., \& Schuster, J. M. (1991). Young Children's Disambiguation of Object Name Reference. Child Development, 62(6), 1288-1301. https:/ / doi.org/10.1111/j.14678624.1991.tb01606.x

Mervis, C. B., \& Bertrand, J. (1994). Acquisition of the Novel Name-Nameless Category (N3C) Principle. Child Development, 65(6), 1646-1662. https://doi.org/10.1111/j.14678624.1994.tb00840.x

Mills, C. M. (2013). Knowing When to Doubt: Developing a Critical Stance when Learning from Others. Developmental Psychology, 49(3), 404-418. https://doi.org/10.1037/a0029500

Nagell, K., Olguin, R. S., \& Tomasello, M. (1993). Processes of Social Learning in the Tool Use of Chimpanzees (Pan troglodytes) and Human Children (Homo sapiens). Journal of Comparative Psychology, 107(2), 174-186.

Nielsen, M. (2006). Copying Actions and Copying Outcomes: Social Learning Through the Second Year. Developmental Psychology, 42(3), 555.

Nielsen, M., \& Blank, C. (2011). Imitation in Young Children: When Who Gets Copied is More Important Than What Gets Copied. Developmental Psychology, 47(4), 1050-1053. https://doi.org/10.1037/a0023866

Nielsen, M., Mushin, I., Tomaselli, K., \& Whiten, A. (2014). Where Culture Takes Hold: “Overimitation" and Its Flexible Deployment in Western, Aboriginal, and Bushmen Children. Child Development, 85(6), 2169-2184. https://doi.org/10.1111/cdev.12265 
Nielsen, M., Simcock, G., \& Jenkins, L. (2008). The Effect of Social Engagement on 24-montholds' Imitation from Live and Televised Models. Developmental Science, 11(5), 722-731. https://doi.org/10.1111/j.1467-7687.2008.00722.x

Nielsen, M., \& Tomaselli, K. (2010). Overimitation in Kalahari Bushman Children and the Origins of Human Cultural Cognition. Psychological Science, 21(5), 729-736. https://doi.org/10.1177/0956797610368808

Nurmsoo, E., \& Robinson, E. J. (2009). Children's Trust in Previously Inaccurate Informants Who Were Well or Poorly Informed: When Past Errors Can Be Excused. Child Development, 80(1), 23-27. https://doi.org/10.1111/j.1467-8624.2008.01243.x

Over, H., \& Carpenter, M. (2012). Putting the Social into Social Learning: Explaining both Selectivity and Fidelity in Children's Copying Behavior. Journal of Comparative Psychology, 126(2), 182-192. https://doi.org/10.1037/a0024555

Pasquini, E. S., Corriveau, K. H., Koenig, M., \& Harris, P. L. (2007). Preschoolers Monitor the Relative Accuracy of Informants. Developmental Psychology, 43(5), 1216-1226. https://doi.org/10.1037/0012-1649.43.5.1216

Paulus, M., Proust, J., \& Sodian, B. (2013). Examining Implicit Metacognition in 3.5-year-old Children: An Eye-tracking and Pupillometric Study. Frontiers in Psychology, 4, 145. https://doi.org/10.3389/fpsyg.2013.00145

Pea, R. D. (1982). Origins of Verbal Logic: Spontaneous Denials by Two- and Three-year Olds. Journal of Child Language, 9(03). https:// doi.org/10.1017/S0305000900004931

Pexman, P. M., \& Glenwright, M. (2007). How Do Typically Developing Children Grasp the Meaning of Verbal Irony? Journal of Neurolinguistics, 20(2), 178-196. https://doi.org/10.1016/j.jneuroling.2006.06.001

Richerson, P. J., \& Boyd, R. (2005). Not by Genes Alone: How Culture Transformed Human Evolution. Chicago, IL: University of Chicago Press. 
Sabbagh, M. A., \& Baldwin, D. A. (2001). Learning Words from Knowledgeable versus Ignorant Speakers: Links Between Preschoolers' Theory of Mind and Semantic Development. Child Development, 72(4), 1054-1070. https:// doi.org/10.1111/14678624.00334

Sabbagh, M. A., Koenig, M. A., \& Kuhlmeier, V. A. (2017). Conceptual Constraints and Mechanisms in Children's Selective Learning. Developmental Science, 20(2), e12415. https://doi.org/10.1111/desc.12415

Sabbagh, M. A., \& Shafman, D. (2009). How Children Block Learning From Ignorant Speakers. Cognition, 112(3), 415-422. https:// doi.org/10.1016/j.cognition.2009.06.005

Schmid, B., Karg, K., Perner, J., \& Tomasello, M. (2017). Great Apes are Sensitive to Prior Reliability of an Informant in a Gaze Following Task. PLOS ONE, 12(11), e0187451. https://doi.org/10.1371/journal.pone.0187451

Scofield, J., \& Behrend, D. A. (2008). Learning Words from Reliable and Unreliable Speakers. Cognitive Development, 23(2), 278-290. https:/ / doi.org/10.1016/j.cogdev.2008.01.003

Seyfarth, R. M., Cheney, D. L., \& Marler, P. (1980). Monkey Responses to Three Different Alarm Calls: Evidence of Predator Classification and Semantic Communication. Science, 210(4471), 801-803.

Sobel, D. M., \& Kushnir, T. (2013). Knowledge Matters: How Children Evaluate the Reliability of Testimony as a Process of Rational Inference. Psychological Review, 120(4), 779-797. https://doi.org/10.1037/a0034191

Sodian, B. (2011). Theory of Mind in Infancy: Theory of Mind in Infancy. Child Development Perspectives, 5(1), 39-43. https:// doi.org/10.1111/j.1750-8606.2010.00152.x

Takaoka, A., Maeda, T., Hori, Y., \& Fujita, K. (2015). Do Dogs Follow Behavioral Cues from an Unreliable Human? Animal Cognition, 18(2), 475-483. https://doi.org/10.1007/s10071-014-0816-2 
Templeton, J. J., \& Giraldeau, L.-A. (1996). Vicarious Sampling: the Use of Personal and Public Information by Starlings Foraging in a Simple Patchy Environment. Behavioral Ecology and Sociobiology, 38(2), 105-114. https://doi.org/10.1007/s002650050223

Tennie, C., Call, J., \& Tomasello, M. (2009). Ratcheting up the Ratchet: on the Evolution of Cumulative Culture. Philosophical Transactions of the Royal Society B: Biological Sciences, 364(1528), 2405-2415. https://doi.org/10.1098/rstb.2009.0052

Tolman, E. C., \& Honzik, C. H. (1930). Introduction and Removal of Reward, and Maze Performance in Rats. University of California Publications in Psychology, 4, 257-275. Tomasello, M. (1990). Cultural Transmission in the Tool Use and Communicatory Signaling of Chimpanzees? In S. T. Parker \& K. R. Gibson (Eds.), "Language" and Intelligence in Monkeys and Apes (pp. 274-311). Cambridge, MA: Cambridge University Press. https://doi.org/10.1017/CBO9780511665486.012

Tomasello, M. (1996). Do Apes Ape? In C. M. Heyes \& B. G. Galef, Jr. (Eds.), Social Learning in Animals: The Roots of Culture (pp. 319-346). London, U.K.: Academic Press. https://doi.org/10.1016/B978-012273965-1/50016-9

Tomasello, M. (2003). The Cultural Origins of Human Cognition (4. print). Cambridge, MA: Harvard Univ. Press.

van Bergen, Y., Coolen, I., \& Laland, K. N. (2004). Nine-spined Sticklebacks Exploit the Most Reliable Source when Public and Private Information Conflict. Proceedings of the Royal Society B: Biological Sciences, 271(1542), 957-962.

https://doi.org/10.1098/rspb.2004.2684

Vanderbilt, K. E., Heyman, G. D., \& Liu, D. (2014). In the Absence of Conflicting Testimony Young Children Trust Inaccurate Informants. Developmental Science, 17(3), 443-451. https://doi.org/10.1111/desc.12134 
Vanderbilt, K. E., Liu, D., \& Heyman, G. D. (2011). The Development of Distrust. Child Development, 82(5), 1372-1380. https://doi.org/10.1111/j.1467-8624.2011.01629.x von Holzen, K., \& Mani, N. (2012). Language Nonselective Lexical Access in Bilingual Toddlers. Journal of Experimental Child Psychology, 113(4), 569-586.

https://doi.org/10.1016/j.jecp.2012.08.001

Vosniadou, S., \& Brewer, W. F. (1987). Theories of Knowledge Restructuring in Development. Review of Educational Research, 57(1), 51-67. https://doi.org/10.3102/00346543057001051

Vygotsky, L. S. (1980). Mind in Society: The Development of Higher Psychological Processes. Cambridge, MA: Harvard University Press.

Vygotsky, L. S. (1997). The Collected Works of L. S. Vygotsky. Vol.4. The History of the Development of Higher Mental Functions. New York, NY: Plenum.

Whiten, A., Goodall, J., McGrew, W. C., Nishida, T., Reynolds, V., Sugiyama Y., Tutin, C. E. G., Wrangham, R. W., \& Boesch, C. (2001). Charting Cultural Variation in Chimpanzees. Behaviour, 138(11-12), 1481-1516. https://doi.org/10.1163/156853901317367717

Whiten, A., McGuigan, N., Marshall-Pescini, S., \& Hopper, L. M. (2009). Emulation, Imitation, Over-imitation and the Scope of Culture for Child and Chimpanzee. Philosophical Transactions of the Royal Society B: Biological Sciences, 364(1528), 2417-2428. https:// doi.org/10.1098/rstb.2009.0069

Wilkinson, G. S. (1992). Information Transfer at Evening Bat Colonies. Animal Behaviour, 44, 501-518. https:/ / doi.org/10.1016/0003-3472(92)90059-I

Woodward, A. L., \& Markman, E. M. (1998). Early Word Learning. In W. Damon, D. Kuh, \& R. Siegler (Eds.), Handbook of Child Psychology: Volume 2: Cognition, Perception, and Language. (pp. 371-420). Hoboken, NJ: John Wiley \& Sons Inc. 
Yuill, N., \& Pearson, A. (1998). The Development of Bases for Trait Attribution: Children's Understanding of Traits as Causal Mechanisms Based on Desire. Developmental Psychology, 34(3), 574-586. https:// doi.org/10.1037/0012-1649.34.3.574 